\title{
ON THE IRREDUCIBLE COMPONENTS OF SOME CRYSTALLINE DEFORMATION RINGS
}

\author{
ROBIN BARTLETT \\ Max Planck Institute for Mathematics, Germany; \\ email: robinbartlett18@mpim-bonn.mpg.de
}

Received 12 January 2020; accepted 15 February 2020

\begin{abstract}
We adapt a technique of Kisin to construct and study crystalline deformation rings of $G_{K}$ for a finite extension $K / \mathbb{Q}_{p}$. This is done by considering a moduli space of Breuil-Kisin modules, satisfying an additional Galois condition, over the unrestricted deformation ring. For $K$ unramified over $\mathbb{Q}_{p}$ and Hodge-Tate weights in $[0, p]$, we study the geometry of this space. As a consequence, we prove that, under a mild cyclotomic-freeness assumption, all crystalline representations of an unramified extension of $\mathbb{Q}_{p}$, with Hodge-Tate weights in $[0, p]$, are potentially diagonalizable.
\end{abstract}

2010 Mathematics Subject Classification: 11F80 (primary); 11F33 (secondary)

\section{Introduction}

1.1. Motivation and results. In this paper, we establish new instances of potential diagonalizability, a notion introduced in [1], which concerns the geometry of crystalline deformation rings. If $K / \mathbb{Q}_{p}$ is a finite extension, then a $p$-adic representation of $G_{K}=\operatorname{Gal}(\bar{K} / K)$ is potentially diagonalizable if, after possibly restricting to a finite extension of $K$, it lies on the same irreducible component of an appropriate crystalline deformation ring as a direct sum of characters. The significance of this definition comes from one of the main results of [1], which establishes modularity lifting theorems for conjugate self-dual representations of a $\mathrm{CM}$ number field $F$, which are potentially diagonalizable at places of $F$ dividing $p$.

It is currently unclear whether or not all potentially crystalline representations are expected to be potentially diagonalizable. While there are no known counterexamples, positive results have been established in only two situations:

(c) The Author 2020. This is an Open Access article, distributed under the terms of the Creative Commons Attribution licence (http://creativecommons.org/licenses/by/4.0/), which permits unrestricted re-use, distribution, and reproduction in any medium, provided the original work is properly cited. 
(1) When $K / \mathbb{Q}_{p}$ is unramified and the representation is crystalline with Hodge-Tate weights contained in the interval $[0, p-1]$. See the work of Gao-Liu [13].

(2) When $K / \mathbb{Q}_{p}$ is arbitrary and the representation is two-dimensional and potentially Barsotti-Tate (that is, potentially crystalline with Hodge-Tate weights $\{0,1\})$. See the work of Kisin and Gee [14, 22] as well as [15, 3.4.1].

The method used to establish (1) proceeds as follows. First, one replaces $K$ by a sufficiently large unramified extension so that the reduction modulo $p$ of the representation in question is a successive extension of characters. One then uses Fontaine-Laffaille theory to construct a congruence to a representation which has the same Hodge-Tate weights and which is itself a successive extension of crystalline characters. Due to $K / \mathbb{Q}_{p}$ being unramified and the Hodge-Tate weights being assumed to lie in $[0, p-1]$, the relevant deformation rings consist of a single irreducible component. From this, it is easy to deduce potential diagonalizability (one has to show that the successive extension of characters can be replaced by a direct sum, which can be done in complete generality).

If $K / \mathbb{Q}_{p}$ ramifies, or the Hodge-Tate weights lie outside [0,p-1], this approach does not work; the relevant deformation rings rarely consist of a single component and Fontaine-Laffaille theory is unavailable. Instead, (2) is proven by constructing, for $R$ an appropriate Barsotti-Tate deformation ring, a moduli space of Breuil-Kisin modules $\mathcal{L}$ over $\operatorname{Spec} R$. The morphism $\mathcal{L} \rightarrow \operatorname{Spec} R$ becomes an isomorphism after inverting $p$; this reduces the problem of understanding the components of $\operatorname{Spec} R$ to that of understanding the components of $\mathcal{L}$. By sufficiently controlling the geometry of $\mathcal{L}$, this is further reduced to understanding the components of the fibre of $\mathcal{L} \rightarrow$ Spec $R$ over the closed point, which can be done via explicit calculations.

In this paper, we explore the extent with which this second strategy can be adapted beyond the Barsotti-Tate situation. In doing so, we obtain new results towards potential diagonalizability when $K / \mathbb{Q}_{p}$ is unramified and the Hodge-Tate weights are contained in the interval $[0, p]$.

THEOREM 1.1.1. Suppose $K / \mathbb{Q}_{p}$ is unramified and $V$ is a crystalline representation of $G_{K}$ on a finite-dimensional $\overline{\mathbb{Q}}_{p}$-vector space, with HodgeTate weights contained in $[0, p]$. Assume the mod $p$ semisimplification $\bar{V}$ of $V$ is strongly cyclotomic-free, in the sense of Definition 4.3.7. Then $V$ is potentially diagonalizable.

We also expect our approach will be useful in more general situations (indeed, since writing this paper, we have adapted the techniques here to apply in ramified situations; see [4]). 
1.2. Method. The starting point of this paper is the results of Gee-Liu-Savitt [16]. They consider, for $K / \mathbb{Q}_{p}$ unramified, a lattice $\rho: G_{K} \rightarrow \mathrm{GL}_{2}\left(\overline{\mathbb{Z}}_{p}\right)$ inside a crystalline representation with Hodge-Tate weights in $[0, p]$. They show that if $\bar{\rho}=\rho \otimes_{\overline{\mathbb{Z}}_{p}} \overline{\mathbb{F}}_{p}$ is reducible, then there exists $\rho^{\prime}: G_{K} \rightarrow \mathrm{GL}_{2}\left(\overline{\mathbb{Z}}_{p}\right)$ with the same Hodge-Tate weights as $\rho$, with $\rho^{\prime} \equiv \rho$ modulo $p$, and with $\rho^{\prime}$ an extension of two crystalline characters. The following are two key ingredients that go into their proof.

- If $\overline{\mathfrak{M}}$ is the reduction modulo $p$ of the Breuil-Kisin module associated with $\rho$, then they show that $\overline{\mathfrak{M}}$ is, what we shall call in this paper, strongly divisible. This means that there exists an $\mathbb{F}_{p}[[u]]$-basis $\left(e_{i}\right)$ of $\overline{\mathfrak{M}}$ and integers $r_{i} \in[0$, $p]$ such that $\left(u^{r_{i}} e_{i}\right)$ generate $\varphi(\overline{\mathfrak{M}})$ over $\mathbb{F}_{p}\left[\left[u^{p}\right]\right]$. These $r_{i}$ correspond to the Hodge-Tate weights of $\rho$.

- Since $\bar{\rho}$ is reducible, $\overline{\mathfrak{M}}$ is an extension between two rank-one strongly divisible Breuil-Kisin modules. Gee-Liu-Savitt compute the relevant extension group in the category of strongly divisible Breuil-Kisin modules, and show they have dimension (modulo an error term) less than or equal to the dimension of a space of crystalline extensions $H_{f}^{1}\left(G_{K},-\right)$.

The lift $\rho^{\prime}$ is produced by analysing the Breuil-Kisin modules associated with crystalline representations that are extensions of two characters, and showing that such an extension may be chosen so that the Breuil-Kisin module is congruent to $\overline{\mathfrak{M}}$ modulo $p$.

This goes part of the way to proving potential diagonalizability of $\rho$. However, it unclear whether or not $\rho$ and $\rho^{\prime}$ lie in the same irreducible component of a crystalline deformation ring. We address this by proving that two such crystalline representations $\rho$ and $\rho^{\prime}$, with Hodge-Tate weights contained in $[0, p]$, are contained in the same irreducible component of a crystalline deformation ring if the Breuil-Kisin modules associated with $\rho$ and $\rho^{\prime}$ are congruent modulo $p$. This is deduced from the following theorem, which summarizes the key new results in this paper.

THEOREM 1.2.1. Let $\mathbb{F}$ be a finite field of characteristic $p$ and $V_{\mathbb{F}}$ a continuous representation of $G_{K}$ on a finite-dimensional $\mathbb{F}$-vector space. Let $R$ be the unrestricted framed deformation ring of $V_{\mathbb{F}}$ with $V_{R}$ the corresponding universal deformation.

(1) For $h \geqslant 0$, there exists a projective $R$-scheme $\mathcal{L}^{\leqslant h}$ such that, for $B$ a finite $\mathbb{Z}_{p}$-algebra, morphisms $\operatorname{Spec} B \rightarrow \mathcal{L}^{\leqslant h}$ correspond to Breuil-Kisin modules $\mathfrak{M}_{B} \subset M_{B}$ of height $\leqslant$ inside the etale $\varphi$-module $M_{B}$ associated with $V_{B}=V_{R} \otimes_{R} B$. 
(2) There exists a closed subscheme $\mathcal{L}_{\text {crys }}^{\leqslant h} \subset \mathcal{L}^{\leqslant h}$ such that:

(a) The scheme-theoretic image of $\mathcal{L}_{\text {crys }}^{\leqslant h} \rightarrow \operatorname{Spec} R$ corresponds to a quotient $R_{\text {crys }}^{\leqslant h}$ of $R$ with the property that, for $E / \mathbb{Q}_{p}$ a finite extension, $x: R \rightarrow E$ factors through $R_{\text {crys }}^{\leqslant h}$ if and only if $V_{E}=V_{R} \otimes_{R} E$ is crystalline with Hodge-Tate weights in $[0, h]$.

(b) The morphism $\mathcal{L}_{\text {crys }}^{\leqslant h} \rightarrow \operatorname{Spec} R_{\text {crys }}^{\leqslant h}$ becomes an isomorphism after inverting $p$.

(c) For $B$ a finite $\mathbb{Z}_{p}$-algebra, the $B$-valued points of $\mathcal{L}_{\text {crys }}^{\leqslant h}$ correspond to those $\mathfrak{M}_{B} \subset M_{B}$ with which, under the identification $\mathfrak{M}_{B} \otimes_{\mathfrak{S}} W\left(C^{b}\right)=$ $V_{B} \otimes_{\mathbb{Z}_{p}} W\left(C^{b}\right)$,

$$
(\sigma-1)(m) \in \mathfrak{M}_{B} \otimes_{\mathfrak{S}}\left[\pi^{\mathrm{b}}\right] \varphi^{-1}(\mu) A_{\mathrm{inf}}
$$

whenever $\sigma \in G_{K}$ and $m \in \mathfrak{M}_{B}$.

(3) Suppose $K / \mathbb{Q}_{p}$ is unramified and $h \leqslant p$. If $\mathfrak{M}_{B}$ corresponds to a B-valued point of $\mathcal{L}_{\text {crys }}^{\leqslant h}$ with $B$ a finite $\mathbb{F}_{p}$-algebra, then $\mathfrak{M}_{B}$ is strongly divisible in the sense of the bullet point above.

(4) Suppose $K / \mathbb{Q}_{p}$ is unramified, $h \leqslant p$, and $V_{\mathbb{F}}$ is cyclotomic-free (this assumption is unnecessary if $h \leqslant p-1)$. Let $\mathcal{L}^{\circ}$ denote the closed subscheme of $\mathcal{L}_{\text {crys }}^{\leqslant h}$ corresponding to the ideal sheaf generated by nilpotent or $p$-torsion elements. Then:

(a) $\mathcal{L}^{\circ}$ is a union of connected components inside $\mathcal{L}_{\text {crys }}^{\leqslant h}$, and the completed local rings of $\mathcal{L}^{\circ}$ at its closed points are formally smooth over $\mathbb{Z}_{p}$.

(b) For each Hodge type $\mathbf{v}$ concentrated in degree $[0, p]$ (that is, for each collection of Hodge-Tate weights contained in $[0, p])$, there exists a union of connected components $\mathcal{L}^{\mathbf{v}} \subset \mathcal{L}^{\circ}$ such that if $R_{\text {crys }}^{\mathbf{v}}$ denotes the quotient of $R$ corresponding to the scheme-theoretic image of $\mathcal{L}^{\mathbf{v}} \rightarrow$ Spec $R$, a homomorphism $x: R \rightarrow E$ factors through $R_{\text {crys }}^{\mathrm{v}}$ if and only if $V_{E}=V_{R} \otimes_{R} E$ is crystalline with Hodge type $\mathbf{v}$.

(c) The morphisms $\mathcal{L}^{\mathbf{v}} \rightarrow$ Spec $R_{\text {crys }}^{\mathbf{v}}$ become isomorphisms after inverting $p$.

Part (2) implies any crystalline deformation $x$ of $V_{\mathbb{F}}$ to $\mathcal{O}$ with Hodge-Tate weights contained in $[0, p]$, where $\mathcal{O}$ is the ring of integers inside a finite extension $E / \mathbb{Q}_{p}$, corresponds to an $\mathcal{O}$-valued point of Spec $R_{\text {crys }}^{\leqslant p}$, which factors uniquely through $\mathcal{L}_{\text {crys }}^{\leqslant p}$. Since $\mathcal{O}$ is $\mathbb{Z}_{p}$-flat and reduced, this $\mathcal{O}$-valued point 
factors through $\mathcal{L}^{\circ}$. If $x^{\prime}$ is another such point that coincides with $x$ on the closed point of $\operatorname{Spec} \mathcal{O}$ (that is a crystalline deformation to $\mathcal{O}$ whose corresponding Breuil-Kisin module is congruent modulo $p$ to that corresponding to $x$ ), then both $x$ and $x^{\prime}$ factor through the same connected component of $\mathcal{L}^{\circ}$. By 4(b), both $x$ and $x^{\prime}$ must have the same Hodge-Tate weights. By $4(\mathrm{a}), \mathcal{L}^{\circ}$ is normal, so in fact $x$ and $x^{\prime}$ lie in the same irreducible component.

We deduce Theorem 1.1.1 by combining this observation with the following lifting result. (Previous results in this vein have been obtained in [11] and [3], with slight variations on the notion of cyclotomic-free.) This is proven by interpreting the lifting strategy of Gee-Liu-Savitt from a geometric point of view, using the formal smoothness obtained in 4(a).

THEOREM 1.2.2. Assume $K / \mathbb{Q}_{p}$ is unramified. Let $V_{\mathbb{F}}$ be a continuous representation of $G_{K}$ on a finite-dimensional $\mathbb{F}$-vector space. Assume that every Jordan-Holder factor of $V_{\mathbb{F}}$ is one-dimensional. Suppose that $\overline{\mathfrak{M}}$ is a strongly divisible Breuil-Kisin module associated with $V_{\mathbb{F}}$. Then $V_{\mathbb{F}}$ admits a crystalline lift, all of whose Jordan-Holder factors are one-dimensional and whose corresponding Breuil-Kisin module equals $\overline{\mathfrak{M}}$ after reducing modulo $p$.

We conclude this introduction by explaining the proof of Theorem 1.2.1. In doing so, we also describe the contents of the various sections in this paper. Parts (1) and (2) are discussed in Section 2. The first is taken from [21, 1.5.1], and the second relies on a key result of Gee-Liu-Savitt and Ozeki, which asserts that the condition in (b) when $B=\mathbb{Z}_{p}$ is a necessary and sufficient condition for a $\mathbb{Z}_{p}$-representation of $G_{K}$ to be crystalline.

Part (3) is addressed in Section 3 and contains the key technical calculations. In Section 4, part (4) is proved. The idea is to use part (3) to produce a homomorphism from the mod $p$ tangent space of $\mathcal{L}_{\text {crys }}^{\leqslant p}$ at closed points into extension groups of strongly divisible Breuil-Kisin modules. The dimensions of these extension groups have been computed in [3] (generalizing the calculations mentioned above from [16]). The cyclotomic-freeness assumption allows us to control the kernel of these maps. We deduce these tangent spaces have dimension $\leqslant \operatorname{dim} \mathcal{L}_{\text {crys }}^{\leqslant h}[1 / p]=\operatorname{dim} \operatorname{Spec} R_{\text {crys }}^{\leqslant p}[1 / p]$ (this can be compared with the bullet above since these dimensions coincide with dimensions of certain spaces of crystalline extensions).

Finally, in Section 5, we put all these results together and prove Theorems 1.1.1 and 1.2.2. We also discuss some possible improvements to this strategy (focusing on removing the dimension assumptions on the Jordan-Holder factors in Theorem 1.2.2). In the last part, we explain how, in certain nice situations, the geometries of $\mathcal{L}^{\mathbf{v}}$ and $R_{\text {crys }}^{\mathbf{v}}$ are particularly closely related. We illustrate this 
for two-dimensional representations of $K=\mathbb{Q}_{p}$, recovering some results from [23] and [27] on two-dimensional crystalline deformation rings of $G_{\mathbb{Q}_{p}}$ with Hodge-Tate weights contained in $[0, p]$.

\section{Crystalline deformation rings}

\subsection{Integral $p$-adic Hodge theory}

2.1.1. Let $k$ be a finite field of characteristic $p$ and let $K_{0}=W(k)[1 / p]$. Fix $K$ a totally ramified extension of $K_{0}$ of degree $e$. Also fix a uniformizer $\pi$ of $K$ and a compatible system $\pi^{1 / p^{\infty}}$ of $p$ th power roots of $\pi$ in an algebraic closure $\bar{K}$ of $K$. Let $E(u) \in W(k)[u]$ denote the minimal polynomial of $\pi$ over $K_{0}$.

The ring $\mathfrak{S}=W(k)[[u]]$ is equipped with a Frobenius $\varphi$, which acts on $W(k)$ as the usual Witt vector Frobenius and which sends $u \mapsto u^{p}$. This Frobenius extends uniquely to a Frobenius on $\mathcal{O}_{\mathcal{E}}$, the $p$-adic completion of $\mathfrak{S}[1 / u]$, which we again denote by $\varphi$.

Let $C$ be the completion of $\bar{K}$ with integers $\mathcal{O}_{C}$. The inverse limit of the system

$$
\mathcal{O}_{C} / p \leftarrow \mathcal{O}_{C} / p \leftarrow \mathcal{O}_{C} / p \leftarrow \cdots
$$

(with transition maps given by $x \mapsto x^{p}$ ) is denoted by $\mathcal{O}_{C^{b}}$. By construction, the $p$ th power map on $\mathcal{O}_{C^{b}}$ is an automorphism. The obvious map $\lim _{x \mapsto x^{p}} \mathcal{O}_{C} \rightarrow \mathcal{O}_{C^{b}}$ is a multiplicative bijection, which allows us to equip $\mathcal{O}_{C^{b}}$ with a valuation $v^{b}$ as follows. If $v$ denotes the valuation on $\mathcal{O}_{C}$ normalized so that $v(p)=1$, then $v^{b}(x):=v\left(x^{\sharp}\right)$, where $x^{\sharp} \in \mathcal{O}_{C}$ is the image of $x$ under the projection $\mathcal{O}_{C^{b}}=$ $\underset{\lim }{\longleftarrow} \mathcal{O}_{C} \rightarrow \mathcal{O}_{C}$ onto the first coordinate. This makes $\mathcal{O}_{C^{b}}$ a complete valuation ring with a field of fractions $C^{\text {b }}$. The continuous $G_{K}$-action on $\mathcal{O}_{C}$ induces continuous $G_{K}$-actions on $\mathcal{O}_{C^{b}}$ and $C^{\text {b }}$.

Let $A_{\text {inf }}=W\left(\mathcal{O}_{C^{b}}\right)$. By functoriality of the Witt vector construction, the $G_{K}$-action on $\mathcal{O}_{C^{\dot{b}}}$ transfers to a $G_{K}$-action on $A_{\text {inf }}$. Likewise, we obtain a $G_{K}$-action on $W\left(C^{b}\right)$. We also obtain Frobenius endomorphisms on $A_{\text {inf }}$ and $W\left(C^{b}\right)$ lifting the $p$ th power maps on $\mathcal{O}_{C^{b}}$ and $C^{b}$. These endomorphisms commute with the $G_{K}$-actions.

The compatible system of $p$ th power roots of $\pi \in K$ gives rise to an element $\pi^{b} \in \mathcal{O}_{C^{b}}$ with $v^{b}\left(\pi^{b}\right)=1 / e$. The map of $W(k)$-algebras $\mathfrak{S} \rightarrow A_{\text {inf }}$ sending $u \mapsto\left[\pi^{b}\right]$ (where $[\cdot]$ denotes the Teichmuller map) is an embedding compatible with the Frobenius on either ring. This map extends to a Frobenius compatible embedding $\mathcal{O}_{\mathcal{E}} \rightarrow W\left(C^{b}\right)$, where $\mathcal{O}_{\mathcal{E}}$ denotes the $p$-adic completion of $\mathfrak{S}[1 / u]$.

2.1.2. Let $V$ be a finitely generated $\mathbb{Z}_{p}$-module equipped with a continuous $\mathbb{Z}_{p}$-linear action of $G_{K_{\infty}}$. The results of [9] assert that there exists a unique finitely 
generated $\mathcal{O}_{\mathcal{E}}$-submodule $M \subset V \otimes_{\mathbb{Z}_{p}} W\left(C^{b}\right)$ such that

$$
M \otimes_{\mathcal{O}_{\mathcal{E}}} W\left(C^{b}\right)=V \otimes_{\mathbb{Z}_{p}} W\left(C^{b}\right)
$$

and such that the $W\left(C^{b}\right)$-semilinear extension of the $G_{K_{\infty}}$-action on $V$ fixes $M$ and such that the restriction of the trivial $W\left(C^{b}\right)$-semilinear Frobenius on $V$ induces an isomorphism $M \otimes_{\mathcal{O}_{\mathcal{E}}, \varphi} \mathcal{O}_{\mathcal{E}}=: \varphi^{*} M \stackrel{\sim}{\rightarrow} M$. The construction of $M$ is functorial in $V$. In particular, if $V$ admits a $G_{K_{\infty}}$-equivariant $\mathbb{Z}_{p}$-linear action of a $\mathbb{Z}_{p}$-algebra $A$, then $M$ can be viewed as a module over $\mathcal{O}_{\mathcal{E}, A}=\mathcal{O}_{\mathcal{E}} \otimes_{\mathbb{Z}_{p}} A$.

DEFINITION 2.1.4. If $V$ is a finite free $\mathbb{Z}_{p}$-module equipped with a continuous $\mathbb{Z}_{p}$-linear action of $G_{K}$, and if $M$ is associated with $\left.V\right|_{G_{K_{\infty}}}$ as in 2.1.2, then $V$ has $E$-height $\leqslant h$ if there exists a $\varphi$-stable finite free $\mathfrak{S}$-submodule $\mathfrak{M} \subset M$ such that (i) the induced map $\varphi^{*} \mathfrak{M}=\mathfrak{M} \otimes_{\mathfrak{S}, \varphi} \mathfrak{S} \rightarrow \mathfrak{M}$ has cokernel killed by $E(u)^{h}$ and (ii) there is an equality $\mathfrak{M} \otimes_{\mathfrak{S}} \mathcal{O}_{\mathcal{E}}=M$.

The association of $\mathfrak{M}$ with a representation of $E$-height $\leqslant h$ is a fully faithful functor; see [20, 2.1.12]. In particular, there exists at most one $\mathfrak{M} \subset M$ as above; we call this the Breuil-Kisin module associated with $V$.

2.1.5. As usual, let $\mathbb{Z}_{p}(1)$ denote the free rank-one $\mathbb{Z}_{p}$-module consisting of compatible systems of $p$ th power roots of unity in $\bar{K}$. Consider the ring of $p$-adic periods $B_{\mathrm{dR}}^{+}$defined in [10]. There is a homomorphism $\mathbb{Z}_{p}(1) \rightarrow B_{\mathrm{dR}}^{+}$sending $\xi \mapsto \log ([\xi]):=\sum_{n \geqslant 1}(-1)^{n+1}\left(([\xi]-1)^{n} / n\right)$. Fix a $\mathbb{Z}_{p}$-generator $\epsilon$ of $\mathbb{Z}_{p}(1)$ and set $t=\log ([\epsilon])$. We also write $\mu=[\epsilon]-1 \in A_{\text {inf }}$.

As in [8, III.1], let $A_{\max } \subset B_{\mathrm{dR}}^{+}$be the subring of elements, which can be written as $\sum_{n \geqslant 0} x_{n}\left(\left[\pi^{\mathrm{b}}\right]^{e n} / p^{n}\right) \in B_{\mathrm{dR}}^{+}$with $\left(x_{n}\right)_{n \geqslant 0}$ a sequence in $A_{\text {inf }}$ converging $p$-adically to zero. Then $B_{\max }^{+}=A_{\max }[1 / p]$ and $B_{\max }=B_{\max }^{+}[1 / t]$. The Frobenius on $A_{\text {inf }}$ extends to each of these rings.

If $V$ is a finite free $\mathbb{Z}_{p}$-module equipped with a continuous $\mathbb{Z}_{p}$-linear action of $G_{K}$, then $V \otimes_{\mathbb{Z}_{p}} \mathbb{Q}_{p}$ is crystalline if and only if the $K_{0}$-vector space $D_{\text {crys }}(V):=$ $\left(V \otimes_{\mathbb{Z}_{p}} B_{\max }\right)^{G_{K}}$ has dimension equal to $\operatorname{rank}_{\mathbb{Z}_{p}} V$. (The usual definition of a crystalline representation is made using the period ring $B_{\text {crys }}$. If $A_{\text {crys }} \subset B_{\mathrm{dR}}$ consists of elements of the form $\sum_{n \geqslant 0} x_{n}\left(\left[\pi^{b}\right]^{e n} / n !\right)$ with $x_{n} \in A_{\text {inf }}$ converging $p$-adically to zero, then

$$
B_{\text {crys }}^{+}=A_{\text {crys }}\left[\frac{1}{p}\right] \quad \text { and } \quad B_{\text {crys }}=B_{\text {crys }}^{+}\left[\frac{1}{t}\right] .
$$

Since $v_{p}(n !) \leqslant n$ and $n \leqslant v_{p}((p n) !)$, we see that $\varphi\left(A_{\max }\right) \subset A_{\text {crys }} \subset A_{\text {max }}$. Thus $\varphi\left(B_{\text {max }}\right) \subset B_{\text {crys }} \subset B_{\max }$. Using this one, we see that $\left(V \otimes_{\mathbb{Z}_{p}} B_{\max }\right)^{G_{K}}=$ $\left(V \otimes_{\mathbb{Z}_{p}} B_{\text {crys }}\right)^{G_{K}}$.) 
2.1.6. Suppose $V$ is a representation of $E$-height $\leqslant h$ with corresponding BreuilKisin module $\mathfrak{M}$. Set $D=(\mathfrak{M} / u \mathfrak{M}) \otimes_{W(k)} K_{0}$. This is a $K_{0}$-vector space equipped with a bijective Frobenius $\varphi^{*} D \stackrel{\sim}{\rightarrow} D$. We claim there exist $\varphi, G_{K_{\infty}}$-equivariant identifications

$$
D \otimes_{K_{0}} B_{\max } \cong \mathfrak{M} \otimes_{\mathfrak{S}} B_{\max } \cong V \otimes_{\mathbb{Z}_{p}} B_{\max },
$$

where $G_{K_{\infty}}$ is made to act trivially on $D$. The right-hand identification follows from the next lemma since $A_{\mathrm{inf}}[1 / \mu]$ is a subring of $B_{\max }$.

LEMMA 2.1.8. Let $V$ be a representation of E-height $\leqslant h$ and $\mathfrak{M}$ the corresponding Breuil-Kisin module. Write $\mathfrak{M}^{\varphi}$ for the image of $\varphi^{*} \mathfrak{M} \rightarrow \mathfrak{M}$. Then there exists a $\varphi, G_{K_{\infty}}$-equivariant identification

$$
\mathfrak{M}^{\varphi} \otimes_{\mathfrak{S}} A_{\text {inf }}\left[\frac{1}{\mu}\right] \cong V \otimes_{\mathfrak{S}} A_{\text {inf }}\left[\frac{1}{\mu}\right],
$$

which recovers (2.1.3) after tensoring with $W\left(C^{b}\right)$.

Proof. This follows by applying [5, Lemma 4.26] to the Breuil-Kisin-Fargues module $\mathfrak{M}^{\varphi} \otimes_{\mathfrak{S}} A_{\text {inf }}=\varphi(\mathfrak{M}) \otimes_{\varphi(\mathfrak{S})} A_{\text {inf }}$. (Note that in loc. cit., $\mathfrak{S}$ is viewed as a subring of $A_{\text {inf }}$ via $u \mapsto\left[\pi^{b}\right]^{p}$, which is different from our embedding. This is the reason why $\mathfrak{M}^{\varphi}$ appears rather than $\mathfrak{M}$.)

For the left-hand side of (2.1.7), let $\mathcal{O}^{\text {rig }} \subset K_{0}[[u]]$ denote the subring of power series converging on the open unit disk, and consider $\lambda=\prod_{n=0}^{\infty} \varphi^{n}(E(u) / E(0)) \in$ $\mathcal{O}^{\text {rig }}$. In $[20,1.2 .6]$, a $\varphi$-equivariant inclusion

$$
D \otimes_{K_{0}} \mathcal{O}^{\text {rig }} \hookrightarrow \mathfrak{M}^{\varphi} \otimes_{\mathfrak{S}} \mathcal{O}^{\text {rig }}
$$

is constructed, which is an isomorphism modulo $u$ and which becomes an isomorphism after inverting $\varphi(\lambda)$. It is also $G_{K_{\infty}}$-equivariant, for the trivial $G_{K_{\infty}}$-action on both sides. Since the inclusion $\mathfrak{S} \rightarrow A_{\text {inf }}$ extends to an embedding $\mathcal{O}^{\text {rig }} \rightarrow B_{\max }^{+}$, which maps $\varphi(\lambda)$ onto a unit in $B_{\max }^{+}$, we obtain the left-hand side of (2.1.7).

We can now formulate the main result of [20]. See also [24, 1.2.1].

Proposition 2.1.10 (Kisin). If $V$ is a $G_{K}$-stable $\mathbb{Z}_{p}$-lattice inside a crystalline representation with Hodge-Tate weights in $[0, h]$, then $V$ is of E-height $\leqslant h$. (Our Hodge-Tate weights are normalized so that the cyclotomic character has weight -1.) Furthermore: 
(1) $D_{\text {crys }}(V) \subset V \otimes_{\mathbb{Z}_{p}} B_{\max }$ is identified with $D$ under (2.1.7).

(2) Tensoring (2.1.9) with the map $\mathcal{O}^{\text {rig }} \rightarrow K$ given by $u \mapsto \pi$ identifies $\mathfrak{M}^{\varphi} / E(u) \mathfrak{M}^{\varphi}$ with an $\mathcal{O}_{K}$-lattice inside $D_{\text {crys }}(V)_{K}=D_{\text {crys }}(V) \otimes_{K_{0}} K$. Via the inclusion $B_{\max } \otimes_{K_{0}} K \rightarrow B_{\mathrm{dR}}$ we identify $D_{\text {crys }}(V)_{K}=\left(V \otimes_{\mathbb{Q}_{p}} B_{\mathrm{dR}}\right)^{G_{K}}$ and, under this identification, the surjection $\mathfrak{M}^{\varphi} \rightarrow D_{\text {crys }}(V)_{K}$ induces a map of $\mathfrak{M}^{\varphi} \cap E(u)^{i} \mathfrak{M}$ into

$$
F^{i} D_{\text {crys }}(V)_{K}:=\left(V \otimes_{\mathbb{Q}_{p}} t^{i} B_{\mathrm{dR}}^{+}\right)^{G_{K}},
$$

which becomes surjective after inverting $p$.

Not every finite $E$-height representation is crystalline; indeed in [12, 1.1.13], it is shown that $V$ has finite $E$-height if and only if $\left.V\right|_{G_{K_{m}}}$ is semistable, where $K_{m}=K\left(\pi^{1 / p^{m}}\right)$ for a suitably large $m$. The starting point of this article is a description identifying which finite $E$-height representations are crystalline. To explain this, fix a representation $V$ of finite $E$-height with associated BreuilKisin module $\mathfrak{M}$. Using Lemma 2.1 .8 or (2.1.3), we obtain a $\varphi, G_{K_{\infty}}$-equivariant identification

$$
\mathfrak{M} \otimes_{\mathfrak{S}} W\left(C^{b}\right) \cong V \otimes_{\mathbb{Z}_{p}} W\left(C^{b}\right) .
$$

The $G_{K}$-actions on $V$ and $W\left(C^{b}\right)$ therefore transfer to a $\varphi$-equivariant $G_{K}$-action on $\mathfrak{M} \otimes_{\mathfrak{S}} W\left(C^{b}\right)$.

THEOREM 2.1.12 (Gee-Liu-Savitt, Ozeki). Let $V$ be a finite free $\mathbb{Z}_{p}$-module with a continuous $\mathbb{Z}_{p}$-linear action of $G_{K}$. Then the following are equivalent:

(1) $V \otimes_{\mathbb{Z}_{p}} \mathbb{Q}_{p}$ is crystalline with Hodge-Tate weights in $[0, h]$.

(2) $V$ is of E-height $\leqslant h$ and the $G_{K}$-action on $\mathfrak{M} \otimes_{\mathfrak{S}} W\left(C^{b}\right)$ induced from (2.1.11) is such that $(\sigma-1)(m) \in \mathfrak{M} \otimes_{\mathfrak{S}}\left[\pi^{\mathrm{b}}\right] \varphi^{-1}(\mu) A_{\text {inf }}$ for every $m \in \mathfrak{M}$ and $\sigma \in G_{K}$.

That (1) implies (2) is essentially [16, 4.10], while the converse is proven in [26, Theorem 21]. As both these results are not formulated as we need (and also because they assume that $p>2$ ), we devote the rest of this section to a proof of the theorem. Our argument that (1) implies (2) is essentially the same as that in [16], but our proof of the converse differs from Ozeki's.

Proof that (2) implies (1) in Theorem 2.1.12. In fact, we prove something stronger. Namely, consider $V$ and $\mathfrak{M}$ as in 2.1.6 and suppose the $G_{K}$-action on $V$ is such that, when transferred to $\mathfrak{M} \otimes_{\mathfrak{S}} B_{\max }$ via (2.1.7),

$$
(\sigma-1)(m) \in \mathfrak{M} \otimes_{\mathfrak{S}}\left[\pi^{\mathrm{b}}\right] A_{\text {inf }}
$$


for every $m \in \mathfrak{M}$ and $\sigma \in G_{K}$. Then we show $V \otimes_{\mathbb{Z}_{p}} \mathbb{Q}_{p}$ is crystalline. For this, it suffices to show the $G_{K}$-action is trivial on $D$. To this end, let $S_{\max } \subset \mathcal{O}^{\text {rig }}$ denote the subring $W(k)\left[\left[u, u^{e} / p\right]\right] \cap \mathcal{O}^{\text {rig }}$. Clearly, the inclusion $\mathcal{O}^{\text {rig }} \rightarrow B_{\max }^{+}$maps $S_{\max }$ into $A_{\max }$. Recall that a power series $\sum a_{i} u^{i}$ with $a_{i} \in K_{0}$ lies in $\mathcal{O}^{\text {rig }}$ if and only if $v_{p}\left(a_{i}\right)+i r \rightarrow 0$ for any $r>0$. This series is contained in $S_{\max }$ if furthermore $v\left(a_{i}\right)+i / e \geqslant 0$. By taking $r=1 / e$, we see $S_{\max }[1 / p]=\mathcal{O}^{\text {rig }}$, and so we can choose a $W(k)$-lattice $D^{\circ} \subset D$ so that every $d \in D^{\circ}$ can be written as $\sum s_{i} m_{i}$ with $s_{i} \in S_{\max }$ and $m_{i} \in \mathfrak{M}$. If $s \in S_{\max }$ and $\sigma \in G_{K}$, then $(\sigma-1)(s) \in\left(\left[\pi^{\triangleright}\right] / p\right) A_{\max }$ since $G_{K}$ acts trivially on the constant term. From this and (2.1.13), we deduce that

$$
(\sigma-1)(d)=\sum\left(\sigma\left(s_{i}\right)-s_{i}\right) \sigma\left(m_{i}\right)+\sum s_{i}\left(\sigma\left(m_{i}\right)-m_{i}\right) \in \mathfrak{M} \otimes_{\mathfrak{S}} \frac{\left[\pi^{\mathrm{b}}\right]}{p} A_{\max }
$$

for any $d=\sum s_{i} m_{i} \in D^{\circ}$. There exists an $m \in \mathbb{Z}$ such that $\varphi^{-1}\left(D^{\circ}\right) \subset\left(1 / p^{m}\right) D^{\circ}$. Thus $\varphi^{-n}\left(D^{\circ}\right) \subset\left(1 / p^{n m}\right) D^{\circ}$ for $n \geqslant 1$. Since the $G_{K}$-action is $\varphi$-equivariant, we have

$$
\begin{aligned}
(\sigma-1)(d) & =\varphi^{n}\left((\sigma-1)\left(\varphi^{-n}(d)\right)\right) \in \varphi^{n}\left(\mathfrak{M} \otimes_{\mathfrak{S}} \frac{\left[\pi^{\mathrm{b}}\right]}{p^{n m+1}} A_{\max }\right) \\
& \subset \mathfrak{M} \otimes_{\mathfrak{S}} \frac{\left[\pi^{\mathrm{b}}\right]^{p^{n}}}{p^{n m+1}} A_{\max }
\end{aligned}
$$

whenever $d \in D^{\circ}$. However, $\left[\pi^{\mathrm{b}}\right]^{p^{n}} / p^{n m+1} \in p^{p^{n}-n m-1} A_{\max }$ and so, since $A_{\max }$ is $p$-adically complete, it must be that $(\sigma-1)(d)=0$.

Now we show (1) implies (2). One of the advantages of using $B_{\max }^{+}$is that its topology is better behaved than that of $B_{\text {crys }}^{+}$. In particular, we have the following lemma.

LEMMA 2.1.14. Equip $B_{\max }^{+}$with the topology making $\left(p^{n} A_{\max }\right)_{n \geqslant 0}$ a basis of open neighbourhoods of 0 . Then $B_{\max }^{+}$is complete and any principal ideal $a B_{\max }^{+} \subset B_{\max }^{+}$is closed.

Proof. Completeness is immediate since $A_{\max }$ is $p$-adically complete. To check $a B_{\max }^{+}$is closed, consider a sequence $b_{i} \in a B_{\max }^{+}$converging to $b \in B_{\max }^{+}$. We must show $b \in a B_{\max }^{+}$. Since $B_{\max }^{+}$is a domain, it suffices to show $b_{i} / a$ converges in $B_{\max }^{+}$. This follows from [8, III.2.1], which asserts that if $\|x\|=\inf _{n \mid p^{n} x \in A_{\max }} p^{n}$, then $p^{-1}\|x\|\|y\| \leqslant\|x y\|$. Hence $\left\|\left(b_{i} / a\right)-\left(b_{j} / a\right)\right\| \leqslant(p /\|a\|)\left\|b_{i}-b_{j}\right\|$, and so as $B_{\max }^{+}$is complete, $b_{i} / a$ converges.

For $\sigma \in G_{K}$, consider $\epsilon(\sigma) \in \mathbb{Z}_{p}(1)$ defined by $\epsilon(\sigma)_{n}=\sigma\left(\pi^{1 / p^{n}}\right) / \pi^{1 / p^{n}}$. 
Lemma 2.1.15. Suppose $V \otimes_{\mathbb{Z}_{p}} \mathbb{Q}_{p}$ is crystalline and that $\mathfrak{M}$ is the Breuil-Kisin module associated with $V$. Define a differential operator $\mathcal{N}$ over $\partial=u(d / d u)$ on $\mathfrak{M} \otimes_{\mathfrak{S}} \mathcal{O}^{\text {rig }}[1 / \lambda]=D \otimes_{K_{0}} \mathcal{O}^{\text {rig }}[1 / \lambda]$ by asserting $\mathcal{N}(d)=0$ for all $d \in D$. Then

$$
\sigma(m)=\sum_{n \geqslant 0} \mathcal{N}^{n}(m) \otimes \frac{(-\log ([\epsilon(\sigma)]))^{n}}{n !}
$$

for $m \in \mathfrak{M}^{\varphi} \otimes_{\mathfrak{S}} \mathcal{O}^{\text {rig }}[1 / \varphi(\lambda)]$ and $\sigma \in G_{K}$.

Proof. Since $\mathfrak{M}^{\varphi} \otimes_{\mathfrak{S}} \mathcal{O}^{\text {rig }}[1 / \varphi(\lambda)]=D \otimes_{K_{0}} \mathcal{O}^{\text {rig }}[1 / \varphi(\lambda)]$, it is enough to consider $m=f d$ with $d \in D$ and $f \in \mathcal{O}^{\text {rig }}[1 / \varphi(\lambda)]$. By definition, $\mathcal{N}^{n}(f d)=\partial^{n}(f) d$. By (1) of Proposition 2.1.10, we identify $D=D_{\text {crys }}(V)$ and the $G_{K}$-action on $V$ fixes $D$; hence $\sigma(f d)=\sigma(f) d$. The lemma therefore reduces to checking that

$$
\sum_{n \geqslant 0} \frac{(-\log ([\epsilon(\sigma)]))^{n}}{n !} \partial^{n}(f)
$$

converges in $B_{\max }^{+}$to $\sigma(f)$. It suffices to consider $f=u^{i}$. Then $\sigma(f)=$ $\left[\epsilon(\sigma)^{i}\right] u^{i}$. On the other hand, using that $\partial^{n}\left(u^{i}\right)=(-i)^{n} u^{i}$, we see (2.1.16) equals $\exp \left(\log \left([\epsilon(\sigma)]^{i}\right)\right) u^{i}$. If this sum converges, it will do so to $[\epsilon(\sigma)]^{i} u^{i}$, which proves the lemma.

To show convergence, it is enough to show $\left(\log ([\epsilon(\sigma)])^{n}\right) / n$ ! lies in $A_{\max }$ and in this ring converges $p$-adically to zero. Note that $\log ([\epsilon(\sigma)])=\alpha t$ for some $\alpha \in \mathbb{Z}_{p}$. The proof of [8, III.3.9] shows that $t \in p A_{\max }$ if $p>2$ and $t \in p^{2} A_{\max }$ if $p=2$. Convergence of $\left(\log ([\epsilon(\sigma)])^{n}\right) / n$ ! then follows because $p^{n} / n ! \in \mathbb{Z}_{p}$ converges $p$-adically to zero when $p>2$, and $p^{2 n} / n$ ! converges $p$-adically to zero when $p=2$.

Proof that (1) implies (2) in Theorem 2.1.12. It suffices to prove, for $m \in \varphi(\mathfrak{M})$ and $\sigma \in G_{K}$, that $(\sigma-1)(m) \in \mathfrak{M}^{\varphi} \otimes_{\mathfrak{S}}\left[\pi^{\mathrm{b}}\right]^{p} \mu A_{\text {inf }}$. Since $A_{\text {inf }}[1 / \mu]$ is $G_{K}$-stable, Lemma 2.1.8 ensures that $(\sigma-1)(m) \in \mathfrak{M}^{\varphi} \otimes_{\mathfrak{S}} A_{\text {inf }}[1 / \mu]$.

On the other hand, we know from the previous lemma that

$$
(\sigma-1)(m)=\sum_{n \geqslant 1} \mathcal{N}^{n}(m) \otimes \frac{(-\log ([\epsilon(\sigma)]))^{n}}{n !} .
$$

Since $\partial \circ \varphi=p \varphi \circ \partial$, the operator $\mathcal{N}$ satisfies $\mathcal{N} \varphi=p \varphi \mathcal{N}$, and so

$$
\mathcal{N}^{n}(m) \in \varphi\left(\mathcal{N}\left(\mathfrak{M} \otimes_{\mathfrak{S}} \mathcal{O}^{\text {rig }}\left[\frac{1}{\lambda}\right]\right)\right)
$$

for $n \geqslant 1$. By the definition of $\mathcal{N}$, we have $\mathcal{N}\left(\mathfrak{M} \otimes_{\mathfrak{S}} \mathcal{O}^{\text {rig }}[1 / \varphi(\lambda)]\right) \subset \mathfrak{M} \otimes_{\mathfrak{S}}$ $u \mathcal{O}^{\text {rig }}[1 / \lambda]$. Therefore $\mathcal{N}^{n}(m) \in \mathfrak{M}^{\varphi} \otimes_{\mathfrak{S}}\left[\pi^{b}\right]^{p} B_{\max }^{+}$. Since $\log ([\epsilon(\sigma)]) \in t A_{\max }=$ $\mu A_{\max }$ (the equality follows from [8, III.3.9]), each term of (2.1.17) is contained 
in $\mathfrak{M}^{\varphi} \otimes_{\mathfrak{S}}\left[\pi^{b}\right]^{p} \mu B_{\max }^{+}$. Lemma 2.1.14 implies the entire sum (2.1.17) is contained in $\mathfrak{M}^{\varphi} \otimes_{\mathfrak{S}}\left[\pi^{\mathrm{b}}\right]^{p} \mu B_{\max }^{+}$.

To complete the proof, it suffices to show that

$$
A_{\text {inf }}\left[\frac{1}{\mu}\right] \cap\left[\pi^{\triangleright}\right]^{p} \mu B_{\max }^{+}=\left[\pi^{b}\right]^{p} \mu A_{\text {inf }} .
$$

This follows from the next two facts. The first is that if $a \in A_{\text {inf }} \cap\left[\pi^{b}\right]^{p} B_{\max }^{+}$, then $a \in\left[\pi^{b}\right]^{p} A_{\text {inf. }}$. This is proven with $B_{\max }^{+}$replaced by $B_{\text {crys }}^{+}$in [25, Lemma 3.2.2]. Using that $\varphi\left(B_{\max }^{+}\right) \subset B_{\text {crys }}^{+}$, we deduce the same applies for $B_{\max }^{+}$. The second

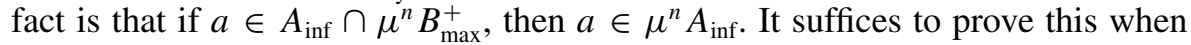
$n=1$. The homomorphism $\theta: A_{\text {inf }} \rightarrow \mathcal{O}_{C}$ given by $\sum\left[x_{i}\right] p^{i} \mapsto \sum x_{i}^{\sharp} p^{i}$ extends to $\theta: B_{\max }^{+} \rightarrow C$ and, since $\theta\left(\varphi^{n}(\mu)\right)=0$, we must have $\theta\left(\varphi^{n}(a)\right)=0$ for all $n \geqslant 0$. The claim then follows from [10, Proposition 5.1.3], which states that $\left\{a \in A_{\text {inf }} \mid \varphi^{n}(a) \in \operatorname{ker} \theta\right.$ for all $\left.n \geqslant 0\right\}=\mu A_{\text {inf }}$.

\subsection{The locus of crystalline Breuil-Kisin modules}

2.2.1. Let $A$ be an Artin local ring with finite residue field $\mathbb{F}$ of characteristic $p$. Suppose $V_{A}$ is a finite free $A$-module equipped with a continuous $A$-linear $G_{K_{\infty}}$-action.

Since $A$ is a finite $\mathbb{Z}_{p}$-module, as in 2.1.2 we obtain an $\mathcal{O}_{\mathcal{E}, A}=\mathcal{O}_{\mathcal{E}} \otimes_{\mathbb{Z}_{p}}$ $A$-module $M_{A}$ equipped with an isomorphism $\varphi^{*} M_{A} \stackrel{\sim}{\rightarrow} M_{A}$ such that there exists a $\varphi, G_{K_{\infty}}$-equivariant identification (2.1.3). Since $V_{A}$ is $A$-free, $M_{A}$ is $\mathcal{O}_{\mathcal{E}, A}$-free; see $[22,1.2 .7]$. For any $A$-algebra $B$, set $M_{B}=M_{A} \otimes_{A} B$ and $V_{B}=V_{A} \otimes_{A} B$.

Definition 2.2.2. For any $A$-algebra $B$, define $\mathcal{L}^{\leqslant h}\left(V_{B}\right)$ to be the set of finite projective $\mathfrak{S}_{B}=\mathfrak{S} \otimes_{A} B$-submodules $\mathfrak{M}_{B} \subset M_{B}$ satisfying $\mathfrak{M}_{B} \otimes_{\mathfrak{S}} \mathcal{O}_{\mathcal{E}}=M_{B}$ and, if $\mathfrak{M}_{B}^{\varphi}$ denotes the image of $\varphi^{*} \mathfrak{M}_{B}$ under $\varphi^{*} M_{B} \rightarrow M_{B}$, satisfying

$$
E(u)^{h} \mathfrak{M}_{B} \subset \mathfrak{M}_{B}^{\varphi} \subset \mathfrak{M}_{B}
$$

If $B \rightarrow B^{\prime}$ is a map of $A$-algebras and $\mathfrak{M}_{B} \in \mathcal{L}^{\leqslant h}\left(V_{B}\right)$, then $\mathfrak{M}_{B} \otimes_{B} B^{\prime}$ is a finite projective $\mathfrak{S}_{B^{\prime}}$-submodule of $M_{B^{\prime}}$ and $\varphi^{*}\left(\mathfrak{M}_{B} \otimes_{B} B^{\prime}\right) \rightarrow\left(\mathfrak{M}_{B} \otimes_{B} B^{\prime}\right)$ has cokernel killed by $E^{h}$. (Note $\mathfrak{M}_{B} \otimes_{\mathfrak{S}_{B}} \mathfrak{S}_{B^{\prime}}=\mathfrak{M}_{B} \otimes_{\mathfrak{S}_{B}}\left(\mathfrak{S}_{B} \otimes_{B^{\prime}} B\right)=\mathfrak{M}_{B} \otimes_{B^{\prime}} B$.) Since $\mathcal{O}_{\mathcal{E}} \otimes_{\mathfrak{S}}\left(\mathfrak{M}_{B} \otimes_{B} B^{\prime}\right) \cong M_{B} \otimes_{B} B^{\prime}$, we have that $\mathfrak{M}_{B} \otimes_{B} B^{\prime} \in \mathcal{L}^{\leqslant h}\left(V_{B^{\prime}}\right)$. Thus $B \mapsto \mathcal{L}^{\leqslant h}\left(V_{B}\right)$ is a functor on $A$-algebras.

The functor $\mathcal{L}^{\leqslant h}$ was introduced by Kisin. In [21, 1.3], he proves the following proposition. 
Proposition 2.2.3 (Kisin). The functor $B \mapsto \mathcal{L}^{\leqslant h}\left(V_{B}\right)$ is represented by a projective $A$-scheme $\mathcal{L}_{A}^{\leqslant h}$. If $A \rightarrow A^{\prime}$ is a map of Artin local rings with finite residue field, then there are functorial isomorphisms $\mathcal{L}_{A}^{\leqslant h} \otimes_{A} A^{\prime} \cong \mathcal{L}_{A^{\prime}}^{\leqslant h}$. Furthermore, $\mathcal{L}_{A}^{\leqslant h}$ is equipped with a very ample line bundle, which is similarly functorial in A.

2.2.4. Now suppose $V_{A}$ is a finite free $A$-module equipped with a continuous $A$-linear action of $G_{K}$. Apply the previous discussion to $\left.V_{A}\right|_{G_{K_{\infty}}}$. If $B$ is an $A$-algebra and $\mathfrak{M}_{B} \in \mathcal{L}^{\leqslant h}\left(V_{B}\right)$, then (2.1.3) induces a $\varphi, G_{K_{\infty}}$-equivariant isomorphism

$$
\mathfrak{M}_{B} \otimes_{\mathfrak{S}} W\left(C^{b}\right) \cong M_{B} \otimes_{\mathcal{O}_{\mathcal{E}}} W\left(C^{b}\right) \cong V_{B} \otimes_{\mathbb{Z}_{p}} W\left(C^{b}\right) .
$$

The $G_{K}$-action on $V_{B}$ and $W\left(C^{b}\right)$ provides an action of $G_{K}$ on $\mathfrak{M}_{B} \otimes_{\mathfrak{S}} W\left(C^{b}\right)$.

Definition 2.2.6. For any $A$-algebra $B$, let $\mathcal{L}_{\text {crys }}^{\leqslant h}\left(V_{B}\right)$ denote the set of $\mathfrak{M}_{B} \in$ $\mathcal{L}^{\leqslant h}\left(V_{B}\right)$ such that the $G_{K}$-action on $\mathfrak{M}_{B} \otimes_{\mathfrak{S}} W\left(C^{b}\right)$ given by (2.2.5) satisfies

$$
(\sigma-1)(m) \in \mathfrak{M}_{B} \otimes_{\mathfrak{S}}\left[\pi^{b}\right] \varphi^{-1}(\mu) A_{\text {inf }}
$$

for all $m \in \mathfrak{M}_{B}$ and all $\sigma \in G_{K}$. Again $B \mapsto \mathcal{L}_{\text {crys }}^{\leqslant h}\left(V_{B}\right)$ is a functor on $A$-algebras.

We shall prove that $B \mapsto \mathcal{L}_{\text {crys }}^{\leqslant h}\left(V_{B}\right)$ is represented by a closed subscheme of $\mathcal{L}^{\leqslant h}$. First, we need some lemmas.

LEMMA 2.2.7. Let $Q$ be a flat $\mathbb{Z}_{p}$-module and $A$ a $\mathbb{Z}_{p}$-algebra with $p^{n} A=0$ for some $n \geqslant 0$. For any $x \in A \otimes_{\mathbb{Z}_{p}} Q$, there exists a smallest ideal $I(x) \subset A$ such that $x \in I(x) \otimes_{\mathbb{Z}_{p}} Q$.

Proof. We shall show there exists a smallest $\mathbb{Z}_{p}$-submodule $M(x) \subset A$ such that $M(x) \otimes_{\mathbb{Z}_{p}} Q$ contains $x$. Then $I(x)$ will be equal to the ideal generated by $M(x)$ over $A$; if $J \subset A$ is an ideal such that $x \in J \otimes_{\mathbb{Z}_{p}} Q$, then $M(x) \subset J$ and so $I(x) \subset J$.

We use that $\otimes_{\mathbb{Z}_{p}} Q$ commutes with finite intersections since $Q$ is $\mathbb{Z}_{p}$-flat. Choose a finitely generated $\mathbb{Z}_{p}$-submodule $M \subset A$ with $x \in M \otimes_{\mathbb{Z}_{p}} Q$. Since $p^{n} A=0, M$ has finite length and so contains only finitely many $\mathbb{Z}_{p}$-submodules. Thus, if $M(x)$ is the intersection of all $M^{\prime} \subset M$ with $x \in M^{\prime} \otimes_{\mathbb{Z}_{p}} Q$, then $x \in M(x) \otimes_{\mathbb{Z}_{p}} Q$. If $M^{\prime \prime} \subset A$ is any other $\mathbb{Z}_{p}$-submodule with $x \in M^{\prime \prime} \otimes_{\mathbb{Z}_{p}} Q$, then $x \in\left(M^{\prime \prime} \cap M\right) \otimes_{\mathbb{Z}_{p}} Q$ and so $M(x) \subset\left(M^{\prime \prime} \cap M\right) \subset M^{\prime \prime}$. Therefore $M(x)$ is as desired. 
In the proof of the following lemma, we use that both $\left[\pi^{\mathrm{b}}\right]$ and $\varphi^{-1}(\mu)$ are units in $W\left(C^{b}\right)$. This can be seen by observing that modulo $p$ both are nonzero in $C^{b}$.

LEMMA 2.2.8. Let $B$ be an A-algebra and $\mathfrak{M}_{B} \in \mathcal{L}^{\leqslant h}\left(V_{B}\right)$. There exists a unique ideal $I \subset B$ such that, for any A-algebra homomorphism $B \rightarrow B^{\prime}, \mathfrak{M}_{B} \otimes_{B} B^{\prime} \in$ $\mathcal{L}_{\text {crys }}^{\leqslant h}\left(V_{B^{\prime}}\right)$ if and only if $B \rightarrow B^{\prime}$ factors through $B \rightarrow B / I$.

Proof. Consideration of Teichmuller expansions shows that if $x \in W\left(C^{b}\right)$ and $p x \in A_{\text {inf }}$, then $x \in A_{\text {inf }}$. Therefore the $\mathfrak{S}$-module $Q:=W\left(C^{b}\right) / A_{\text {inf }}$ is $\mathbb{Z}_{p}$-flat, and so

$$
0 \rightarrow B^{\prime} \otimes_{\mathbb{Z}_{p}} A_{\text {inf }} \rightarrow B^{\prime} \otimes_{\mathbb{Z}_{p}} W\left(C^{b}\right) \rightarrow B^{\prime} \otimes_{\mathbb{Z}_{p}} Q \rightarrow 0
$$

is exact. Since $\mathfrak{M}_{B^{\prime}}:=\mathfrak{M}_{B} \otimes_{B} B^{\prime}$ is finite projective over $\mathfrak{S}_{B^{\prime}}$, applying $\mathfrak{M}_{B^{\prime}} \otimes_{\mathfrak{S}_{B^{\prime}}}$ to the above exact sequence yields a sequence

$$
0 \rightarrow \mathfrak{M}_{B^{\prime}} \otimes_{\mathfrak{S}} A_{\text {inf }} \rightarrow \mathfrak{M}_{B^{\prime}} \otimes_{\mathfrak{S}} W\left(C^{b}\right) \rightarrow \mathfrak{M}_{B^{\prime}} \otimes_{\mathfrak{S}} Q \rightarrow 0,
$$

which is again exact. Thus $\mathfrak{M}_{B^{\prime}} \in \mathcal{L}_{\text {crys }}^{\leqslant h}\left(V_{B^{\prime}}\right)$ if and only if, for every $m \in \mathfrak{M}_{B^{\prime}}$ and every $\sigma \in G_{K}$, the image of

$$
\frac{(\sigma-1)(m)}{\left[\pi^{b}\right] \varphi^{-1}(\mu)} \in \mathfrak{M}_{B^{\prime}} \otimes_{\mathfrak{S}} W\left(C^{b}\right)
$$

in $\mathfrak{M}_{B^{\prime}} \otimes_{\mathfrak{S}} Q$ is zero. In fact, since $\mathfrak{M}_{B^{\prime}}$ is generated over $B^{\prime}$ by the image of $\mathfrak{M}_{B}$, we need only consider $m$ contained in the image of $\mathfrak{M}_{B} \rightarrow \mathfrak{M}_{B^{\prime}}$.

As $\mathfrak{M}_{B}$ is finite projective over $\mathfrak{S}_{B}$, there is an isomorphism $\mathfrak{M}_{B} \oplus Z \cong$ $\left(B \otimes_{\mathbb{Z}_{p}} \mathfrak{S}\right)^{r}$ for some $\mathfrak{S}_{B}$-module $Z$. Thus we obtain an inclusion $\mathfrak{M}_{B} \otimes_{\mathfrak{S}} Q \hookrightarrow$ $\left(B \otimes_{\mathbb{Z}_{p}} Q\right)^{r}$. If $e_{i}$ denotes the standard basis of $\left(B \otimes_{\mathbb{Z}_{p}} Q\right)^{r}$ then, for every $m \in \mathfrak{M}_{B}$ and $\sigma \in G_{K}$, the image of $\left((\sigma-1)(m) /\left[\pi^{b}\right] \varphi^{-1}(\mu)\right)$ under $\mathfrak{M}_{B} \otimes_{\mathfrak{S}} W\left(C^{b}\right) \rightarrow$ $\mathfrak{M}_{B} \otimes_{\mathfrak{S}} Q \hookrightarrow\left(B \otimes_{\mathbb{Z}_{p}} Q\right)^{r}$ can be written as

$$
\sum \alpha(m, \sigma, i) e_{i}
$$

for some $\alpha(m, \sigma, i) \in B \otimes_{\mathbb{Z}_{p}} Q$. Let $I(m, \sigma, i) \subset B$ be the smallest ideal such that $\alpha(m, \sigma, i) \in I(m, \sigma, i) \otimes_{\mathbb{Z}_{p}} Q$ (which exists by Lemma 2.2.7) and let $I=\sum_{m, \sigma, i} I(m, \sigma, i)$. The discussion from the previous paragraph shows that $I$ is as required by the lemma.

Proposition 2.2.9. There exists a closed A-subscheme $\mathcal{L}_{A \text {, crys }}^{\leqslant h}$ of $\mathcal{L}_{A}^{\leqslant h}$, which represents the functor $B \mapsto \mathcal{L} \leqslant h\left(V_{B}\right)$.

Proof. To any morphism Spec $B \rightarrow \mathcal{L}^{\leqslant h}$ of $A$-schemes, we associate $\mathfrak{M}_{B} \in$ $\mathcal{L}^{\leqslant h}\left(V_{B}\right)$ and so an ideal $I_{B} \subset B$ as in Lemma 2.2.8. The uniqueness in 
Lemma 2.2.8 implies that if $B \rightarrow B^{\prime}$ is an $A$-algebra homomorphism, then $I_{B^{\prime}}$ is the ideal of $B^{\prime}$ generated by the image of $I_{B}$. Thus the association $B \mapsto I_{B}$ defines a coherent sheaf of ideals on $\mathcal{L}^{\leqslant h}$. Let $\mathcal{L}_{A \text {,crys }}^{\leqslant h}$ be the corresponding closed $A$-subscheme of $\mathcal{L}^{\leqslant h}$. Since a morphism Spec $B \rightarrow \mathcal{L}^{\leqslant h}$ of $A$-schemes factors through $\mathcal{L}_{A \text {,crys }}^{\leqslant h}$ if and only if $I_{B}=0$, and this occurs if and only if $\mathfrak{M}_{B} \in \mathcal{L}_{\text {crys }}^{\leqslant h}\left(V_{B}\right)$, it follows that $\mathcal{L}_{A \text {, crys }}^{\leqslant h}$ represents $B \mapsto \mathcal{L}_{\text {crys }}^{\leqslant h}\left(V_{B}\right)$.

2.2.10. Now let $A$ be a complete local Noetherian ring with residue field $\mathbb{F}$ and maximal ideal $\mathfrak{m}_{A}$. Let $V_{A}$ be a finite free $A$-module equipped with a continuous action of $G_{K}$.

COROLLARY 2.2.11. There exists a projective A-scheme $\mathcal{L}_{A \text {,crys, }}^{\leqslant h}$ which, for each $i \geqslant 1$, represents the functor $B \mapsto \mathcal{L}_{\text {crys }}^{\leqslant h}\left(V_{A} \otimes_{A} B\right)$ on A-algebras $B$ with $\mathfrak{m}_{A}^{i} B=0$.

Proof. Set $A_{i}=A / \mathfrak{m}_{A}^{i}$. The projective schemes $\mathcal{L}_{A_{i}, \text { crys }}^{\leqslant h}$ form an inverse system of schemes over $A_{i}$, and so a formal scheme over $A$. The very ample line bundles on each $\mathcal{L}_{A_{i}}^{\leqslant h}$ restrict to an inverse system of very ample line bundles on the $\mathcal{L}_{A_{i} \text {, crys }}^{\leqslant h}$. As a consequence of [18, Théorème 5.4.5], this formal scheme arises from a projective $A$ scheme as required.

2.2.12. Suppose $C$ is a local finite flat $\mathbb{Z}_{p}$-algebra and $V_{C}$ is a finite free $C$-module equipped with a continuous $C$-linear action of $G_{K}$. Then there is an $\mathcal{O}_{\mathcal{E}, C}=\mathcal{O}_{\mathcal{E}} \otimes_{\mathbb{Z}_{p}} C$-module $M_{C}$ equipped with an isomorphism $\varphi^{*} M_{C} \rightarrow M_{C}$ and a $\varphi, G_{K_{\infty}}$-equivariant identification $M_{C} \otimes_{\mathcal{O}_{\mathcal{E}}} W\left(C^{\mathrm{b}}\right) \cong V_{C} \otimes_{\mathbb{Z}_{p}} W\left(C^{\mathrm{b}}\right)$. In the obvious way, we make sense of the sets $\mathcal{L}^{\leqslant h}\left(V_{C}\right)$ and $\mathcal{L}_{\text {crys }}^{\leqslant h}\left(V_{C}\right)$. Thus $\mathfrak{M}_{C} \in \mathcal{L}^{\leqslant h}\left(V_{C}\right)$ if $\mathfrak{M}_{C} \subset M_{C}$ is a $\varphi$-stable projective $\mathfrak{S}_{C}=\mathfrak{S} \otimes_{\mathbb{Z}_{p}} C$-module so that $\mathfrak{M}_{C} \otimes_{\mathfrak{S}} \mathcal{O}_{\mathcal{E}}=M_{C}$ and so that $\varphi^{*} \mathfrak{M}_{C} \rightarrow \mathfrak{M}_{C}$ has cokernel is killed by $E(u)^{h}$. Further, $\mathfrak{M}_{C} \in \mathcal{L}_{\text {crys }}^{\leqslant h}\left(V_{C}\right)$ if the $G_{K}$-action on $\mathfrak{M}_{C} \otimes_{\mathfrak{S}} W\left(C^{b}\right) \cong V_{C} \otimes_{\mathbb{Z}_{p}} W\left(C^{\mathrm{b}}\right)$ is such that

$$
(\sigma-1)(m) \in \mathfrak{M}_{C} \otimes_{\mathfrak{S}}\left[\pi^{\mathrm{b}}\right] \varphi^{-1}(\mu) A_{\text {inf }}
$$

for all $\sigma \in G_{K}$ and $m \in \mathfrak{M}_{C}$.

2.2.13. Let $C$ be an $A$-algebra that is finite flat over $\mathbb{Z}_{p}$. A morphism Spec $C \rightarrow$ $\mathcal{L}_{A \text {, crys }}^{\leqslant h}$ gives morphisms $\mathcal{L}_{A \text {,crys }}^{\leqslant h} \rightarrow \operatorname{Spec} C_{i}$, where $C_{i}=C / p^{i} C$. For any $i \geqslant 1$, there is a $j$ such that $\mathfrak{m}_{A}^{j} C \subset p^{i} C$, and so, by Corollary 2.2.11, such a system of morphisms gives rise to $\mathfrak{M}_{C_{i}} \in \mathcal{L}_{\text {crys }}^{\leqslant h}\left(V_{C_{i}}\right)$ with $\mathfrak{M}_{C_{i}}=\mathfrak{M}_{C_{i+1}} \otimes_{C_{i+1}} C_{i}$. The limit $\mathfrak{M}_{C}=\lim _{\longleftarrow} \mathfrak{M}_{C_{i}}$ is a projective $\mathfrak{S}_{C}$-submodule of $M_{C}=\lim _{\longleftarrow} M_{C_{i}}$ defining an 
element of $\mathcal{L}^{\leqslant h}\left(V_{C}\right)$. Under the identification

$$
\mathfrak{M}_{C} \otimes_{\mathfrak{S}} W\left(C^{\mathrm{b}}\right)=V_{C} \otimes_{\mathbb{Z}_{p}} W\left(C^{\mathrm{b}}\right),
$$

the $G_{K}$ action on $\mathfrak{M}_{C} \otimes_{\mathfrak{S}} W\left(C^{b}\right)$ is such that, for each $i \geqslant 1$ and each $m \in \mathfrak{M}_{C}$, $\sigma \in G_{K}$, the images of the elements

$$
\frac{(\sigma-1)(m)}{\left[\pi^{b}\right] \varphi^{-1}(\mu)}
$$

in $\mathfrak{M}_{C_{i}} \otimes_{\mathfrak{S}} W\left(C^{b}\right)$ are contained in $\mathfrak{M}_{C_{i}} \otimes_{\mathfrak{S}} A_{\text {inf. }}$. Since $C$ is finite free as a $\mathbb{Z}_{p}$-module $\mathfrak{M}_{C}$ is projective, and hence free, over $\mathfrak{S}$. This implies these elements are contained in $\mathfrak{M}_{C} \otimes_{\mathfrak{S}} A_{\text {inf }}$ : since $\mathfrak{M}_{C}$ is free over $\mathfrak{S}$, it suffices to show that if $x \in W\left(C^{b}\right)$ is congruent to an element of $A_{\text {inf }}$ modulo $p^{i}$ for every $i \geqslant 1$, then $x \in A_{\text {inf }}$. Considering the Teichmuller expansion of $x$ shows this statement holds. Conversely, any $\mathfrak{M}_{C} \in \mathcal{L}_{\text {crys }}^{\leqslant h}\left(V_{C}\right)$ gives rise to a unique $C$-point of $\mathcal{L}_{A, \text { crys }}^{\leqslant h}$.

LEMMA 2.2.14. The morphism $\mathcal{L}_{A \text {,crys }}^{\leqslant h} \rightarrow$ Spec $A$ becomes a closed immersion after inverting $p$.

Proof. One argues exactly as in [21, 1.6.4]. As explained in loc. cit., any point of $\mathcal{L}=\mathcal{L}_{A, \text { crys }}^{\leqslant h}$ valued in a finite local $\mathbb{Q}_{p}$-algebra $B$ is induced from a $C$-valued point for a finite flat $\mathbb{Z}_{p}$-algebra $C \subset B$. We claim this implies $\mathcal{L}(B) \rightarrow(\operatorname{Spec} A)(B)$ is injective. Indeed, given two $B$-valued points of $\mathcal{L}$ inducing the same $B$-valued point of Spec $A$, the above produces a finite flat $\mathbb{Z}_{p}$-algebra $C \subset B$ so that both $B$-valued points factor through $\operatorname{Spec} B \rightarrow \operatorname{Spec} C$. The last sentence of 2.1.4 implies $\mathcal{L}_{\text {crys }}^{\leqslant h}\left(V_{C}\right)$ consists of at most one element, and so 2.2.13 implies both $B$-valued points of $\mathcal{L}$ are induced from the same $C$-valued point.

Taking $B=E$ for any finite extension $E / \mathbb{Q}_{p}$ shows that the proper morphism $\mathcal{L}_{A, \text { crys }}^{\leqslant h} \otimes_{\mathbb{Z}_{p}} \mathbb{Q}_{p} \rightarrow$ Spec $A[1 / p]$ is injective on closed points, and at these closed points induces an isomorphism of residue fields. Taking $B=E[\epsilon] /\left(\epsilon^{2}\right)$ shows that at these closed points this morphism also induces an injection of tangent spaces. We conclude it is a closed immersion.

Proposition 2.2.15. Let $A_{\text {crys }}^{\leqslant h}$ denote the quotient of A corresponding to the scheme-theoretic image of $\mathcal{L}_{A, \text { crys }}^{\leqslant h} \rightarrow$ Spec A. Then, we have the following:

(1) The morphism $\mathcal{L}_{A \text {,crys }}^{\leqslant h} \rightarrow$ Spec $A_{\text {crys }}^{\leqslant h}$ becomes an isomorphism after inverting $p$.

(2) For any finite $\mathbb{Q}_{p}$-algebra $B$, a map $A \rightarrow B$ factors through $A_{\text {crys }}^{\leqslant h}$ if and only if $V_{B}=V_{A} \otimes_{A} B$ is crystalline with Hodge-Tate weights contained in $[0, h]$. 
Proof. Part (1) follows from Lemma 2.2.14. As a consequence, as $B$ is a finite $\mathbb{Q}_{p}$-algebra, a map $A \rightarrow B$ factors through $A_{\text {crys }}^{\leqslant h}$ if and only if $A \rightarrow B$ is induced from a $B$-valued point of $\mathcal{L}_{A \text {, crys }}^{\leqslant h}$.

In proving (2), we may assume $B$ is local. As in the proof of Lemma 2.2.14, any $B$-valued point of $\mathcal{L}_{A \text {,crys }}^{\leqslant h}$ is induced from a $C$-valued point with $C$ finite flat over $\mathbb{Z}_{p}$. This in turn gives rise to an $\mathfrak{M}_{C} \in \mathcal{L}_{\text {crys }}^{\leqslant h}\left(V_{C}\right)$. The fact that the $G_{K}$-action on $\mathfrak{M}_{C} \otimes_{\mathbb{Z}_{p}} W\left(C^{b}\right)$ satisfies our usual condition implies, after Theorem 2.1.12, that $V_{C}=V_{A} \otimes_{A} C$ is a $\mathbb{Z}_{p}$-lattice inside the crystalline representation $V_{C}[1 / p]$ whose Hodge-Tate weights are contained in $[0, h]$. Thus the same is true for $V_{B}=V_{C}[1 / p] \otimes_{C[1 / p]} B$.

For the converse, suppose $A \rightarrow B$ is such that $V_{B}=V_{A} \otimes_{A} B$ is crystalline with Hodge-Tate weights contained in $[0, h]$. Then there is a finite flat $\mathbb{Z}_{p}$-algebra $C \subset B$ so that $A \rightarrow B$ factors through $C$. As $V_{C} \otimes_{\mathbb{Z}_{p}} \mathbb{Q}_{p}$ is a $G_{K}$-stable $\mathbb{Q}_{p}$-subspace of $V_{B}, V_{C} \otimes_{\mathbb{Z}_{p}} \mathbb{Q}_{p}$ is also crystalline with Hodge-Tate weights contained in $[0, h]$. Theorem 2.1.12 implies there exist a Breuil-Kisin module $\mathfrak{M}_{C}$ and a $\varphi, G_{K_{\infty}}$-equivariant identification

$$
\mathfrak{M}_{C} \otimes_{\mathfrak{S}} W\left(C^{b}\right) \cong V_{C} \otimes_{\mathbb{Z}_{p}} W\left(C^{b}\right)
$$

such that $(\sigma-1)(m) \in \mathfrak{M}_{C} \otimes_{\mathfrak{S}}\left[\pi^{\mathrm{b}}\right] \varphi^{-1}(\mu) A_{\text {inf }}$ for every $\sigma \in G_{K}$ and every $m \in \mathfrak{M}_{C}$. By functoriality, $\mathfrak{M}_{C}$ is an $\mathfrak{S}_{C}$-module, but it need not be projective. However, in the penultimate paragraph of the proof of $[21,1.6 .4]$ it is shown that, at the cost of enlarging $C$, one can arrange that $\mathfrak{M}_{C}$ is projective over $\mathfrak{S}_{C}$. Thus $A \rightarrow B$ arises from a $C$-point of $\mathcal{L}$ for some $C \subset B$ finite flat over $\mathbb{Z}_{p}$, and therefore from a $B$-point of $\mathcal{L}$. We conclude that $A \rightarrow B$ factors through $A_{\text {crys }}^{\leqslant h}$.

REMARK 2.2.16. (1) The fact that $\mathfrak{M}_{C}$ need not be $\mathfrak{S}_{C}$-projective, even though $V_{C}$ is projective as a $C$-module is related to the fact that the functor from finite $E$-height representations to Breuil-Kisin modules is not exact.

(2) There is one instance in which $V_{C}$ being $C$-projective implies $\mathfrak{M}_{C}$ is $\mathfrak{S}_{C}$-projective. This is when $C$ is the ring of integers of a finite extension of $\mathbb{Q}_{p}$. See for example [16, Proposition 3.4] for a proof. In particular, if $E / \mathbb{Q}_{p}$ is finite and $V$ is a $G_{K}$-stable $\mathcal{O}_{E}$-lattice inside a crystalline representation of $G_{K}$, then the Breuil-Kisin module associated with $V$ is an element of $\mathcal{L}_{\text {crys }}^{\leqslant p}(V)$.

\section{Strong divisibility}

For the rest of the paper, we assume $K$ is an unramified extension of $\mathbb{Q}_{p}$. 


\subsection{Strong divisibility}

3.1.1. Let $\mathbb{F}$ be a finite field of characteristic $p$ and $V_{\mathbb{F}}$ a finite free $\mathbb{F}$-module equipped with a continuous $\mathbb{F}$-linear action of $G_{K_{\infty}}$.

Definition 3.1.2. Let $\mathcal{L}_{\mathrm{SD}}^{\leqslant p}\left(V_{\mathbb{F}}\right)$ denote the set of $\mathfrak{M} \in \mathcal{L} \leqslant p\left(V_{\mathbb{F}}\right)$ for which there exist a $k[[u]]$-basis $\left(m_{i}\right)$ of $\mathfrak{M}$ and integers $r_{i}$ such that $\left(u^{r_{i}} m_{i}\right)$ forms a $k\left[\left[u^{p}\right]\right]$-basis of $\varphi(\mathfrak{M})$. We call $\mathfrak{M}$ satisfying this condition strongly divisible.

We are going to relate $\mathcal{L}_{\mathrm{SD}}^{\leqslant p}\left(V_{\mathbb{F}}\right)$ with $\mathcal{L}_{\text {crys }}^{\leqslant p}\left(V_{\mathbb{F}}\right)$. (Note that the latter set only makes sense when the $G_{K_{\infty}}$-action on $V_{\mathbb{F}}$ extends to a continuous $G_{K}$-action.) Before doing so, we record how some basic operations on $V_{\mathbb{F}}$ respect $\mathcal{L}_{\mathrm{SD}}^{\leqslant p}\left(V_{\mathbb{F}}\right)$ and $\mathcal{L}_{\text {crys }}^{\leqslant p}\left(V_{\mathbb{F}}\right)$.

LEMMA 3.1.3. Let $V_{\mathbb{F}}$ be as above and suppose $W_{\mathbb{F}}$ is another continuous representation of $G_{K_{\infty}}$ on an $\mathbb{F}$-vector space. Suppose $\mathfrak{M} \in \mathcal{L}_{\mathrm{SD}}^{\leqslant p}\left(V_{\mathbb{F}}\right)$ and $\mathfrak{N} \in \mathcal{L}^{\leqslant p}\left(W_{\mathbb{F}}\right)$.

(1) Suppose there exists a surjective $\varphi$-equivariant map of $k[[u]]$-modules $f: \mathfrak{M} \rightarrow \mathfrak{N}$. Then $\mathfrak{N} \in \mathcal{L}_{\mathrm{SD}}^{\leq p}\left(W_{\mathbb{F}}\right)$.

(2) Suppose there exists an injective $\varphi$-equivariant map of $k[[u]]-$ modules $f: \mathfrak{N} \rightarrow \mathfrak{M}$ with u-torsion-free cokernel. Then $\mathfrak{N} \in \mathcal{L}_{\mathrm{SD}}^{\leqslant p}\left(W_{\mathbb{F}}\right)$.

Proof. This follows from part (1) of [2, 5.4.6].

LEMMA 3.1.4. For any finite extension $\mathbb{F}^{\prime}$ of $\mathbb{F}$, the rule $\mathfrak{M} \mapsto \mathfrak{M} \otimes_{\mathbb{F}} \mathbb{F}^{\prime}$ defines a map

$$
\mathcal{L}^{\leqslant h}\left(V_{\mathbb{F}}\right) \rightarrow \mathcal{L}^{\leqslant h}\left(V_{\mathbb{F}} \otimes_{\mathbb{F}} \mathbb{F}^{\prime}\right) .
$$

Further, $\mathfrak{M} \in \mathcal{L}_{\mathrm{SD}}^{\leqslant p}\left(V_{\mathbb{F}}\right)$ if and only if its image lies in $\mathcal{L}_{\mathrm{SD}}^{\leqslant p}\left(V_{\mathbb{F}} \bigotimes_{\mathbb{F}} \mathbb{F}^{\prime}\right)$. If $V_{\mathbb{F}}$ admits a $G_{K}$-action, then likewise $\mathfrak{M} \in \mathcal{L}_{\text {crys }}^{\leqslant h}\left(V_{\mathbb{F}}\right)$ if and only if its image lies in $\mathcal{L}_{\text {crys }}^{\leqslant h}\left(V_{\mathbb{F}} \otimes_{\mathbb{F}} \mathbb{F}\right)$.

Proof. The only part that does not follow immediately from the definitions is that $\mathfrak{M} \otimes_{\mathbb{F}} \mathbb{F}^{\prime} \in \mathcal{L}_{\mathrm{SD}}^{\leqslant p}\left(V_{\mathbb{F}} \otimes_{\mathbb{F}} \mathbb{F}^{\prime}\right)$ implies $\mathfrak{M} \in \mathcal{L}_{\mathrm{SD}}^{\leqslant p}\left(V_{\mathbb{F}}\right)$. For this, note that the inclusion $\mathfrak{M} \rightarrow \mathfrak{M} \otimes_{\mathbb{F}} \mathbb{F}^{\prime}$ is $\varphi$-equivariant with $u$-torsion-free cokernel. Thus we can apply (2) of Lemma 3.1.3.

LEMMA 3.1.5. For any unramified $\mathbb{F}$-valued character $\psi$ of $G_{K}$, there is a bijection

$$
\mathcal{L}^{\leqslant h}\left(V_{\mathbb{F}}\right) \stackrel{\sim}{\rightarrow} \mathcal{L}^{\leqslant h}\left(V_{\mathbb{F}} \otimes_{\mathbb{F}} \mathbb{F}(\psi)\right),
$$


which identifies $\mathcal{L}_{\mathrm{SD}}^{\leqslant p}\left(V_{\mathbb{F}}\right)$ with $\mathcal{L}_{\mathrm{SD}}^{\leqslant p}\left(V_{\mathbb{F}} \otimes_{\mathbb{F}} \mathbb{F}(\psi)\right)$ and, if $V_{\mathbb{F}}$ admits a $G_{K}$-action, identifies $\mathcal{L}_{\text {crys }}^{\leqslant h}\left(V_{\mathbb{F}}\right)$ with $\mathcal{L}_{\text {crys }}^{\leqslant h}\left(V_{\mathbb{F}} \otimes_{\mathbb{F}} \mathbb{F}(\psi)\right)$.

Proof. First, we note there exists a $y \in\left(\bar{k} \bigotimes_{\mathbb{F}_{p}} \mathbb{F}\right)^{\times}$such that $\sigma(y)=\psi(\sigma)^{-1} y$ for all $\sigma \in G_{K}$, due to the assumption $\psi$ is unramified. Using $y$, we can describe the etale $\varphi$-module associated with $V_{\mathbb{F}}(\psi):=V_{\mathbb{F}} \bigotimes_{\mathbb{F}} \mathbb{F}(\psi)$ in terms of that associated with $V_{\mathbb{F}}$. To do this, consider the $\mathbb{F}$-linear map $V_{\mathbb{F}} \rightarrow V_{\mathbb{F}}(\psi)$ given by $v \mapsto v \otimes 1$. Applying $\otimes_{\mathbb{Z}_{p}} W\left(C^{b}\right)$ induces an identification

$$
V_{\mathbb{F}} \otimes_{\mathbb{Z}_{p}} W\left(C^{b}\right) \stackrel{\sim}{\rightarrow} V_{\mathbb{F}}(\psi) \otimes_{\mathbb{Z}_{p}} W\left(C^{b}\right),
$$

which is $\varphi$-equivariant when both sides are equipped with the Frobenius that is trivial on $V_{\mathbb{F}}$ and $V_{\mathbb{F}}(\psi)$. If $M \subset V_{\mathbb{F}} \otimes_{\mathbb{Z}_{p}} W\left(C^{b}\right)$ is the etale $\varphi$-module associated with $V_{\mathbb{F}}$, then its image $M(\psi)$ under this map is a finitely generated $\mathcal{O}_{\mathcal{E}}$-submodule of $\left(V_{\mathbb{F}} \otimes_{\mathbb{F}} \mathbb{F}(\psi)\right) \otimes_{\mathbb{Z}_{p}} W\left(C^{b}\right)$ on which Frobenius acts by an isomorphism and on which $G_{K_{\infty}}$ acts on by the character $\psi$. Via the inclusion $\bar{k} \rightarrow C^{b}$, we can view $y$ as an element of $C^{b} \otimes_{\mathbb{F}_{p}} \mathbb{F}$ and so may consider $y M(\psi) \subset V_{\mathbb{F}}(\psi) \otimes_{\mathbb{Z}_{p}} W\left(C^{b}\right)$. The $G_{K_{\infty}}$-action on $y M(\psi)$ is then trivial and, since

$$
\varphi(y) / y \in\left(k \otimes_{\mathbb{F}_{p}} \mathbb{F}\right)^{\times} \subset \mathfrak{S}_{\mathbb{F}}^{\times},
$$

the Frobenius on $y M(\psi)$ is still an isomorphism. Hence $y M(\psi)$ equals the etale $\varphi$-module associated with $V_{\mathbb{F}}(\psi)$.

We can then describe a map $\mathcal{L}^{\leqslant h}\left(V_{\mathbb{F}}\right) \rightarrow \mathcal{L}^{\leqslant h}\left(V_{\mathbb{F}}(\psi)\right)$ by sending $\mathfrak{M} \subset M$ onto $y \mathfrak{M}(\psi) \subset y M(\psi)$, where $\mathfrak{M}(\psi)$ equals the image of $\mathfrak{M}$ under (3.1.6). Clearly, this is a bijection. When $h=p$, it also clearly identifies $\mathcal{L}_{\mathrm{SD}}^{\leqslant p}\left(V_{\mathbb{F}}\right)$ and $\mathcal{L}_{\mathrm{SD}}^{\leqslant p}\left(V_{\mathbb{F}}(\psi)\right)$. Finally, if $V_{\mathbb{F}}$ admits a $G_{K}$-action and $\mathfrak{M} \in \mathcal{L}^{\leqslant h}\left(V_{\mathbb{F}}\right)$, then the $G_{K}$-action on $y \mathfrak{M}(\psi) \otimes_{\mathfrak{S}} W\left(C^{b}\right)$ identifies with the $G_{K}$-action on $\mathfrak{M} \otimes_{\mathfrak{S}} W\left(C^{b}\right)$ twisted by $\psi$. It follows that $\mathcal{L}_{\text {crys }}^{\leqslant h}\left(V_{\mathbb{F}}\right)$ and $\mathcal{L}_{\text {crys }}^{\leqslant h}\left(V_{\mathbb{F}}(\psi)\right)$ are also identified.

3.1.7. Note that for any $\mathbb{F}$-algebra $B$, the argument above shows that, for any finite free $B$-module $V_{B}$ equipped with a continuous $G_{K}$-action, there are functorial bijections

$$
\mathcal{L}_{\text {crys }}^{\leqslant h}\left(V_{B}\right) \cong \mathcal{L}_{\text {crys }}^{\leqslant h}\left(V_{B} \otimes_{B} B(\psi)\right) \quad \text { and } \quad \mathcal{L}^{\leqslant h}\left(V_{B}\right) \cong \mathcal{L}^{\leqslant h}\left(V_{B} \otimes_{B} B(\psi)\right) .
$$

Finally let $L / K$ be an unramified extension corresponding to a finite extension $l / k$ of residue fields. Set $L_{\infty}=L K_{\infty}$. If $\mathfrak{S}_{L}:=W(l)[[u]]$ is embedded into $W\left(C^{b}\right)$ as with $\mathfrak{S}$, by mapping $u$ onto $\left[\pi^{b}\right]$, then we can make sense of $\mathcal{L}^{\leqslant h}\left(\left.V_{\mathbb{F}}\right|_{G_{\infty}}\right)$ as in Definition 2.2.2, replacing $K$ and $K_{\infty}$ by $L$ and $L_{\infty}$. Its elements are modules over $\mathfrak{S}_{L, \mathbb{F}}=\mathfrak{S}_{L} \otimes_{\mathbb{Z}_{p}} \mathbb{F}$. We write $f: \mathfrak{S} \rightarrow \mathfrak{S}_{L}$ for the inclusion induced by the inclusion $k \subset l$. 
LEMMA 3.1.8. (1) There is a map

$$
f^{*}: \mathcal{L}^{\leqslant h}\left(V_{\mathbb{F}}\right) \rightarrow \mathcal{L}^{\leqslant h}\left(\left.V_{\mathbb{F}}\right|_{G_{\infty}}\right)
$$

sending $\mathfrak{M} \in \mathcal{L}^{\leqslant h}\left(V_{\mathbb{F}}\right)$ onto $\mathfrak{M} \otimes_{\mathfrak{S}, f} \mathfrak{S}_{L}$. If $\mathfrak{M} \in \mathcal{L}^{\leqslant p}\left(V_{\mathbb{F}}\right)$, then $\mathfrak{M} \in \mathcal{L}_{\mathrm{SD}}^{\leqslant p}\left(V_{\mathbb{F}}\right)$ if and only if $f^{*} \mathfrak{M}=\mathfrak{M} \otimes_{\mathfrak{S}} \mathfrak{S}_{L} \in \mathcal{L}_{\mathrm{SD}}^{\leqslant p}\left(\left.V_{\mathbb{F}}\right|_{G_{L_{\infty}}}\right)$. If $V_{\mathbb{F}}$ admits a $G_{K}$-action, then this map sends $\mathcal{L}_{\text {crys }}^{\leqslant h}\left(V_{\mathbb{F}}\right)$ into $\mathcal{L}_{\text {crys }}^{\leqslant h}\left(\left.V_{\mathbb{F}}\right|_{G_{L}}\right)$.

(2) If $V_{\mathbb{F}}$ is a $G_{L_{\infty}}$-representation, then restriction of scalars along $f$ (that is, by viewing an $\mathfrak{S}_{L}$-module $\mathfrak{M}$ as an $\mathfrak{S}$-module using $f: \mathfrak{S} \rightarrow \mathfrak{S}_{L}$ ) describes a map

$$
f_{*}: \mathcal{L}^{\leqslant h}\left(V_{\mathbb{F}}\right) \rightarrow \mathcal{L}^{\leqslant h}\left(\operatorname{Ind}_{G_{L_{\infty}}}^{G_{K_{\infty}}} V_{\mathbb{F}}\right) .
$$

Proof. The fact that there are maps $f^{*}$ and $f_{*}$ is explained in [2, 6.2.1 and 6.2.4]. The additional statements regarding the image of $f^{*}$ are all clear (for the observation that $\mathfrak{M} \otimes_{\mathfrak{S}} \mathfrak{S}_{L}$ is strongly divisible implies $\mathfrak{M}$ is strongly divisible, argue as in Lemma 3.1.4 by considering the inclusion $\mathfrak{M} \rightarrow \mathfrak{M} \otimes_{\mathfrak{S}} \mathfrak{S}_{L}$ whose cokernel is torsion-free).

\subsection{Strong divisibility in the irreducible case}

3.2.1. If $K^{\mathrm{t}}$ denotes the maximal tamely ramified extension of $K$ then, since $K_{\infty}$ is totally ramified over $K, K^{\mathrm{t}} \cap K_{\infty}=K$. Thus the restriction map from $\operatorname{Gal}\left(K_{\infty} K^{\mathrm{t}} / K_{\infty}\right)$ to the tame quotient $\mathrm{Gal}\left(K^{\mathrm{t}} / K\right)$ of $G_{K}$ is an isomorphism. As such, any tamely ramified $G_{K}$-representation is uniquely determined by its restriction to $G_{K_{\infty}}$ and conversely, any tamely ramified representation of $G_{K_{\infty}}$ (that is one which factors through $\operatorname{Gal}\left(\bar{K} / K_{\infty} K^{\mathrm{t}}\right)$ ) extends uniquely to a tame representation of $G_{K}$. In particular, this applies to irreducible representations of $G_{K}$ and $G_{K_{\infty}}$ on $\mathbb{F}$-vector spaces since both are tamely ramified.

Proposition 3.2.2. Suppose that $V_{\mathbb{F}}$ is irreducible as a $G_{K}$-representation. Then $\mathcal{L}_{\mathrm{SD}}^{\leqslant p}\left(V_{\mathbb{F}}\right)=\mathcal{L}_{\text {crys }}^{\leqslant p}\left(V_{\mathbb{F}}\right)$.

Before giving a proof, we make the following observation.

3.2.3. As we are working with $p$-torsion coefficients, the condition for $\mathfrak{M} \in \mathcal{L}^{\leqslant h}\left(V_{\mathbb{F}}\right)$ to lie in $\mathcal{L}_{\text {crys }}^{\leqslant h}\left(V_{\mathbb{F}}\right)$ can be simplified. The $v^{b}$-valuation of $\left[\pi^{\mathrm{b}}\right]$ modulo $p$ is $1 / e$ while the $v^{b}$-valuation of $\varphi^{-1}(\mu)$ modulo $p$ is $1 /(p-1)$. Thus $\mathfrak{M} \otimes_{\mathfrak{S}}\left[\pi^{b}\right] \varphi^{-1}(\mu) A_{\text {inf }}=\mathfrak{M} \otimes_{k[[u]]} I$, where $I \subset \mathcal{O}_{C^{b}}$ is the ideal $u^{1 / e+1 /(p-1)} \mathcal{O}_{C^{b}}$. As $K / \mathbb{Q}_{p}$ is assumed unramified, $e=1$ and so $\mathfrak{M} \in \mathcal{L}^{\leqslant h}\left(V_{\mathbb{F}}\right)$ is contained 
in $\mathcal{L}_{\text {crys }}^{\leqslant h}\left(V_{\mathbb{F}}\right)$ if and only if the induced $G_{K}$-action on $\mathfrak{M} \otimes_{\mathfrak{S}} W\left(C^{b}\right)=\mathfrak{M} \otimes_{k[[u]]} C^{b}$ is such that

$$
(\sigma-1)(m) \in \mathfrak{M} \otimes_{k[[u]]} u^{p /(p-1)} \mathcal{O}_{C^{b}}
$$

for every $\sigma \in G_{K}$ and $m \in \mathfrak{M}$.

Proof of Proposition 3.2.2. Using Lemma 3.1.4, we can assume $\mathbb{F}$ is sufficiently large so that $[2,2.1 .2]$ applies. Thus there is an unramified extension $L / K$ such that $V_{\mathbb{F}} \cong \operatorname{Ind}_{G_{L}}^{G_{K}} W_{\mathbb{F}}$ for a one-dimensional $G_{L}$-representation $W_{\mathbb{F}}$. In particular, $\left.\left.V_{\mathbb{F}}\right|_{G_{K_{\infty}}} \cong \operatorname{Ind}_{G_{L_{\infty}}}^{G_{K_{\infty}}} W_{\mathbb{F}}\right|_{G_{L_{\infty}}}$.

3.2.4. We claim that if $\mathfrak{M} \in \mathcal{L} \leqslant p\left(V_{\mathbb{F}}\right)$, then there exists an $\mathfrak{N} \in \mathcal{L} \leqslant p\left(W_{\mathbb{F}}\right)$ so that $\mathfrak{M} \subset f_{*} \mathfrak{N}$ with $\mathfrak{M}[1 / u]=\left(f_{*} \mathfrak{N}\right)[1 / u]$. This is essentially $[2,6.3 .1]$ except that in loc. cit., $\mathfrak{M}$ is assumed to be strongly divisible, an assumption that turns out to be unnecessary. To prove the claim, consider the map $\left.V_{\mathbb{F}}\right|_{G_{L \infty}} \rightarrow W_{\mathbb{F}}$ corresponding to $V_{\mathbb{F}} \cong \operatorname{Ind}_{G_{L}}^{G_{K}} W_{\mathbb{F}}$ under Frobenius reciprocity. Lemma 3.2.5 produces a $\varphi$-equivariant surjection $f^{*} \mathfrak{M} \rightarrow \mathfrak{N}$ for some $\mathfrak{N} \in \mathcal{L}^{\leqslant p}\left(W_{\mathbb{F}}\right)$. Via the usual adjunction between $f_{*}$ and $f^{*}$, we obtain a nonzero map

$$
\mathfrak{M} \rightarrow f_{*} f^{*} \mathfrak{M} \rightarrow f_{*} \mathfrak{N},
$$

which is easily checked to be $\varphi$-equivariant. This map must be injective since a nonzero kernel would induce a nonzero $G_{K_{\infty}}$-subspace of $V_{\mathbb{F}}$. It must be an isomorphism after inverting $u$ because both $\mathfrak{M}$ and $f_{*} \mathfrak{N}$ have the same rank as $k[[u]]$-modules.

LEMMA 3.2.5. Let $0 \rightarrow W_{\mathbb{F}} \rightarrow V_{\mathbb{F}} \rightarrow Z_{\mathbb{F}} \rightarrow 0$ be a $G_{K_{\infty}}$-equivariant exact sequence. If $\mathfrak{M} \in \mathcal{L}^{\leqslant h}\left(V_{\mathbb{F}}\right)$, then there exist $\mathfrak{W} \in \mathcal{L}^{\leqslant h}\left(W_{\mathbb{F}}\right)$ and $\mathfrak{Z} \in \mathcal{L}^{\leqslant h}\left(Z_{\mathbb{F}}\right)$ together with $\varphi$-equivariant exact sequence

$$
0 \rightarrow \mathfrak{W} \rightarrow \mathfrak{M} \rightarrow \mathfrak{Z} \rightarrow 0
$$

which identifies with $0 \rightarrow W_{\mathbb{F}} \rightarrow V_{\mathbb{F}} \rightarrow Z_{\mathbb{F}} \rightarrow 0$ after base-changing to $W\left(C^{b}\right)$.

Proof. Since the equivalence between $G_{K_{\infty}}$-representations and etale $\varphi$-modules is exact, there is a $\varphi$-equivariant exact sequence $0 \rightarrow N_{\mathbb{F}} \rightarrow M_{\mathbb{F}} \rightarrow P_{\mathbb{F}} \rightarrow 0$ of etale $\varphi$-modules, which identifies with $0 \rightarrow W_{\mathbb{F}} \rightarrow V_{\mathbb{F}} \rightarrow Z_{\mathbb{F}} \rightarrow 0$ after base change to $W\left(C^{b}\right)$. Take $\mathfrak{W}=\mathfrak{M} \cap N_{\mathbb{F}}$ and $\mathfrak{Z}=\operatorname{Im}(\mathfrak{M}) \subset P_{\mathbb{F}}$. It is clear that both are $\varphi$-stable projective $\mathfrak{S}_{\mathbb{F}}$-modules. It is also clear that $u^{h} \mathfrak{Z} \subset \mathfrak{Z}^{\varphi}$ since the same is true of $\mathfrak{M}$, and so $\mathfrak{Z} \in \mathcal{L}^{\leqslant h}\left(Z_{\mathbb{F}}\right)$. Since $\mathfrak{Z}$ is $u$-torsion-free, $u^{h} \mathfrak{W}=u^{h} \mathfrak{M} \cap N_{\mathbb{F}}$. As $\mathfrak{W}^{\varphi}=\mathfrak{M}^{\varphi} \cap N_{\mathbb{F}}$, we conclude $u^{h} \mathfrak{W} \subset \mathfrak{M}^{\varphi}$. 
3.2.6. Return to the proof of Proposition 3.2.2 and fix $\mathfrak{N} \in \mathcal{L}^{\leqslant p}\left(W_{\mathbb{F}}\right)$ as in 3.2.4. Since $W_{\mathbb{F}}$ is one-dimensional, we can describe $\mathfrak{N}$ explicitly. We may suppose that $l$, the residue field of $L$, admits an embedding into $\mathbb{F}$. In this case, $\mathfrak{S}_{L, \mathbb{F}}=l[[u]] \otimes_{\mathbb{F}_{p}} \mathbb{F}=\prod_{\theta \in \operatorname{Hom}_{\mathbb{F} p}(l, \mathbb{F})} \mathbb{F}[[u]]$, where the identification is such that $l$ acts on the $\theta$ th component of the product through $\theta: l \rightarrow \mathbb{F}$. Viewing $\mathfrak{N}$ as an $\mathbb{F}[[u]]$-module via the diagonal embedding into $\mathfrak{S}_{L, \mathbb{F}}$, it follows from $[2,6.1 .1]$ that $\mathfrak{N}$ admits an $\mathbb{F}[[u]]$-basis $\left(e_{\theta}\right)_{\theta \in \operatorname{Hom}_{\mathbb{F}}}(l, \mathbb{F})$ satisfying

$$
\varphi\left(e_{\theta \circ \varphi}\right)=x u^{r_{\theta}} e_{\theta}
$$

for some $x \in l \otimes_{\mathbb{F}_{p}} \mathbb{F}$ and integers $r_{\theta} \geqslant 0$. Since $\mathfrak{N} \in \mathcal{L} \leqslant p\left(W_{\mathbb{F}}\right)$, we have $r_{\theta} \in[0, p]$. This basis is chosen so that $l$ acts on $e_{\theta}$ through $\theta$.

3.2.8. By twisting $V_{\mathbb{F}}$, and so $W_{\mathbb{F}}$, by an unramified character, which is harmless by Lemma 3.1.5, we may assume that $x$ in (3.2.7) equals 1. Under this assumption, $[2,6.5 .1]$ says that a finite free $\mathfrak{S}_{\mathbb{F}}$-submodule $\mathfrak{M} \subset f_{*} \mathfrak{N}$ satisfying $\mathfrak{M}[1 / u]=$ $\left(f_{*} \mathfrak{N}\right)[1 / u]$ is an element of $\mathcal{L}_{\mathrm{SD}}^{\leqslant p}\left(V_{\mathbb{F}}\right)$ if and only if:

(1) If $m \in \mathfrak{M}$ then $\varphi(m) \in \mathfrak{M}$, and if $\varphi(m) \in u^{p+1} \mathfrak{M}$ then $m \in u \mathfrak{M}$.

(2) For every $\mathbb{F}$-linear combination $\sum \alpha_{\theta} e_{\theta}$ which is contained in $\mathfrak{M}$, and every $0<r \leqslant p$, the $\mathbb{F}$-linear combination

$$
\sum_{r_{\theta} \equiv r \bmod p} \alpha_{\theta} e_{\theta}
$$

is contained in $\mathfrak{M}$ also.

Observe that $u^{p} \mathfrak{M} \subset \mathfrak{M}^{\varphi} \subset \mathfrak{M}$ implies (1). Indeed, if $\varphi(m) \in u^{p+1} \mathfrak{M}$, then $\varphi(m) \in u \mathfrak{M}^{\varphi}$. If $e_{i}$ is a $k[[u]]$-basis of $\mathfrak{M}$, then $m=\sum \alpha_{i} e_{i}$ for $\alpha_{i} \in k[[u]]$ and $\varphi(m)=\sum \varphi\left(\alpha_{i}\right) \varphi\left(e_{i}\right)$; by definition, the $\varphi\left(e_{i}\right)$ form a $k[[u]]$-basis of $\mathfrak{M}^{\varphi}$, so if $\varphi(m) \in u \mathfrak{M}^{\varphi}$ we must have each $\varphi\left(\alpha_{i}\right)$ divisible by $u$. This implies each $\alpha_{i}$ is also divisible by $u$.

Another consequence of (1) is that $u e_{\theta} \in \mathfrak{M}$ for every $\theta \in \operatorname{Hom}_{\mathbb{F}_{p}}(l, \mathbb{F})$. This is explained in the second paragraph after $[2,6.5 .1]$.

3.2.9. To finish the proof, we have to show that if $\mathfrak{M} \in \mathcal{L} \leqslant p\left(V_{\mathbb{F}}\right)$ is contained in $f_{*} \mathfrak{N}$, then (2) is satisfied if and only if $\mathfrak{M} \in \mathcal{L}_{\text {crys }}^{\leqslant p}\left(V_{\mathbb{F}}\right)$. The $G_{K}$-action on $V_{\mathbb{F}}$ induces a continuous $C^{b} \otimes_{\mathbb{F}_{p}} \mathbb{F}$-semilinear $\varphi$-equivariant action of $G_{K}$ on $V_{\mathbb{F}} \otimes_{\mathbb{F}_{p}} C^{b}=\mathfrak{M} \otimes_{k[[u]]} C^{b}$. Conversely, any such semilinear $G_{K}$-action induces a $G_{K}$-action on $V_{\mathbb{F}}$ extending the $G_{K_{\infty}}$-action. Thus 3.2.1 implies there is at most one such semilinear $G_{K}$-action. This semilinear action of $G_{K}$ can be written explicitly as follows:

$$
\sigma\left(e_{\theta}\right)=\eta(\sigma)^{\Theta_{\theta}} e_{\theta}, \quad \sigma \in G_{K},
$$


where $\Theta_{\theta}=\sum_{i=0}^{\left[l: \mathbb{F}_{p}\right]-1} p^{i} r_{\theta \circ \varphi^{i}}$ and $\eta(\sigma) \in \mathcal{O}_{C^{b}}$ is the unique $\left(p^{\left[l: \mathbb{F}_{p}\right]}-1\right)$ th root of $\sigma(u) / u$ whose image in the residue field of $\mathcal{O}_{C^{b}}$ is 1 . To verify this, it suffices to check this does indeed define a group action, that this action is continuous, that it induces the trivial action of $G_{K_{\infty}}$ on $\left(f_{*} \mathfrak{N}\right)[1 / u]=\mathfrak{M}[1 / u]$, and is $\varphi$-equivariant. The first three are straightforward to check, and checking the $\varphi$-equivariance comes down to the identity

$$
\sigma\left(u^{r_{\theta}}\right) \eta(\sigma)^{\Theta_{\theta}}=u^{r_{\theta}} \eta(\sigma)^{p \Theta_{\theta \circ \varphi}},
$$

which follows since

$$
p \Theta_{\theta \circ \varphi}=\sum_{i=0}^{\left[l: \mathbb{F}_{p}\right]-1} p^{i+1} r_{\theta \circ \varphi^{i}}=\left(p^{\left[l: \mathbb{F}_{p}\right]-1}-1\right) r_{\theta}+\sum_{i=0}^{\left[l: \mathbb{F}_{p}\right]-1} p^{i} r_{\theta \circ \varphi^{i}}
$$

Therefore this must be the $G_{K}$-action coming from that on $V_{\mathbb{F}}$. To check the condition from 3.2.3, we shall need the following lemma.

LeMma 3.2.11. For $\sigma \in G_{K}$, let $m=m(\sigma)$ be such that $\sigma(u) / u \in \mathbb{Z}_{p}(1)$ is a $\mathbb{Z}_{p}$-generator of $p^{m} \mathbb{Z}_{p}(1)$. Then, for $n \geqslant 0$,

$$
v^{b}\left(\eta(\sigma)^{n}-1\right)=\frac{p^{1+m+v_{p}(n)}}{p-1} .
$$

Proof. This easily reduces to the well-known calculation that $v^{b}(\epsilon-1)=$ $p /(p-1)$ for any $\mathbb{Z}_{p}$-generator $\epsilon \in \mathbb{Z}_{p}(1)$; see for example [10, Section 5.1.2].

We have to show that (2) is equivalent to asking that $(\sigma-1)(m) \in \mathfrak{M} \otimes_{k[u]]}$ $u^{p / p-1} \mathcal{O}_{C^{b}}$ for every $m \in \mathfrak{M}$ and $\sigma \in G_{K}$ (see 3.2.3). When $m=u^{i} e_{\theta}$ for $i \geqslant 1$, this follows easily from Lemma 3.2.11 since

$$
(\sigma-1)\left(u^{i} e_{\theta}\right)=\left(\left(\frac{\sigma(u)}{u}\right)^{i} \eta(\sigma)^{\Theta_{\theta}}-1\right)\left(u^{i} e_{\theta}\right)=\left(\eta(\sigma)^{\Theta_{\theta}+\left(p^{\left[l: \mathbb{F}_{p}\right]}-1\right) i}-1\right)\left(u^{i} e_{\theta}\right) .
$$

To complete the proof, we consider elements $\sum \alpha_{\theta} e_{\theta} \in \mathfrak{M}$ with $\alpha_{\theta} \in \mathbb{F}$. We compute that

$$
\begin{aligned}
\frac{(\sigma-1)\left(\sum \alpha_{\theta} e_{\theta}\right)}{\eta(\sigma)-1} & =\sum\left(\frac{\eta(\sigma)^{\Theta}-1}{\eta(\sigma)-1}\right) \alpha_{\theta} e_{\theta} \\
& =\sum\left(1+\eta(\sigma)+\cdots \eta(\sigma)^{\Theta_{\theta}-1}\right) \alpha_{\theta} e_{\theta} \\
& =\sum r_{\theta} \alpha_{\theta} e_{\theta}+\sum \beta_{\theta} u e_{\theta} \text { for some } \beta_{\theta} \in \mathcal{O}_{C^{b}}
\end{aligned}
$$


The last equality follows because $\eta(\sigma)-1 \in u^{p / p-1} \mathcal{O}_{C^{b}}$ by Lemma 3.2.11, and so $1+\eta(\sigma)+\cdots+\eta(\sigma)^{\Theta_{\theta}-1} \equiv \Theta_{\theta} \equiv r_{\theta}$ modulo $u^{p / p-1} \mathcal{O}_{C^{b}}$. Since $u e_{\theta} \in \mathfrak{M}$ for every $\theta$, it follows that $\left((\sigma-1)\left(\sum \alpha_{\theta} e_{\theta}\right)\right) /(\eta(\sigma)-1) \in \mathfrak{M} \otimes_{k[u]]} \mathcal{O}_{C^{b}}$ if and only if

$$
\sum r_{\theta} \alpha_{\theta} e_{\theta} \in \mathfrak{M}
$$

Since $v^{b}(\eta(\sigma)-1) \geqslant p /(p-1)$, with equality when $\sigma$ is chosen so that $\sigma(u) / u$ is a $\mathbb{Z}_{p}$-generator of $\mathbb{Z}_{p}(1)$, we conclude that $(\sigma-1)\left(\sum \alpha_{\theta} e_{\theta}\right) \in \mathfrak{M} \otimes_{k[[u]]} u^{p / p-1} \mathcal{O}_{C^{b}}$ for every $m \in \mathfrak{M}$ and $\sigma \in G_{K}$ if and only if for every $\mathbb{F}$-linear combination $\sum \alpha_{\theta} e_{\theta} \in \mathfrak{M}$, we have $\sum r_{\theta} \alpha_{\theta} e_{\theta} \in \mathfrak{M}$. It is easy to check the latter condition is equivalent to 2 .

\subsection{Strong divisibility in general}

Proposition 3.3.1. Suppose $V_{\mathbb{F}}$ admits a continuous $\mathbb{F}$-linear $G_{K}$-action. Then $\mathcal{L}_{\text {crys }}^{\leqslant p}\left(V_{\mathbb{F}}\right) \subset \mathcal{L}_{\mathrm{SD}}^{\leqslant p}\left(V_{\mathbb{F}}\right)$. (Unlike in the irreducible case, this inclusion is not always an equality. The problem arises from the possibility that $V_{\mathbb{F}}$ may admit two different $G_{K}$-actions extending a given $G_{K_{\infty}}$-action. Here is an example. Suppose $V_{\mathbb{F}}$ admits an $\mathbb{F}$-basis $\left(f_{1}, f_{2}\right)$ so that

$$
\sigma\left(f_{1}, f_{2}\right)=\left(f_{1}, f_{2}\right)\left(\begin{array}{cc}
1 & c(\sigma) \\
0 & \chi_{\text {cyc }}^{-1}(\sigma)
\end{array}\right)
$$

for a 1-cocycle c $(\sigma)$. We compute that

$$
\sigma\left(f_{1}, u^{1 / p-1} f_{2}\right)=\left(f_{1}, u^{1 / p-1} f_{2}\right)\left(\begin{array}{cc}
1 & \sigma\left(u^{1 / p-1}\right) c(\sigma) \\
0 & \frac{\sigma\left(u^{1 / p-1}\right)}{u^{1 / p-1}} \chi_{\mathrm{cyc}}^{-1}(\sigma)
\end{array}\right) .
$$

There exists cocycle $c$ such that $c(\sigma)=0$ for $\sigma \in G_{K_{\infty}}$; this occurs when $V_{\mathbb{F}}$ is a tres ramifie extension (see $[17,5.4 .2])$. In this case, the matrix representing $\sigma$ on $\left(f_{1}, u^{1 / p-1} f_{2}\right)$ is the identity when $\sigma \in G_{K_{\infty}}$, so $\mathfrak{M}$, the $\mathfrak{S}_{\mathbb{F}}$-span of $f_{1}$ and $u^{1 / p-1} f_{2}$, is contained in the etale $\varphi$-module associated with $V_{\mathbb{F}}$. Since

$$
\varphi\left(f_{1}, u^{1 / p-1} f_{2}\right)=\left(f_{1}, u^{1 / p-1} f_{2}\right)\left(\begin{array}{ll}
1 & 0 \\
0 & u
\end{array}\right),
$$

it is easy to see that $\mathfrak{M} \in \mathcal{L}_{\mathrm{SD}}^{\leqslant 1}\left(V_{\mathbb{F}}\right)$. However, $\mathfrak{M} \notin \mathcal{L}_{\text {crys }}^{\leqslant 1}\left(V_{\mathbb{F}}\right)$ since $u^{1 / p-1} c(\sigma)$ is not contained in $u^{p / p-1} \mathcal{O}_{C^{b}}$. The point is that $V_{\mathbb{F}}$ does not arise as the reduction modulo $p$ of a crystalline representation with Hodge-Tate weights in $[0,1]$.

To prove this, we will need to understand how $\mathcal{L}_{\mathrm{SD}}^{\leqslant p}$ and $\mathcal{L}_{\text {crys }}^{\leqslant p}$ behave in short exact sequences. 
3.3.2. Let $0 \rightarrow W_{\mathbb{F}} \rightarrow V_{\mathbb{F}} \rightarrow Z_{\mathbb{F}} \rightarrow 0$ be a $G_{K_{\infty}}$-equivariant exact sequence and let $\mathfrak{M} \in \mathcal{L}^{\leqslant p}\left(V_{\mathbb{F}}\right)$. Lemma 3.2.5 provides a $\varphi$-equivariant exact sequence

$$
0 \rightarrow \mathfrak{W} \rightarrow \mathfrak{M} \rightarrow \mathfrak{Z} \rightarrow 0
$$

with $\mathfrak{W} \in \mathcal{L}^{\leqslant h}\left(W_{\mathbb{F}}\right)$ and $\mathfrak{Z} \in \mathcal{L}^{\leqslant h}\left(Z_{\mathbb{F}}\right)$. Choosing an $\mathfrak{S}_{\mathbb{F}}$-splitting of (3.3.3) allows us to identify $\mathfrak{M}=\mathfrak{W} \oplus \mathfrak{Z}$ as $\mathfrak{S}_{\mathbb{F}}$-modules so that

$$
\varphi_{\mathfrak{M}}=\left(\varphi_{\mathfrak{W}}+f \circ \varphi_{\mathfrak{Z}}, \varphi_{\mathfrak{Z}}\right)
$$

for some $f \in \operatorname{Hom}(\mathfrak{Z}, \mathfrak{W})[1 / u]$. Here $\operatorname{Hom}(\mathfrak{Z}, \mathfrak{W})$ denotes the module of $\mathfrak{S}_{\mathbb{F}}$-linear homomorphisms $\mathfrak{Z} \rightarrow \mathfrak{W}$. Since $\mathfrak{M}^{\varphi} \subset \mathfrak{M}$, we must have $f\left(\mathfrak{Z}^{\varphi}\right) \subset \mathfrak{W}$ and so, as $u^{p} \mathfrak{Z} \subset \mathfrak{Z}^{\varphi}$, it follows that $f \in\left(1 / u^{p}\right) \operatorname{Hom}(\mathfrak{Z}, \mathfrak{W})$.

We equip $\operatorname{Hom}(\mathfrak{Z}, \mathfrak{W})$ with the Frobenius $\varphi$ given by $\varphi(g)=\varphi_{\mathfrak{W}} \circ g \circ \varphi_{\mathfrak{Z}}^{-1}$. Since any two splittings of (3.3.3) differ by an element $g \in \operatorname{Hom}(\mathfrak{Z}, \mathfrak{W})$, by choosing a different splitting, we replace $f$ by $f+(\varphi-1)(g)$. If as usual $\operatorname{Hom}(\mathfrak{Z}, \mathfrak{W})^{\varphi}$ denotes the $\mathfrak{S}_{\mathbb{F}}$-submodule of $\operatorname{Hom}(\mathfrak{Z}, \mathfrak{W})[1 / E]$ generated by $\varphi(\operatorname{Hom}(\mathfrak{Z}, \mathfrak{W}))$, then

$$
u^{p} \operatorname{Hom}(\mathfrak{Z}, \mathfrak{W}) \subset \operatorname{Hom}(\mathfrak{Z}, \mathfrak{W})^{\varphi} \subset \frac{1}{u^{p}} \operatorname{Hom}(\mathfrak{Z}, \mathfrak{W}) .
$$

Furthermore, we can $G_{K}$-equivariantly identify $\left(\operatorname{Hom}(\mathfrak{Z}, \mathfrak{W}) \otimes_{k[[u]]} C^{b}\right)^{\varphi=1}=$ $\operatorname{Hom}\left(Z_{\mathbb{F}}, W_{\mathbb{F}}\right)$ (the $G_{K}$-action on $\operatorname{Hom}\left(Z_{\mathbb{F}}, W_{\mathbb{F}}\right)$ being given by $f \mapsto \sigma \circ f \circ \sigma^{-1}$ ) via the identifications $\left(\mathfrak{Z} \otimes_{k[[u]]} C^{b}\right)^{\varphi=1}=Z_{\mathbb{F}}$ and $\left(\mathfrak{W} \otimes_{k[[u]]} C^{b}\right)^{\varphi=1}=W_{\mathbb{F}}$.

Proposition 3.3.5. In the situation of 3.3.2:

(1) If $\mathfrak{M} \in \mathcal{L}_{\mathrm{SD}}^{\leqslant p}\left(V_{\mathbb{F}}\right)$, then $\mathfrak{W} \in \mathcal{L}_{\mathrm{SD}}^{\leqslant p}\left(W_{\mathbb{F}}\right)$ and $\mathfrak{Z} \in \mathcal{L}_{\mathrm{SD}}^{\leqslant p}\left(Z_{\mathbb{F}}\right)$.

(2) If $\mathfrak{W} \in \mathcal{L}_{\mathrm{SD}}^{\leqslant p}\left(W_{\mathbb{F}}\right)$ and $\mathfrak{Z} \in \mathcal{L}_{\mathrm{SD}}^{\leqslant p}\left(Z_{\mathbb{F}}\right)$, then $\mathfrak{M} \in \mathcal{L}_{\mathrm{SD}}^{\leqslant p}\left(V_{\mathbb{F}}\right)$ if and only if there exists $g \in \operatorname{Hom}(\mathfrak{Z}, \mathfrak{W})$ such that

$$
f+(\varphi-1)(g) \in \operatorname{Hom}(\mathfrak{Z}, \mathfrak{W}) .
$$

Proof. Part (1) is a consequence of Lemma 3.1.3, while (2) follows from [3, Lemma 4.1.3].

3.3.6. Now suppose $0 \rightarrow W_{\mathbb{F}} \rightarrow V_{\mathbb{F}} \rightarrow Z_{\mathbb{F}} \rightarrow 0$ is an exact sequence of $G_{K}$-representations. As in 3.3.2, for any $\mathfrak{M} \in \mathcal{L} \leqslant p\left(V_{\mathbb{F}}\right)$, there is an exact sequence $0 \rightarrow \mathfrak{W} \rightarrow \mathfrak{M} \rightarrow \mathfrak{Z} \rightarrow 0$ so that, after choosing a splitting of this sequence and identifying $\mathfrak{M}=\mathfrak{W} \oplus \mathfrak{Z}$, we have $\varphi_{\mathfrak{M}}=\left(\varphi_{\mathfrak{W}}+f \circ \varphi_{\mathfrak{Z}}, \varphi_{\mathfrak{Z}}\right)$ for some $f \in\left(1 / u^{p}\right) \operatorname{Hom}(\mathfrak{Z}, \mathfrak{W})$.

As $0 \rightarrow W_{\mathbb{F}} \rightarrow V_{\mathbb{F}} \rightarrow Z_{\mathbb{F}} \rightarrow 0$ and $0 \rightarrow \mathfrak{W} \rightarrow \mathfrak{M} \rightarrow \mathfrak{Z} \rightarrow 0$ become identified after applying $\otimes_{k[[u]]} C^{b}$, we obtain compatible $\varphi$-equivariant $G_{K}$-actions on 
$\mathfrak{M} \otimes_{k[[u]]} C^{b}, \mathfrak{W}^{b} \otimes_{k[[u]]} C^{b}$ and $\mathfrak{Z}^{b} \otimes_{k[[u]]} C^{b}$. Under the identification $\mathfrak{M}=\mathfrak{W} \oplus \mathfrak{Z}$, the action of $\sigma \in G_{K}$ can be written as

$$
\sigma_{\mathfrak{M}}=\left(\sigma_{\mathfrak{W}}+f_{\sigma} \circ \sigma_{\mathfrak{Z}}, \sigma_{\mathfrak{Z}}\right)
$$

for some $f_{\sigma} \in \operatorname{Hom}(\mathfrak{Z}, \mathfrak{W}) \bigotimes_{k[u]]} C^{b}$ satisfying the following conditions:

(1) Since $\sigma_{\mathfrak{M}}$ is a group action, we must have $f_{\sigma \tau}=f_{\sigma}+\sigma_{\mathfrak{W}} \circ f_{\tau} \circ \sigma_{\mathfrak{Z}}^{-1}$. If we equip $\operatorname{Hom}(\mathfrak{Z}, \mathfrak{W}) \otimes_{k[[u]]} C^{b}$ with the $G_{K}$-action given by $\sigma(f)=$ $\sigma_{\mathfrak{W}} \circ f \circ \sigma_{\mathfrak{Z}}^{-1}$, then this says that $\sigma \mapsto f_{\sigma}$ is a 1-cocycle valued in $\operatorname{Hom}(\mathfrak{Z}$, $\mathfrak{W}) \otimes_{k[[u]]} C^{b}$. Since the $G_{K}$-action on $V_{\mathbb{F}}$ is continuous, $\sigma \mapsto f_{\sigma}$ must also be a continuous cocycle.

(2) Since the $G_{K_{\infty}}$-action on $V_{\mathbb{F}}$ is induced by the trivial action on $\mathfrak{M}$, we must have $\sigma_{\mathfrak{M}}(m)=m$ for every $m \in \mathfrak{M}$ and $\sigma \in G_{K_{\infty}}$. Thus we must have $f_{\sigma}(m)=0$ whenever $m \in \mathfrak{Z}$ and $\sigma \in G_{K_{\infty}}$.

(3) Since $\sigma_{\mathfrak{M}}$ is $\varphi$-equivariant, we must have $(\varphi-1)\left(f_{\sigma}\right)=(\sigma-1)(f)$ for any $\sigma \in G_{K}$.

Proposition 3.3.7. In the situation of 3.3.6:

(1) If $\mathfrak{M} \in \mathcal{L}_{\text {crys }}^{\leqslant p}\left(V_{\mathbb{F}}\right)$, then $\mathfrak{W} \in \mathcal{L}_{\text {crys }}^{\leqslant p}\left(W_{\mathbb{F}}\right)$ and $\mathfrak{Z} \in \mathcal{L}_{\text {crys }}^{\leqslant p}\left(Z_{\mathbb{F}}\right)$.

(2) If $\mathfrak{W} \in \mathcal{L}_{\text {crys }}^{\leqslant p}\left(W_{\mathbb{F}}\right)$ and $\mathfrak{Z} \in \mathcal{L}_{\text {crys }}^{\leqslant p}\left(Z_{\mathbb{F}}\right)$, then $\mathfrak{M} \in \mathcal{L}_{\text {crys }}^{\leqslant p}\left(V_{\mathbb{F}}\right)$ if and only if $f_{\sigma} \in \operatorname{Hom}(\mathfrak{Z}, \mathfrak{W}) \otimes_{k[[u]]} u^{p / p-1} \mathcal{O}_{C^{b}}$ for every $\sigma \in G_{K}$.

Proof. For the second statement, combine 3.2.3 with 3.3.6. For the first, as $0 \rightarrow$ $\mathfrak{W} \rightarrow \mathfrak{M} \rightarrow \mathfrak{Z} \rightarrow 0$ becomes $G_{K}$-equivariant after applying $\otimes_{\mathfrak{S}} W\left(C^{b}\right)$, it is clear that $(\sigma-1)(z) \in \mathfrak{Z} \otimes_{k[u]]} u^{p / p-1} \mathcal{O}_{C^{b}}$ for $z \in \mathfrak{Z}$. Thus $\mathfrak{Z} \in \mathcal{L}_{\text {crys }}^{\leqslant p}\left(V_{\mathbb{F}}\right)$. If $n \in \mathfrak{W}$, then $(\sigma-1)(n) \in \mathfrak{M} \otimes_{k[[u]]} u^{p / p-1} \mathcal{O}_{C^{b}} \cap \mathfrak{W} \otimes_{k[[u]]} C^{b}$; this intersection equals $\mathfrak{W} \otimes_{k[[u]]} u^{p / p-1} \mathcal{O}_{C^{b}}$ because $\mathfrak{Z}$ is $u$-torsion-free, and so $\mathfrak{W} \in \mathcal{L}_{\text {crys }}^{\leqslant p}\left(V_{\mathbb{F}}\right)$ also.

Proof of Proposition 3.3.1. Using Lemma 3.1.4, we can replace $\mathbb{F}$ by a finite extension. As explained in the beginning of the proof of Proposition 3.2.2, this allows us to assume each Jordan-Holder factor of $V_{\mathbb{F}}$ is induced from a onedimensional representation over an unramified extension of $K$. Using (1) of Lemma 3.1.8, we may then replace $K$ by a suitably large (but finite) unramified extension so that every Jordan-Holder factor of $V_{\mathbb{F}}$ is one-dimensional. Under this assumption, we argue by induction on the length (equivalently the dimension) of $V_{\mathbb{F}}$.

The base case of the induction is handled by Proposition 3.2.2. Thus we can assume $V_{\mathbb{F}}$ fits into a $G_{K}$-equivariant exact sequence $0 \rightarrow W_{\mathbb{F}} \rightarrow V_{\mathbb{F}} \rightarrow Z_{\mathbb{F}} \rightarrow 0$ with $Z_{\mathbb{F}}$ one-dimensional over $\mathbb{F}$ and $W_{\mathbb{F}} \neq 0$. 
As in 3.3.2, if $\mathfrak{M} \in \mathcal{L} \leqslant p\left(V_{\mathbb{F}}\right)$, we obtain an exact sequence $0 \rightarrow \mathfrak{W} \rightarrow \mathfrak{M} \rightarrow$ $\mathfrak{Z} \rightarrow 0$ with $\mathfrak{W} \in \mathcal{L}^{\leqslant p}\left(W_{\mathbb{F}}\right)$ and $\mathfrak{Z} \in \mathcal{L}^{\leqslant p}\left(Z_{\mathbb{F}}\right)$. By choosing a splitting of this sequence, we identify $\mathfrak{M}=\mathfrak{W} \oplus \mathfrak{Z}$ as $\mathfrak{S}_{\mathbb{F}}$-modules, with Frobenius given by

$$
\varphi_{\mathfrak{M}}=\left(\varphi_{\mathfrak{W}}+f \circ \varphi_{\mathfrak{Z}}, \varphi_{\mathfrak{Z}}\right)
$$

for some $f \in\left(1 / u^{p}\right) \operatorname{Hom}(\mathfrak{Z}, \mathfrak{W})$. As in 3.3.6, the $G_{K}$-action on $\mathfrak{M} \otimes_{k[u]]} C^{b}$ induced by the $G_{K}$-action on $V_{\mathbb{F}}$ may be written as

$$
\sigma_{\mathfrak{M}}=\left(\sigma_{\mathfrak{W}}+f_{\sigma} \circ \sigma_{\mathfrak{Z}}, \sigma_{\mathfrak{Z}}\right)
$$

for some $f_{\sigma} \in \operatorname{Hom}(\mathfrak{Z}, \mathfrak{W}) \bigotimes_{k[[u]]} C^{\text {b }}$ satisfying $(\varphi-1)\left(f_{\sigma}\right)=(\sigma-1)(f)$. If $\mathfrak{M} \in \mathcal{L}_{\text {crys }}^{\leqslant p}\left(V_{\mathbb{F}}\right)$, then $\mathfrak{W} \in \mathcal{L}_{\text {crys }}^{\leqslant p}\left(W_{\mathbb{F}}\right), \mathfrak{Z} \in \mathcal{L}_{\text {crys }}^{\leqslant p}\left(Z_{\mathbb{F}}\right)$, and

$$
f_{\sigma} \in \operatorname{Hom}(\mathfrak{Z}, \mathfrak{W}) \otimes_{k[[u]]} u^{p / p-1} \mathcal{O}_{C^{b}}
$$

by Proposition 3.3.7. By induction, $\mathfrak{W} \in \mathcal{L}_{\mathrm{SD}}^{\leqslant p}\left(W_{\mathbb{F}}\right)$ and $\mathfrak{Z} \in \mathcal{L}_{\mathrm{SD}}^{\leqslant p}\left(Z_{\mathbb{F}}\right)$. By Proposition 3.3.5, $\mathfrak{M} \in \mathcal{L}_{\mathrm{SD}}^{\leqslant p}\left(V_{\mathbb{F}}\right)$ if and only if

$$
f \in \operatorname{Hom}(\mathfrak{Z}, \mathfrak{W})+\varphi(\operatorname{Hom}(\mathfrak{Z}, \mathfrak{W})) .
$$

Using (3.3.4) and (3.3.8), we see $(\varphi-1)\left(f_{\sigma}\right)=(\sigma-1)(f) \in \operatorname{Hom}(\mathfrak{Z}, \mathfrak{W}) \bigotimes_{k[[u]]}$ $u^{p / p-1} \mathcal{O}_{C^{b}}$. Thus the proposition follows from the following claim.

Claim. Any $f \in\left(1 / u^{p}\right) \operatorname{Hom}(\mathfrak{Z}, \mathfrak{W})$ satisfying $(\sigma-1)(f) \in \operatorname{Hom}(\mathfrak{Z}, \mathfrak{W}) \bigotimes_{k[[u]]}$ $u^{p / p-1} \mathcal{O}_{C^{b}}$ must be contained in $\operatorname{Hom}(\mathfrak{Z}, \mathfrak{W})+\varphi(\operatorname{Hom}(\mathfrak{Z}, \mathfrak{W}))$.

Proof of claim. We argue by a further induction, this time on the length of $W_{\mathbb{F}}$. Recall that by assumption, every Jordan-Holder factor of $W_{\mathbb{F}}$ is one-dimensional. Thus the base case is when both $W_{\mathbb{F}}$ and $Z_{\mathbb{F}}$ are one-dimensional. In this case, as explained in 3.2.6, $\mathfrak{W}$ and $\mathfrak{Z}$, respectively, admit $\mathbb{F}[[u]]$-bases $\left(w_{\tau}\right)_{\tau \in \operatorname{Hom}_{\mathbb{F}}(k, \mathbb{F})}$ and $\left(z_{\tau}\right)_{\tau \in \operatorname{Hom}_{\mathbb{F}}(k, \mathbb{F})}$ so that

$$
\varphi\left(w_{\tau \circ \varphi}\right)=x u^{r_{\tau}} w_{\tau}, \quad \varphi\left(z_{\tau \circ \varphi}\right)=y u^{s_{\tau}} z_{\tau}
$$

for $x, y \in\left(k \otimes_{\mathbb{F}_{p}} \mathbb{F}\right)^{\times}$and $r_{\tau}, s_{\tau} \in[0, p]$. The $\mathbb{F}[[u]]$-linear homomorphism $F_{\tau}: \mathfrak{Z} \rightarrow \mathfrak{W}$ sending $z_{\tau^{\prime}} \mapsto 0$ for $\tau^{\prime} \neq \tau$ and $z_{\tau} \mapsto w_{\tau}$ is $\mathfrak{S}_{\mathbb{F}}$-linear since it is compatible with the $k$-action on $\mathfrak{Z}$ and $\mathfrak{W}$ (by construction, $k$-acts on $z_{\tau}$ and $w_{\tau}$ by $\tau$ ). Thus $F_{\tau} \in \operatorname{Hom}(\mathfrak{Z}, \mathfrak{W})$ and together the $F_{\tau}$ form an $\mathbb{F}[[u]]$-basis of $\operatorname{Hom}(\mathfrak{Z}, \mathfrak{W})$ satisfying $\varphi\left(F_{\tau \circ \varphi}\right)=x y^{-1} u^{t_{\tau}} F_{\tau}$ for $t_{\tau}=r_{\tau}-s_{\tau} \in[-p, p]$. Since the $G_{K}$-actions on $\mathfrak{Z} \otimes_{k[[u]]} C^{b}$ and $\mathfrak{W} \otimes_{k[[u]]} C^{b}$ are as in (3.2.10), we also have that

$$
\sigma\left(F_{\tau}\right)=\eta(\sigma)^{\Theta_{\tau}} F_{\tau}, \quad \Theta_{\tau}=\sum_{i=0}^{\left[k: \mathbb{F}_{p}\right]-1} t_{\tau \circ \varphi^{i}} p^{i}
$$


To prove the claim, it suffices to consider $f=\left(\sum_{0>i \geqslant-p} a_{i} u^{i}\right) F_{\tau}$. Then $(\sigma-1)(f)$ equals

$$
\sum_{0>i \geqslant-p} a_{i} u^{i}(\underbrace{\eta(\sigma)^{\Theta_{\tau}+\left(p^{[k: \mathbb{F} p]}-1\right) i}-1}_{(a)}) F_{\tau}
$$

(recall that $\eta(\sigma)$ is a $\left(p^{\left[k: \mathbb{F}_{p}\right]}-1\right)$ th root of $\left.\sigma(u) / u\right)$. Choose $\sigma$ so that $\sigma(u) / u$ is a $\mathbb{Z}_{p}$-generator of $\mathbb{Z}_{p}(1)$. Since

$$
\Theta_{\tau}+\left(p^{\left[k: \mathbb{F}_{p}\right]}-1\right) i \equiv t_{\tau}-i \text { modulo } p,
$$

Lemma 3.2.11 implies the $v^{b}$-valuation of $(a)$ is $p /(p-1)$ if $t_{\tau}-i$ is not divisible by $p$, and is $\geqslant p^{2} /(p-1)$ otherwise. Hence

$$
v^{b}\left(u^{i}\left(\eta(\sigma)^{\Theta_{\tau}+\left(p^{[k: \mathbb{F} p]}-1\right) i}\right)\right) \begin{cases}=p /(p-1)+i & \text { if } p \text { does not divide } t_{\tau}-i \\ \geqslant p^{2} /(p-1)+i & \text { otherwise. }\end{cases}
$$

Since $p^{2}(p-1)+i \geqslant p /(p-1)$ for $i \geqslant-p$ it follows that

$$
(\sigma-1)(f) \in \operatorname{Hom}(\mathfrak{Z}, \mathfrak{W}) \otimes_{k[[u]]} u^{p / p-1} \mathcal{O}_{C^{b}}
$$

if and only if $a_{i}=0$ except possibly if $i=t_{\tau}$; in other words, if and only if $f \in \operatorname{Hom}(\mathfrak{Z}, \mathfrak{W})+\varphi(\operatorname{Hom}(\mathfrak{Z}, \mathfrak{W}))$.

Now we prove the inductive step. Let $0 \rightarrow W_{\mathbb{F}}^{1} \rightarrow W_{\mathbb{F}} \rightarrow W_{\mathbb{F}}^{2} \rightarrow 0$ be an exact sequence of $G_{K}$-representations. As in 3.3.2 and 3.3.6, we can write $\mathfrak{W}=\mathfrak{W}^{1} \oplus \mathfrak{W}^{2}$ with $\mathfrak{W}^{i} \in \mathcal{L}_{\text {crys }}^{\leqslant p}\left(W_{\mathbb{F}}^{i}\right)$, so that $\varphi_{\mathfrak{W}}=\left(\varphi_{\mathfrak{W}^{1}}+g \circ \varphi_{\mathfrak{W}^{2}}, \varphi_{\mathfrak{W}^{2}}\right)$ for some $g \in \operatorname{Hom}\left(\mathfrak{W}^{2}, \mathfrak{W}^{1}\right)$, and so that $\sigma_{\mathfrak{W}}=\left(\sigma_{\mathfrak{W}^{1}}+g_{\sigma} \circ \sigma_{\mathfrak{W}^{2}}, \sigma_{\mathfrak{W}^{2}}\right)$ for some $g_{\sigma} \in \operatorname{Hom}\left(\mathfrak{W}^{2}, \mathfrak{W}^{1}\right) \otimes_{k[u]]} u^{p / p-1} \mathcal{O}_{C^{b}}$. Applying $\operatorname{Hom}(\mathfrak{Z},-)$, this allows us to identify $\mathfrak{H}:=\operatorname{Hom}(\mathfrak{Z}, \mathfrak{W})$ with $\mathfrak{H}^{1} \oplus \mathfrak{H}^{2}$, where $\mathfrak{H}^{i}=\operatorname{Hom}\left(\mathfrak{Z}, \mathfrak{W}^{i}\right)$, so that

$$
\varphi_{\mathfrak{H}}=\left(\varphi_{\mathfrak{H}^{1}}+\tilde{g} \circ \varphi_{\mathfrak{H}^{2}}, \varphi_{\mathfrak{H}^{2}}\right), \quad \sigma_{\mathfrak{H}}=\left(\sigma_{\mathfrak{H}^{1}}+\widetilde{g}_{\sigma} \circ \sigma_{\mathfrak{H}^{2}}, \sigma_{\mathfrak{H}^{2}}\right)
$$

where $\widetilde{g} \in \operatorname{Hom}\left(\mathfrak{H}^{2}, \mathfrak{H}^{1}\right)$ sends $h \mapsto g \circ h$ and where $\widetilde{g}_{\sigma} \in \operatorname{Hom}\left(\mathfrak{H}^{2}, \mathfrak{H}^{1}\right) \bigotimes_{k[[u]]}$ $u^{p / p-1} \mathcal{O}_{C^{b}}$ sends $h \mapsto g_{\sigma} \circ h$. If we write $f=\left(f_{1}, f_{2}\right) \in\left(1 / u^{p}\right)\left(\mathfrak{H}^{1} \oplus \mathfrak{H}^{2}\right)$ then, as $\left(\sigma_{\mathfrak{H}}-1\right)(f) \in \mathfrak{H} \otimes_{k[[u]]} u^{p / p-1} \mathcal{O}_{C^{b}}$, we have

$$
\begin{aligned}
\left(\sigma_{\mathfrak{H}^{2}}-1\right)\left(f_{2}\right) & \in \mathfrak{H}^{2} \otimes_{k[[u]]} u^{p / p-1} \mathcal{O}_{C^{b}} \\
\left(\sigma_{\mathfrak{H}^{1}}-1\right)\left(f_{1}\right)+\widetilde{g}_{\sigma} \circ \sigma_{\mathfrak{H}^{2}}\left(f_{2}\right) & \in \mathfrak{H}^{1} \otimes_{k[[u]]} u^{p / p-1} \mathcal{O}_{C^{b}} .
\end{aligned}
$$

By our inductive hypothesis, we deduce $f_{2}=f_{2}^{\prime}+f_{2}^{\prime \prime}$ with $f_{2}^{\prime} \in \mathfrak{H}^{2}$ and $f_{2}^{\prime \prime} \in$ $\varphi\left(\mathfrak{H}^{2}\right)$. Thus, we can write

$$
f=\underbrace{\left(f_{1}-\tilde{g}\left(f_{2}^{\prime}\right), f_{2}^{\prime}\right)}_{:=y}+\underbrace{\left(\widetilde{g}\left(f_{2}^{\prime \prime}\right), f_{2}^{\prime \prime}\right)}_{:=z}
$$


with $z \in \varphi(\mathfrak{H})$. Since $\varphi(\mathfrak{H}) \subset u^{-p} \mathfrak{H}$, we have $(\sigma-1)(z) \in \mathfrak{H} \otimes_{k[[u]]} u^{p / p-1} \mathcal{O}_{C^{b}}$. Thus $(\sigma-1)(y) \in \mathfrak{H} \otimes_{k[[u]]} u^{p / p-1} \mathcal{O}_{C^{b}}$. We also see $y \in u^{-p} \mathfrak{H}$. This means that to prove the result for $f$, it suffices to do so for $y$, that is, we can assume in the above that $f_{2}^{\prime \prime}=0$. Thus, $f_{2} \in \mathfrak{H}^{2}$ and so $\sigma_{\mathfrak{H}^{2}}\left(f_{2}\right) \in \mathfrak{H}^{2} \otimes_{k[u]]} \mathcal{O}_{C^{b}}$. Since

$$
\widetilde{g}_{\sigma} \in \operatorname{Hom}\left(\mathfrak{H}^{2}, \mathfrak{H}^{1}\right) \otimes_{k[[u]]} u^{p / p-1} \mathcal{O}_{C^{b}},
$$

it follows that $\widetilde{g}_{\sigma} \circ \sigma_{\mathfrak{H}^{2}}\left(f_{2}\right) \in \mathfrak{H}^{1} \bigotimes_{k[[u]]} u^{p / p-1} \mathcal{O}_{C^{b}}$. Thus $\left(\sigma_{\mathfrak{H}^{1}}-1\right)\left(f_{1}\right) \in \mathfrak{H}^{1} \bigotimes_{k[[u]]}$ $u^{p / p-1} \mathcal{O}_{C^{b}}$ and so $f_{1} \in \mathfrak{H}^{1}+\varphi\left(\mathfrak{H}^{1}\right)$ by induction also. We conclude that $f \in$ $\mathfrak{H}+\varphi\left(\mathfrak{H}^{1}\right) \subset \mathfrak{H}+\varphi(\mathfrak{H})$.

\section{Local structure of $\mathcal{L}_{\text {crys }}^{\leqslant p}$ in the unramified case}

\subsection{Commutative algebra}

Lemma 4.1.1. Let $A$ be a local Noetherian $\mathbb{Z}_{p}$-algebra with $A[1 / p] \neq 0$ and residue field of characteristic $p$.

(1) If $\mathfrak{p} \subset A[1 / p]$ is a maximal ideal and $\mathfrak{q}$ denotes its preimage in $A$, then $\operatorname{dim} A_{\mathfrak{q}} \leqslant \operatorname{dim} A-1$.

(2) If the residue field of $A$ is finite, then $A / \mathfrak{q}$ is finite over $\mathbb{Z}_{p}$ and the residue field of $A_{\mathfrak{q}}$ is finite over $\mathbb{Q}_{p}$.

Proof. The inclusion $A / \mathfrak{q} \rightarrow A[1 / p] / \mathfrak{p}$ becomes an isomorphism after inverting $p$, and so $\operatorname{dim} A / \mathfrak{q} \leqslant 1$ by [19, 10.5.1]. Since $A / \mathfrak{q}$ is a domain and not a field (its residue field has characteristic $p$ ), it must be that $\operatorname{dim} A / \mathfrak{q}=1$. Thus $\operatorname{dim} A_{\mathfrak{q}} \leqslant \operatorname{dim} A-1$. For (2), by the above, $A /(\mathfrak{q}, p)$ is zero-dimensional. Thus $A /(\mathfrak{q}, p)$ is an Artin local ring with finite residue field; so it is finite over $\mathbb{F}_{p}$. As such $A / \mathfrak{q}$ is finite over $\mathbb{Z}_{p}$ (see [28, Tag 031D]) and so $A[1 / p] / \mathfrak{p}=A_{\mathfrak{q}} / \mathfrak{q} A_{\mathfrak{q}}$ is $\mathbb{Q}_{p}$-finite.

LEMMA 4.1.2. Let A be a Noetherian local $\mathbb{Z}_{p}$-algebra with finite residue field. Suppose that $A$ is reduced, $\mathbb{Z}_{p}$-flat, and Nagata (see [28, Tag 032E]). If $\mathfrak{m}_{A}$ denotes the maximal ideal of $A$ and $j \geqslant 1$, then there exists a finite flat $\mathbb{Z}_{p}$-algebra $C$ such that $A \rightarrow A / \mathfrak{m}_{A}^{j}$ factors through a map $A \rightarrow C$.

Proof. If $A$ is $\mathbb{Z}_{p}$-flat, then so is its $\mathfrak{m}_{A}$-adic completion. If $A$ is Nagata and reduced, then its $\mathfrak{m}_{A}$-adic completion is reduced; see [28, Tag 07NZ]. Thus we may assume $A$ is $\mathfrak{m}_{A}$-adically complete.

For every maximal ideal $\mathfrak{p} \subset A[1 / p]$, Lemma 4.1 .1 shows that $A /(\mathfrak{p} \cap A)$ is finite flat over $\mathbb{Z}_{p}$. As $A[1 / p]$ is Jacobson (see [28, Tag 02IM]), the intersection of 
its maximal ideals equals its nilradical, and this is zero because $A$ is reduced and $\mathbb{Z}_{p}$-flat. Thus $\bigcap(\mathfrak{p} \cap A)=0$, the intersection running over all maximal ideals in $A[1 / p]$. The same is true if the intersection runs over a suitably chosen countable subset $\left\{\mathfrak{p}_{1}, \mathfrak{p}_{2}, \ldots\right\}$ of all maximal ideals in $A[1 / p]$. (The Artin-Rees lemma implies $\mathfrak{p}=\bigcap_{n \geqslant 1}\left(\mathfrak{p}+\mathfrak{m}_{A}^{n}\right)$ and so the intersection of the ideals in $\left\{\mathfrak{p}+\mathfrak{m}_{A}^{n}\right\}_{\mathfrak{p}, n}$, which consists of countably many distinct ideals, equals the intersection of the $\mathfrak{p}$. Thus there exists a countable subset $\left\{\mathfrak{p}_{i}\right\}_{i} \geqslant 1$ of the $\mathfrak{p}$ 's, and integers $n_{i} \geqslant 1$, such that $\bigcap \mathfrak{p}=\bigcap_{i}\left(\mathfrak{p}_{i}+\mathfrak{m}_{A}^{n_{i}}\right)$. Since $\mathfrak{p}_{i} \subset \mathfrak{p}_{i}+\mathfrak{m}_{A}^{n_{i}}$, we have $\bigcap \mathfrak{p}=\bigcap \mathfrak{p}_{i}$.) The $\mathfrak{q}_{i}=\bigcap_{i=1}^{i}\left(\mathfrak{p}_{i} \cap A\right)$ then form a decreasing sequence of closed ideals in $A$ whose intersection is zero. It follows from [6, III, Section 2, Proposition 8] that there exists an $n$ such that $\mathfrak{q}_{n} \subset \mathfrak{m}_{A}^{j}$. Setting $C=A / \mathfrak{q}_{n}$ proves the lemma.

\subsection{Hodge types and connected components}

4.2.1. Let $B$ be an arbitrary $\mathbb{Z}_{p}$-algebra and $\mathfrak{M}_{B}$ a finite projective $\mathfrak{S}_{B}$-module equipped with a map $\varphi^{*} \mathfrak{M}_{B} \rightarrow \mathfrak{M}_{B}$ with cokernel killed by $E(u)^{h}$. For any $B$-algebra $B^{\prime}$, set $\mathfrak{M}_{B^{\prime}}=\mathfrak{M}_{B} \otimes_{B} B^{\prime}$. For $i \geqslant 0$, define

$$
K^{i}\left(\mathfrak{M}_{B}\right)=\operatorname{coker}\left(\mathfrak{M}_{B}^{\varphi} \rightarrow \mathfrak{M}_{B} / E(u)^{i} \mathfrak{M}_{B}\right) .
$$

This $\mathfrak{S} \otimes_{\mathbb{Z}_{p}} B$-module is finite over $B$. On $\mathfrak{M}_{B}^{\varphi}$ we define a filtration

$$
F^{i}\left(\mathfrak{M}_{B}^{\varphi}\right)=\mathfrak{M}_{B}^{\varphi} \cap E(u)^{i} \mathfrak{M}_{B}
$$

with graded piece $\operatorname{gr}^{i}\left(\mathfrak{M}_{B}^{\varphi}\right)$. Note that multiplication by $E(u)$ induces an injection $\operatorname{gr}^{i-1}\left(\mathfrak{M}_{B}^{\varphi}\right) \rightarrow \operatorname{gr}^{i}\left(\mathfrak{M}_{B}^{\varphi}\right)$. We let $\mathcal{G}^{i}\left(\mathfrak{M}_{B}^{\varphi}\right)$ denote its cokernel. Thus $\mathcal{G}^{i}\left(\mathfrak{M}_{B}^{\varphi}\right)$ is the $i$ th graded piece of the filtered $\mathcal{O}_{K} \otimes_{\mathbb{Z}_{p}} B$-module $\mathfrak{M}_{B}^{\varphi} / E(u) \mathfrak{M}_{B}^{\varphi}$ whose $i$ th filtered piece is the image of $F^{i}\left(\mathfrak{M}_{B}^{\varphi}\right)$. It follows from (2) of Proposition 2.1.10 that, for $B$ a finite $\mathbb{Q}_{p}$-algebra,

$$
\mathcal{G}^{i}\left(\mathfrak{M}_{B}^{\varphi}\right)=\operatorname{gr}^{i}\left(D_{\mathrm{dR}}\left(V_{B}\right)\right)
$$

whenever $\mathfrak{M}_{B}$ is the Breuil-Kisin module associated with a crystalline representation $V_{B}$.

LEMMA 4.2.3. (1) $K^{i}\left(\mathfrak{M}_{B}\right)$ is $B$-flat if and only if $F^{i}\left(\mathfrak{M}_{B}^{\varphi}\right) \otimes_{B} B^{\prime} \rightarrow F^{i}\left(\mathfrak{M}_{B^{\prime}}^{\varphi}\right)$ is surjective for every quotient $B^{\prime}=B / I$.

(2) Each $\mathcal{G}^{i}\left(\mathfrak{M}_{B}^{\varphi}\right)$ is $\mathcal{O}_{K} \otimes_{\mathbb{Z}_{p}} B$-finite and for every $B$-algebra $B^{\prime}$, there are natural $\mathcal{O}_{K} \otimes_{\mathbb{Z}_{p}}$ B-module homomorphisms $\mathcal{G}^{i}\left(\mathfrak{M}_{B}^{\varphi}\right) \otimes_{B} B^{\prime} \rightarrow \mathcal{G}^{i}\left(\mathfrak{M}_{B^{\prime}}^{\varphi}\right)$.

(3) If (1) holds for all $i \geqslant 0$, then the maps in (2) are isomorphisms, and each $\mathcal{G}^{i}\left(\mathfrak{M}_{B}^{\varphi}\right)$ is B-flat. 
This lemma does not require $K$ to be unramified over $\mathbb{Q}_{p}$.

Proof. The kernel of $\mathfrak{M}_{B} / E(u)^{i} \mathfrak{M}_{B} \rightarrow K^{i}\left(\mathfrak{M}_{B}\right)$ is equal to $\mathfrak{M}_{B}^{\varphi} / F^{i}\left(\mathfrak{M}_{B}^{\varphi}\right)$. Thus $\mathfrak{M}_{B}^{\varphi} / F^{i}\left(\mathfrak{M}_{B}^{\varphi}\right)$ is $B$-finite. As $K^{i}\left(\mathfrak{M}_{B}\right)$ is of formation compatible with base change, being the cokernel of a map between modules compatible with base change, there are surjective maps

$$
\left(\mathfrak{M}_{B}^{\varphi} / F^{i}\left(\mathfrak{M}_{B}^{\varphi}\right)\right) \otimes_{B} B^{\prime} \rightarrow \mathfrak{M}_{B}^{\varphi} / F^{i}\left(\mathfrak{M}_{B^{\prime}}^{\varphi}\right)
$$

whose kernel is $\operatorname{Tor}_{1}^{B}\left(K^{i}\left(\mathfrak{M}_{B}\right), B^{\prime}\right)$. Since this kernel can be identified with the cokernel of $F^{i}\left(\mathfrak{M}_{B}^{\varphi}\right) \otimes_{B} B^{\prime} \rightarrow F^{i}\left(\mathfrak{M}_{B^{\prime}}^{\varphi}\right)$, we deduce 1 . Since $\operatorname{gr}^{i}\left(\mathfrak{M}_{B}^{\varphi}\right)$ is the kernel of the obvious surjection $\mathfrak{M}_{B}^{\varphi} / F^{i+1}\left(\mathfrak{M}_{B}^{\varphi}\right) \rightarrow \mathfrak{M}_{B}^{\varphi} / F^{i}\left(\mathfrak{M}_{B}^{\varphi}\right)$, we see each $\operatorname{gr}^{i}\left(\mathfrak{M}_{B}^{\varphi}\right)$ is $B$-finite. We also obtain maps

$$
\operatorname{gr}^{i}\left(\mathfrak{M}_{B}^{\varphi}\right) \otimes_{B} B^{\prime} \rightarrow \operatorname{gr}^{i}\left(\mathfrak{M}_{B^{\prime}}^{\varphi}\right) .
$$

If both $\operatorname{Tor}_{1}^{B}\left(K^{i}\left(\mathfrak{M}_{B}\right), B^{\prime}\right)$ and $\operatorname{Tor}_{1}^{B}\left(K^{i+1}\left(\mathfrak{M}_{B}\right), B^{\prime}\right)$ are zero, then we also have $\operatorname{Tor}_{1}^{B}\left(\mathfrak{M}_{B}^{\varphi} / F^{i}\left(\mathfrak{M}_{B}^{\varphi}\right), B^{\prime}\right)=0$, and so these maps are isomorphisms. As $\mathcal{G}^{i}\left(\mathfrak{M}_{B}^{\varphi}\right)$ is the cokernel of $\operatorname{gr}^{i-1}\left(\mathfrak{M}_{B}^{\varphi}\right) \rightarrow \operatorname{gr}^{i}\left(\mathfrak{M}_{B}^{\varphi}\right)$, we deduce (2) and the first part of (3). For the last part of (3), consider the following diagram for any $B$-algebra $B^{\prime}$.

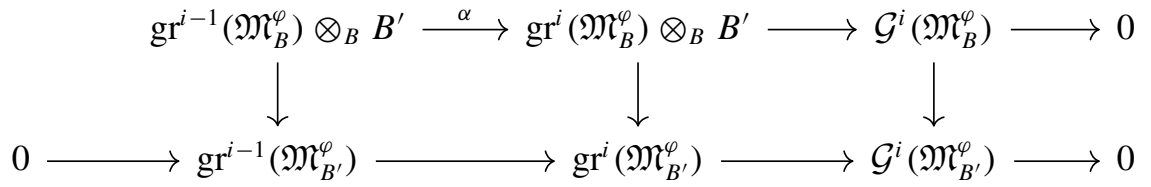

The rows are exact, and one easily checks that the squares commute. If the $K^{i}\left(\mathfrak{M}_{B}\right)$ are $B$-flat for all $i \geqslant 0$, then the vertical arrows in the diagram are isomorphisms. Hence $\alpha$ is injective and so, since the kernel of $\alpha$ identifies with $\operatorname{Tor}_{1}^{B}\left(\mathcal{G}^{i}\left(\mathfrak{M}_{B}^{\varphi}\right), B^{\prime}\right)$, we deduce each $\mathcal{G}^{i}\left(\mathfrak{M}_{B}^{\varphi}\right)$ is $B$-flat.

4.2.4. Let $A$ be a complete Noetherian ring with finite residue field $\mathbb{F}$ of characteristic $p$. Let $V_{A}$ be a finite free $A$-module equipped with a continuous $A$-linear action of $G_{K}$. To ease notation, we write $\mathcal{L}$ for the $A$-scheme $\mathcal{L}_{A \text {,crys }}^{\leqslant p}$ from Corollary 2.2.11.

LEMMA 4.2.5. For $i \geqslant 0$, there is a coherent sheaf $K^{i}$ on $\mathcal{L}$ with the following property: for any morphism Spec $B \rightarrow \mathcal{L}$ of $A$-schemes, with $B$ either finite free over $\mathbb{Z}_{p}$ or such that $\mathfrak{m}_{A}^{n} B=0$ for some $n \geqslant 1$, let $\mathfrak{M}_{B} \in \mathcal{L}_{\text {crys }}^{\leqslant p}\left(V_{B}\right)$ be the associated Breuil-Kisin module. Then the global sections of the pullback of $K^{i}$ to Spec $B$ are computed by $K^{i}\left(\mathfrak{M}_{B}\right)$. 
Proof. Since the formation of $K^{i}(-)$ is compatible with base change (being the cokernel of a map between modules compatible with base change), we obtain a compatible system of coherent sheaves on $\mathcal{L} \otimes_{A} A / \mathfrak{m}_{A}^{n}$ for $n \geqslant 1$. Grothendieck's existence theorem $[18,5.1 .6]$ (see also [28, Tag 088C]) produces the sheaf $K^{i}$ on $\mathcal{L}$ with the desired properties.

The following is the key lemma and, unlike the previous results of this section, crucially uses the assumption that $K / \mathbb{Q}_{p}$ is unramified and that we restrict to weights $\leqslant p$.

LEMma 4.2.6. Let $\mathcal{L}^{\circ} \rightarrow \mathcal{L}$ denote the closed immersion defined by the ideal consisting of sections that are either nilpotent or p-power torsion. If $p=2$, assume that

$$
K_{\infty} \cap K\left(\mu_{p^{\infty}}\right)=K
$$

(In [29, Lemma 2.1], it is shown that the compatible system $\pi^{1 / p^{\infty}}$ from 2.1 .1 can always be chosen so that $K_{\infty} \cap K\left(\mu_{p^{\infty}}\right)=K$.) Then the pullback of $K^{i}$ to $\mathcal{L}^{\circ}$ is flat.

Proof. It suffices to prove flatness at the closed points of $\mathcal{L}^{\circ}$. Thus, for a closed point $x \in \mathcal{L}$, it suffices to show $K^{i}\left(\mathfrak{M}_{B}\right)$ is $B$-flat whenever $B=\mathcal{O}_{\mathcal{L}, x} / \mathfrak{m}_{\mathcal{L}, x}^{n}$ for some $n \geqslant 1$ and any $\mathfrak{M}_{B} \in \mathcal{L}_{\text {crys }}^{\leqslant p}\left(V_{B}\right)$. By definition, $\mathcal{O}_{\mathcal{L}, x}$ is $\mathbb{Z}_{p}$-flat and reduced. It is also Nagata (since it is a localization of a finite type algebra over a complete local ring; see [28, Tag 032E]). Therefore Lemma 4.1.2, applied with $A=\mathcal{O}_{\mathcal{L}, x}$, reduces the problem to that of showing $K^{i}\left(\mathfrak{M}_{C}\right)$ is $C$-flat whenever $C$ is a finite flat $\mathbb{Z}_{p}$-algebra. This is the case by Lemma 4.2.7 (this is where the assumption that $K_{\infty} \cap K\left(\mu_{p^{\infty}}\right)=K$ when $p=2$ is used).

LEMMA 4.2.7. Let $C$ be a local finite flat $\mathbb{Z}_{p}$-algebra, and suppose $\mathfrak{M}_{C} \in$ $\mathcal{L}_{\text {crys }}^{\leqslant p}\left(V_{C}\right)$ for some continuous representation $V_{C}$ of $G_{K}$ on a finite free $C$-module. If $p=2$, assume that $K_{\infty} \cap K\left(\mu_{p^{\infty}}\right)=K$. Then $K^{i}\left(\mathfrak{M}_{C}\right)$ is $C$-flat.

Proof. It suffices to show $K^{i}\left(\mathfrak{M}_{C}\right)$ is $\mathbb{Z}_{p}$-flat and that

$$
K^{i}\left(\mathfrak{M}_{C} \otimes_{C} C / p C\right)=K^{i}\left(\mathfrak{M}_{C}\right) \otimes_{C} C / p C
$$

is $C / p C$-flat; see [28, Tag 00ML]. If $p>2$ then, since $V_{C}[1 / p]$ is crystalline, [16, Theorem 4.20] ensures the existence of an $\mathfrak{S}$-basis $\left(e_{j}\right)$ of $\mathfrak{M}_{C}$ such that $\mathfrak{M}_{C}^{\varphi}$ is generated over $\mathfrak{S}$ by $E(u)^{r_{i}} e_{i}$ for certain integers $r_{i}$. If $p=2$ and $K_{\infty} \cap K\left(\mu_{p^{\infty}}\right)=K$, then the same is true, as explained in [29, Section 4]. This implies $K^{i}\left(\mathfrak{M}_{C}\right)$ is $p$-torsion free. 
To show flatness modulo $p$, set $B=C / p C$ and let $B \rightarrow B^{\prime}$ be a surjective homomorphism. After Lemma 4.2.3, it suffices to show the natural map $F^{i}\left(\mathfrak{M}_{B}\right) \rightarrow F^{i}\left(\mathfrak{M}_{B^{\prime}}\right)$ is surjective. Proposition 3.3.1 implies $\mathfrak{M}_{B}$ is a strongly divisible. It follows from $[2,5.4 .6]$ and $[2,5.4 .2]$ that if $\mathfrak{M}$ is any strongly divisible Breuil-Kisin module and $\mathfrak{M} \rightarrow \mathfrak{N}$ is a $\varphi$-equivariant surjection into a Breuil-Kisin module that is free as a $k[[u]]$-module, then $F^{i}(\mathfrak{M}) \rightarrow F^{i}(\mathfrak{N})$ is surjective. Applying this with $\mathfrak{M}=\mathfrak{M}_{B}$ and $\mathfrak{N}=\mathfrak{M}_{B^{\prime}}$ proves the lemma.

REMARK 4.2.8. Note the second paragraph in the proof of Lemma 4.2.7 implies $K^{i}$ is flat on $\mathcal{L} \otimes_{A} A / \mathfrak{m}_{A}$. Thus it seems possible that $K^{i}$ is flat on the whole of $\mathcal{L}$ though we do not know how to prove this.

COROLlARY 4.2.9. In the situation of Lemma 4.2.6 there is, for each $i \geqslant 0$, a coherent sheaf $\mathcal{G}^{i}$ on $\mathcal{L}^{\circ}$ with the following properties. For any morphism $\operatorname{Spec} B \rightarrow \mathcal{L}^{\circ}$ of $A$-schemes, with $B$ either finite free over $\mathbb{Z}_{p}$ or such that $\mathfrak{m}_{A}^{n} B=0$ for some $n \geqslant 1$, let $\mathfrak{M}_{B} \in \mathcal{L}_{\text {crys }}^{\leqslant p}\left(V_{B}\right)$ be the associated Breuil-Kisin module. Then the global sections of the pullback of $\mathcal{G}^{i}$ to $\operatorname{Spec} B$ are computed by $\mathcal{G}^{i}\left(\mathfrak{M}_{B}\right)$. Furthermore, $\mathcal{G}^{i}$ is flat on $\mathcal{L}^{\circ}$.

Proof. Since $K^{i}$ is flat on $\mathcal{L}^{\circ}$, Lemma 4.2.3 implies that on each $\mathcal{L}^{\circ} \otimes_{A} A / \mathfrak{m}_{A}^{n}$, the formation of $\mathcal{G}^{i}(-)$ is compatible with base change. Thus we obtain a compatible system of coherent sheaves $\mathcal{G}^{i}$ on the $\mathcal{L}^{\circ} \otimes_{A} A / \mathfrak{m}_{A}^{n}$. By (3) of Lemma 4.2.3, we also know these sheaves are flat on $\mathcal{L}^{\circ} \otimes_{A} A / \mathfrak{m}_{A}^{n}$. Grothendieck's existence theorem [18, 5.1.6] produces a sheaf $\mathcal{G}^{i}$ as desired; that it is flat follows because each $\mathcal{G}^{i} \otimes_{A} A / \mathfrak{m}_{A}^{n}$ is flat.

4.2.10. Let $E$ be a finite extension of $\mathbb{Q}_{p}$ such that $A$ is an $\mathcal{O}_{E}$-algebra. Let us fix a $p$-adic Hodge type $\mathbf{v}$, that is a finite free $K \otimes_{\mathbb{Q}_{p}} E$-module $D_{\mathbf{v}}$ equipped with a grading $\operatorname{gr}^{i}\left(D_{\mathbf{v}}\right)$ by $K \otimes_{\mathbb{Q}_{p}} E$-submodules. (Below, when we speak of a $p$-adic Hodge type $\mathbf{v}$, the field $E$ will be implicit in the data of $\mathbf{v}$.) Assume this grading is concentrated in degree $[0, p]$. If $B$ is a finite local $E$-algebra, then we say a crystalline representation $V_{B}$ has $p$-adic Hodge type $\mathbf{v}$ if there are isomorphisms

$$
\operatorname{gr}^{i}\left(D_{\text {crys }}\left(V_{B}\right)\right) \stackrel{\sim}{\rightarrow} \operatorname{gr}^{i}\left(D_{\mathbf{v}}\right) \otimes_{E} B
$$

for all $i \in \mathbb{Z}$. Since we are assuming that $K$ is unramified over $\mathbb{Q}_{p}, p$-adic Hodge types can be described integrally: there exists a finite free $\mathcal{O}_{K} \otimes_{\mathbb{Z}_{p}} \mathcal{O}_{E}$-module $D_{\mathbf{v}}^{\circ}$ with a grading $\operatorname{gr}^{i}\left(D_{\mathbf{v}}^{\circ}\right)$ by $\mathcal{O}_{K} \otimes_{\mathbb{Z}_{p}} \mathcal{O}_{E}$-submodules, so that $D_{\mathbf{v}} \cong D_{\mathbf{v}}^{\circ} \otimes_{\mathcal{O}_{K}} K$ as graded modules. This is because $\mathcal{O}_{K}$ is unramified over $\mathbb{Z}_{p}$ and so $\mathcal{O}_{K} \otimes_{\mathbb{Z}_{p}} \mathcal{O}_{E}$ is a product of unramified extensions of $\mathcal{O}_{E}$. 
4.2.11. Let $B$ be a $\mathbb{Z}_{p}$-algebra. Since $\mathcal{O}_{K}$ is unramified over $\mathbb{Z}_{p}$, a finite $\mathcal{O}_{K} \otimes_{\mathbb{Z}_{p}}$ $B$-module that is flat over $B$ is flat over $\mathcal{O}_{K} \otimes_{\mathbb{Z}_{p}} B$ (see [28, Tag 00MH]). Provided $K_{\infty} \cap K\left(\mu_{p^{\infty}}\right)=K$ if $p=2$, this implies that the flat coherent sheaf $\mathcal{G}^{i}$ on $\mathcal{L}^{\circ}$

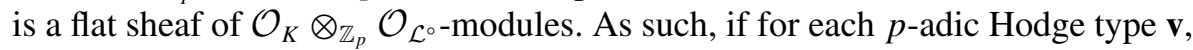
we define $\mathcal{L}^{\mathrm{v}}$ to be the set of $x \in \mathcal{L}^{\circ}$ with

$$
\mathcal{G}_{x}^{i} \cong \operatorname{gr}^{i}\left(D_{\mathbf{v}}^{\circ}\right) \otimes_{\mathcal{O}_{E}} \mathcal{O}_{\mathcal{L}^{\circ}, x}
$$

as $\mathcal{O}_{K} \otimes_{\mathbb{Z}_{p}} \mathcal{O}_{\mathcal{L}, x}$-modules for each $i \geqslant 0$, then $\mathcal{L}^{\mathbf{v}}$ is a union of connected components of $\mathcal{L}^{\circ}$.

Proposition 4.2.12. If $p=2$, assume that $K_{\infty} \cap K\left(\mu_{p^{\infty}}\right)=K$. Let $A_{\text {crys }}^{\mathrm{v}}$ denote the quotient of $A$ corresponding to the scheme-theoretic image of $\mathcal{L}^{\mathbf{v}} \rightarrow \operatorname{Spec} A$. Then, we have the following:

(1) The morphism $\mathcal{L}^{\mathbf{v}} \rightarrow \operatorname{Spec} A_{\text {crys }}^{\mathbf{v}}$ becomes an isomorphism after inverting $p$.

(2) For any finite reduced $\mathbb{Q}_{p}$-algebra $B$, a map $A \rightarrow B$ factors through $A_{\text {crys }}^{\mathbf{v}}$ if and only if $V_{B}=V_{A} \otimes_{A} B$ is crystalline with p-adic Hodge type $\mathbf{v}$.

By construction, $A_{\text {crys }}^{\mathrm{v}}$ is reduced and $\mathbb{Z}_{p}$-flat and so (2) uniquely determines this quotient.

Proof. For (1) use that, after Lemma 2.2.14, $\mathcal{L}^{\circ} \rightarrow$ Spec $A$ becomes a closed immersion after inverting $p$. For (2), argue as in Proposition 2.2.15, the point being that if $C$ is a reduced finite flat $\mathbb{Z}_{p}$-algebra, then any $\mathfrak{M}_{C} \in \mathcal{L}_{\text {crys }}^{\leqslant p}\left(V_{C}\right)$ induces a $C$-valued point of $\mathcal{L}^{\circ}$, and this point factors through $\mathcal{L}^{\mathrm{v}}$ if and only if $\mathcal{G}^{i}\left(\mathfrak{M}_{C}\right) \cong \operatorname{gr}^{i}\left(D_{\mathbf{v}}^{\circ}\right)$ for each $i \geqslant 0$.

\subsection{Cyclotomic freeness}

4.3.1. For this subsection, let $\mathbb{F}$ be a finite field of characteristic $p$ and consider $Z_{\mathbb{F}}$ and $W_{\mathbb{F}}$, both finite-dimensional $\mathbb{F}$-vector spaces equipped with a continuous $\mathbb{F}$-linear action of $G_{K}$. Further, let $\mathfrak{Z} \in \mathcal{L}_{\text {crys }}^{\leqslant p}\left(Z_{\mathbb{F}}\right)$ and $\mathfrak{W} \in \mathcal{L}_{\text {crys }}^{\leqslant p}\left(W_{\mathbb{F}}\right)$. It will be useful to consider the following hypothesis.

Hypothesis 4.3.2. (1) Every continuous cocycle $G_{K} \rightarrow \operatorname{Hom}(\mathfrak{Z}, \mathfrak{W}) \bigotimes_{k[[u]]}$ $u^{p / p-1} \mathcal{O}_{C^{b}}$ given by $\sigma \mapsto F_{\sigma}$ with (i) $(\varphi-1)\left(F_{\sigma}\right)=0$ for all $\sigma \in G_{K}$, and (ii) $F_{\sigma}=0$ for all $\sigma \in G_{K_{\infty}}$, is zero.

(2) If $V_{\mathbb{F}}$ is a continuous representation of $G_{K}$ on a finite-dimensional $\mathbb{F}$-vector space and $\mathfrak{M} \in \mathcal{L}_{\text {crys }}^{\leqslant p}\left(V_{\mathbb{F}}\right)$, then (1) is satisfied when $\mathfrak{Z}=\mathfrak{W}=\mathfrak{M}$. 
Lemma 4.3.3. Suppose (1) of Hypothesis 4.3.2 is satisfied. Then, we have the following:

(1) Under the identification $\operatorname{Hom}(\mathfrak{Z}, \mathfrak{W}) \otimes_{k[[u]]} C^{b}=\operatorname{Hom}\left(Z_{\mathbb{F}}, W_{\mathbb{F}}\right) \otimes_{\mathbb{F}_{p}} C^{b}$, there are inclusions $\operatorname{Hom}(\mathfrak{Z}, \mathfrak{W})^{\varphi=1} \subset \operatorname{Hom}\left(Z_{\mathbb{F}}, W_{\mathbb{F}}\right)^{G_{K}}$.

(2) Let $0 \rightarrow W_{\mathbb{F}} \rightarrow Y \rightarrow Z_{\mathbb{F}} \rightarrow 0$ be an exact sequence of $G_{K_{\infty}}$-representations and let $\mathfrak{Y} \in \mathcal{L}_{\mathrm{SD}}^{\leqslant p}(Y)$ be such that Lemma 3.2.5 induces an exact sequence $0 \rightarrow \mathfrak{W} \rightarrow \mathfrak{Y} \rightarrow \mathfrak{Z} \rightarrow 0$. Then there exists at most one way of extending the $G_{K_{\infty}}$-action on $Y$ to a $G_{K}$-action so that $\mathfrak{Y} \in \mathcal{L}_{\text {crys }}^{\leqslant p}(Y)$ and $0 \rightarrow W_{\mathbb{F}} \rightarrow$ $Y \rightarrow Z_{\mathbb{F}} \rightarrow 0$ is $G_{K}$-equivariant.

Proof. For (1), note that in general $\operatorname{Hom}(\mathfrak{Z}, \mathfrak{W})^{\varphi=1} \subset \operatorname{Hom}\left(Z_{\mathbb{F}}, W_{\mathbb{F}}\right)^{G_{K_{\infty}}}$. As a consequence, if $f \in \operatorname{Hom}(\mathfrak{Z}, \mathfrak{W})^{\varphi=1}$, then the 1-cocycle $\sigma \mapsto(\sigma-1)(f)$ satisfies the conditions of Hypothesis 4.3.2. Thus $(\sigma-1)(f)=0$ and so $f$ is $G_{K}$-equivariant.

For (2), recall from 3.3.6 that, after choosing an $\mathfrak{S}_{\mathbb{F}}$ splitting of $0 \rightarrow \mathfrak{W} \rightarrow$ $\mathfrak{Y} \rightarrow \mathfrak{Z} \rightarrow 0$ so that $\varphi_{\mathfrak{Y}}=\left(\varphi_{\mathfrak{W}}+f \circ \varphi_{\mathfrak{Z}}, \varphi_{\mathfrak{Z}}\right)$, the possible ways of extending the $G_{K_{\infty}}$-action on $Y$ to a $G_{K}$-action as required by the lemma are in bijection with the set of 1-cocycles $\sigma \mapsto f_{\sigma}$ taking values in $\operatorname{Hom}(\mathfrak{Z}, \mathfrak{W}) \otimes_{k[u]]} u^{p / p-1} \mathcal{O}_{C^{b}}$ and satisfying $(\varphi-1)\left(f_{\sigma}\right)=(\sigma-1)(f)$ and $f_{\sigma}=0$ for $\sigma \in G_{K_{\infty}}$. As the difference of two such cocycles is a cocycle as in Hypothesis 4.3.2, we obtain 2.

LEMMA 4.3.4. Let $0 \rightarrow Z_{\mathbb{F}, 1} \rightarrow Z_{\mathbb{F}} \rightarrow Z_{\mathbb{F}, 2} \rightarrow 0$ be a $G_{K}$-equivariant exact sequence, and suppose $0 \rightarrow \mathfrak{Z}_{1} \rightarrow \mathfrak{Z} \rightarrow \mathfrak{Z}_{2} \rightarrow 0$ is the corresponding $\varphi$ equivariant exact sequence from Lemma 3.2.5. If (1) of Hypothesis 4.3.2 is satisfied when $\mathfrak{Z}$ is replaced by $\mathfrak{Z}_{1}$ and $\mathfrak{Z}_{2}$, then (1) of Hypothesis 4.3 .2 is satisfied itself.

Proof. Applying $\operatorname{Hom}(-, \mathfrak{W})$ to $0 \rightarrow \mathfrak{Z}_{1} \rightarrow \mathfrak{Z} \rightarrow \mathfrak{Z}_{2} \rightarrow 0$ yields a $\varphi$-equivariant exact sequence

$$
0 \rightarrow \operatorname{Hom}\left(\mathfrak{Z}_{1}, \mathfrak{W}\right) \rightarrow \operatorname{Hom}(\mathfrak{Z}, \mathfrak{W}) \rightarrow \operatorname{Hom}\left(\mathfrak{Z}_{2}, \mathfrak{W}\right) \rightarrow 0,
$$

which is $G_{K}$-equivariant after applying $\otimes_{k[u] l} C^{b}$. Thus, if $\sigma \mapsto F_{\sigma}$ is a 1-cocycle as in Hypothesis 4.3.2, then so is its image in $\operatorname{Hom}\left(\mathfrak{Z}_{2}, \mathfrak{W}\right) \otimes_{k[[u]]} u^{p / p-1} \mathcal{O}_{C^{b}}$. We conclude that if (1) of Hypothesis 4.3.2 is satisfied with $\mathfrak{Z}$ replaced by $\mathfrak{Z}_{2}$, then this image must be zero. Sequence 4.3.5 remains exact after applying $\otimes_{k[[u]]} u^{p / p-1} \mathcal{O}_{C^{b}}$ since each of its terms is $k[[u]]$-free. Therefore $F_{\sigma} \in \operatorname{Hom}\left(\mathfrak{Z}_{1}\right.$, $\mathfrak{W}) \otimes_{k[u]]} u^{p / p-1} \mathcal{O}_{C^{b}}$ for each $\sigma$. If (1) of Hypothesis 4.3.2 is satisfied with $\mathfrak{Z}$ replaced by $\mathfrak{Z}_{1}$, then we must have $F_{\sigma}=0$. Hence (1) of Hypothesis 4.3.2 itself is satisfied. 
Proposition 4.3.6. Hypothesis 4.3.2(1) is satisfied when either of the following two conditions holds:

(1) $\mathfrak{Z} \in \mathcal{L}_{\text {crys }}^{\leqslant p-1}\left(Z_{\mathbb{F}}\right)$.

(2) Every Jordan-Holder factor of $Z_{\mathbb{F}}$ is absolutely irreducible and if $Z$ is a Jordan-Holder factor of $Z_{\mathbb{F}}$ so that $Z \otimes_{\mathbb{F}} \overline{\mathbb{F}}(1)$ is unramified, then $Z \otimes_{\mathbb{F}} \overline{\mathbb{F}}(1)$ is not a Jordan-Holder factor of $W_{\mathbb{F}} \otimes_{\mathbb{F}} \overline{\mathbb{F}}$. (Here $\overline{\mathbb{F}}(1)$ denotes the one-dimensional representation of $G_{K}$ over $\overline{\mathbb{F}}$ on which $G_{K}$-acts by the cyclotomic character. Likewise for $\overline{\mathbb{F}}(-1)$, but for the inverse of the cyclotomic character.)

Proof. By inducting on the length of $Z_{\mathbb{F}}$, and using Lemma 4.3.4, we can reduce to the case that $Z_{\mathbb{F}}$ is irreducible. Let $\sigma \mapsto F_{\sigma}$ be as in Hypothesis 4.3.2 and suppose $\sigma \in G_{K}$ is such that $F_{\sigma} \neq 0$. Let $\mathfrak{J}$ be the kernel of the restriction of $F_{\sigma}$ to $\mathfrak{Z}$. Since $F_{\sigma}$ is $\varphi$-equivariant, $\mathfrak{J}$ is a $\varphi$-stable $\mathfrak{S}_{\mathbb{F}}$-submodule of $\mathfrak{Z}$. Since the image of $F_{\sigma}$ is $u$-torsion-free, $\mathfrak{J} \otimes_{k[u]]} C^{b}=\mathfrak{Z} \otimes_{k[[u]]} C^{b}$ only if $\mathfrak{J}=\mathfrak{Z}$, and this does not happen since $F_{\sigma} \neq 0$. Since $Z_{\mathbb{F}}$ is irreducible as a $G_{K}$-representation, it is irreducible as a $G_{K_{\infty}}$-representation; see 3.2.1. Therefore $\mathfrak{J}=0$ and $F_{\sigma}$ is injective; otherwise, the $G_{K_{\infty}}$-representation $\left(\mathfrak{J} \otimes_{k[[u]]} C^{b}\right)^{\varphi=1} \subset Z_{\mathbb{F}}$ contradicts the $G_{K_{\infty}}$-irreducibility of $Z_{\mathbb{F}}$.

For each $z \in \mathfrak{Z} \backslash u \mathfrak{Z}$ and each $n \geqslant 1$, there exist $\delta_{n} \in \mathbb{Z}$ and $z_{n} \in \mathfrak{Z} \backslash u \mathfrak{Z}$ such that

$$
\varphi^{n}(z)=u^{p^{n}+\cdots+p-\delta_{n}} z_{n} .
$$

Using that $u^{p} \mathfrak{Z} \subset \mathfrak{Z}^{\varphi}$, we deduce that $\delta_{n} \geqslant 0$ (the point being that $\varphi(z) \notin u^{p+1} \mathfrak{Z}$ if it was then $\varphi(z) / u \in \mathfrak{Z}^{\varphi}$, which implies $z \in u^{1 / p} \mathfrak{Z} \otimes_{k[[u]]} \mathcal{O}_{C^{b}}$, a contradiction). In particular, there is a $\delta^{\prime} \geqslant 0$ such that $\varphi\left(z_{n}\right)=u^{p-\delta^{\prime}} z_{n+1}$ and so, since

$$
\varphi^{n+1}(z)=u^{p^{n+1}+\cdots+p^{2}-p \delta_{n}} \varphi\left(z_{n}\right)=u^{p^{n+1}+\cdots+p^{2}+p-p \delta_{n}-\delta^{\prime}} z_{n+1},
$$

we see that $\delta_{n+1}=p \delta_{n}+\delta^{\prime} \geqslant p \delta_{n}$. In particular, if $\delta_{N}>0$ for some $N$, then $\delta_{n} \rightarrow \infty$ as $n \rightarrow \infty$. As $F_{\sigma}$ is injective and $\mathfrak{Z}$ is finitely generated, there exists $\gamma>0$ such that $F_{\sigma}(z) \notin \mathfrak{W} \otimes_{k[[u]]} u^{\gamma+p /(p-1)} \mathcal{O}_{C^{b}}$ for any $z \in \mathfrak{Z} \backslash u \mathfrak{Z}$. This implies

$$
F_{\sigma}\left(\varphi^{n}(z)\right)=u^{p^{n}+\cdots+p-\delta_{n}} F_{\sigma}\left(z_{n}\right) \notin \mathfrak{W} \otimes_{k[[u]]} u^{p^{n}+\cdots+p-\delta_{n}+\gamma+p /(p-1)} \mathcal{O}_{C^{b}}
$$

for any $z \in \mathfrak{Z} \backslash u \mathfrak{Z}$ and $n \geqslant 0$. As $F_{\sigma}$ is $\varphi$-equivariant and $F_{\sigma} \in \operatorname{Hom}(\mathfrak{Z}, \mathfrak{W}) \bigotimes_{k[[u]]}$ $u^{p /(p-1)} \mathcal{O}_{C^{b}}$, we also deduce

$$
F_{\sigma}\left(\varphi^{n}(z)\right)=\varphi^{n}\left(F_{\sigma}(z)\right) \in \mathfrak{W} \otimes_{k[[u]]} u^{p^{n+1} /(p-1)} \mathcal{O}_{C^{b}} .
$$

Note that $p^{n}+\cdots+p-\delta_{n}+\gamma-p /(p-1)=p^{n+1} /(p-1)-\delta_{n}+\gamma$. If $\delta_{N}>0$ for some $N$ then, by choosing $n$ large enough that $-\delta_{n}+\gamma<0$, we obtain 
a contradiction. We conclude that $F_{\sigma}=0$ unless, for all $z \in \mathfrak{Z} \backslash u \mathfrak{Z}, \varphi(z)=u^{p} z^{\prime}$ for some $z^{\prime} \in \mathfrak{Z} \backslash u \mathfrak{Z}$. Equivalently, $F_{\sigma}=0$ unless $\mathfrak{Z}^{\varphi}=u^{p} \mathfrak{Z}$.

This completes the proof when $\mathfrak{Z} \in \mathcal{L}_{\text {crys }}^{\leqslant p-1}\left(Z_{\mathbb{F}}\right)$ since then $u^{p-1} \mathfrak{Z} \subset \mathfrak{Z}^{\varphi}$. Therefore assume we are as in (2). If $\mathfrak{Z}^{\varphi}=u^{p} \mathfrak{Z}$, then $\varphi$ induces a semilinear automorphism of $u^{-p / p-1} \mathfrak{Z}$, and hence a $\bar{k}$-semilinear automorphism of $\widetilde{\mathfrak{Z}}:=$ $\left(u^{p / p-1} \mathfrak{Z}\right) \otimes_{k[u]]} \bar{k}$. Via the $\varphi$-equivariant section of $\mathcal{O}_{C^{b}} \rightarrow \bar{k}$ given by Teichmuller representatives, we $\varphi$-equivariantly view $\widetilde{\mathfrak{Z}}$ as a subset of $\mathfrak{Z} \otimes_{k[[u]]} C^{b}$. Since $\bar{k}$ is algebraically closed, $\widetilde{\mathfrak{Z}}$ is generated by $\varphi$-invariant elements, and so $\widetilde{\mathfrak{Z}}^{\varphi=1}=Z_{\mathbb{F}}$. It is a straightforward exercise to show that, as a $G_{K_{\infty}}$-representation, $\widetilde{\mathfrak{Z}}^{\varphi=1}$ is a twist of an unramified representation by the inverse of the cyclotomic character. Thus $\widetilde{\mathfrak{Z}}^{\varphi=1}$ has the same description as a $G_{K}$-representation; see 3.2.1. As $Z_{\mathbb{F}}$ is absolutely irreducible, it follows that $Z_{\mathbb{F}}$ is one-dimensional.

We have shown that if a nonzero cocycle $\sigma \mapsto F_{\sigma}$ exists as in Hypothesis 4.3.2, then $Z_{\mathbb{F}} \otimes_{\mathbb{F}} \overline{\mathbb{F}}(1)$ is an unramified character. Since $\left.F_{\sigma}\right|_{G_{K_{\infty}}}=0$, this cocycle represents a class in $H^{1}\left(G_{K}, \operatorname{Hom}\left(Z_{\mathbb{F}} \otimes_{\mathbb{F}} \overline{\mathbb{F}}, W_{\mathbb{F}} \otimes_{\mathbb{F}} \overline{\mathbb{F}}\right)\right)$, which is killed by restriction to $G_{K_{\infty}}$. If $Z_{\mathbb{F}} \otimes_{\mathbb{F}} \overline{\mathbb{F}}(1)$ is not a Jordan-Holder factor of $W_{\mathbb{F}} \otimes_{\mathbb{F}} \overline{\mathbb{F}}$, then $[3,2.3 .5]$ implies this restriction map is injective, so $F_{\sigma}=(\sigma-1)(F)$ for some $F \in \operatorname{Hom}\left(Z_{\mathbb{F}} \otimes_{\mathbb{F}} \overline{\mathbb{F}}, W_{\mathbb{F}} \otimes_{\mathbb{F}} \overline{\mathbb{F}}\right)$, which is fixed by $G_{K_{\infty}}$. Applying [3, 2.3.5] again then implies $F$ is fixed by $G_{K}$, so $F_{\sigma}=0$. We conclude (1) of Hypothesis 4.3 .2 is satisfied.

This motivates the following definition.

DEFINITION 4.3.7. (1) We say $V_{\mathbb{F}}$ is cyclotomic-free if every Jordan-Holder factor of $V_{\mathbb{F}}$ is absolutely irreducible, and if $Z$ is a Jordan-Holder factor of $V_{\mathbb{F}}$ such that $Z \otimes_{\mathbb{F}} \overline{\mathbb{F}}(1)$ is unramified then $Z \otimes_{\overline{\mathbb{F}}} \overline{\mathbb{F}}(1)$ is not a Jordan-Holder factor of $V_{\mathbb{F}} \otimes_{\mathbb{F}} \overline{\mathbb{F}}$.

(2) We say $V_{\mathbb{F}}$ is strongly cyclotomic-free if $\left.V_{\mathbb{F}}\right|_{G_{L}}$ is cyclotomic-free for all finite unramified extensions $L / K$. Equivalently, $V_{\mathbb{F}}$ is strongly cyclotomicfree if each Jordan-Holder factor is absolutely irreducible, and if an unramified twist of $\mathbb{F}(-1)$ is a Jordan-Holder factor of $V_{\mathbb{F}}$ then no JordanHolder factor of $V_{\mathbb{F}}$ is unramified.

Most of our results will only require us to assume cyclotomic freeness. However, to prove potential diagonalizability, it will be necessary to replace a representation $V_{\mathbb{F}}$ by the restriction $\left.V_{\mathbb{F}}\right|_{G_{L}}$ for some sufficiently large unramified extension $L / K$ so that $\left.V_{\mathbb{F}}\right|_{G_{L}}$ has every Jordan-Holder factor one-dimensional. To apply our results, we will need $\left.V_{\mathbb{F}}\right|_{G_{L}}$ to be cyclotomic-free. The following example indicates why we therefore require $V_{\mathbb{F}}$ to satisfy a stronger property than cyclotomic freeness. 
EXAMPLE 4.3.8. Assume $p=2$ and let $\psi$ be a nontrivial character of $G_{K}$, which becomes trivial when restricted to $G_{L}$ for $L / K$ a finite unramified extension. Let $V_{\mathbb{F}}$ be an irreducible representation of $G_{K}$ of dimension $[L: K]$. Assuming $\mathbb{F}$ to be sufficiently large, $L / K$ is then the smallest unramified extension such that $\left.V_{\mathbb{F}}\right|_{G_{L}}$ has every Jordan-Holder factor one-dimensional. However, if $V_{\mathbb{F}}^{\prime}=V_{\mathbb{F}} \oplus \mathbb{F}(\psi)$, then $\left.V_{\mathbb{F}}^{\prime}\right|_{G_{L}}$ is not cyclotomic-free since $\left.\mathbb{F}(\psi)\right|_{G_{L}}$ is trivial and, because $p=2$, the cyclotomic character is also trivial.

COROLlary 4.3.9. If $V_{\mathbb{F}}$ is cyclotomic-free, then (2) of Hypothesis 4.3.2 holds for all $\mathfrak{M} \in \mathcal{L}_{\text {crys }}^{\leqslant p}\left(V_{\mathbb{F}}\right)$.

Proof. This follows from Proposition 4.3.6 applied with $\mathfrak{Z}=\mathfrak{W}=\mathfrak{M}$.

\subsection{Local analysis of $\mathcal{L}_{\text {crys }}^{\leqslant p}$}

4.4.1. With notation as in 4.3.1, a deformation of $V_{\mathbb{F}}$ to a complete local $W(\mathbb{F})$-algebra $A$, with residue field $\mathbb{F}$, is a finite free $A$-module $V_{A}$ equipped with a continuous $A$-linear action of $G_{K}$ together with a $G_{K}$-equivariant isomorphism $V_{A} \otimes_{A} \mathbb{F} \cong V_{\mathbb{F}}$.

Fix an $\mathbb{F}$-basis $\xi_{\mathbb{F}}$ of $V_{\mathbb{F}}$. Then a framed deformation of $V_{\mathbb{F}}$ is a deformation $V_{A}$ together with an $A$-basis $\xi_{A}$, which gets identified with $\xi_{\mathbb{F}}$ after applying $\otimes_{A} \mathbb{F}$. The functor

$$
\left.D_{V_{\mathbb{F}}}^{\square}(A)=\text { isomorphism classes of framed deformations of } V_{\mathbb{F}}\right\}
$$

is representable by a complete local Noetherian $W(\mathbb{F})$-algebra $R=R_{V_{\mathbb{F}}}^{\square}$. Let $V_{R}$ denote the universal framed deformation. Applying Corollary 2.2.11 to $R$ and $V_{R}$ gives a projective $R$-scheme $\mathcal{L}:=\mathcal{L}_{R \text {,crys }}^{\leqslant p}$.

LEMMA 4.4.2. Let $\mathbb{F}^{\prime}$ be a finite extension of $\mathbb{F}$ and $x$ an $\mathbb{F}^{\prime}$-valued point of $\mathcal{L}$. Suppose the corresponding $\mathfrak{M}_{x} \in \mathcal{L}_{\text {crys }}^{\leqslant p}\left(V_{\mathbb{F}^{\prime}}\right)$ satisfies (2) of Hypothesis 4.3.2. Then (It would be better to write $\sum_{n+m=i} \operatorname{dim}_{\mathbb{F}} \mathcal{G}^{n}\left(\mathfrak{M}_{x}\right)-\operatorname{dim}_{\mathbb{F}} \mathcal{G}^{m}\left(\mathfrak{M}_{x}\right)$ as $\mathcal{G}^{i}\left(\operatorname{Hom}\left(\mathfrak{M}_{x}, \mathfrak{M}_{x}\right)\right)$, but we have only defined $\mathcal{G}^{i}(-)$ for finite projective $\mathfrak{S}_{\mathbb{F}}$-modules equipped with maps $\varphi^{*} \mathfrak{M} \rightarrow \mathfrak{M}$. The image of the Frobenius on $\operatorname{Hom}\left(\mathfrak{M}_{x}, \mathfrak{M}_{x}\right)$ will not, in general, be contained in $\operatorname{Hom}\left(\mathfrak{M}_{x}, \mathfrak{M}_{x}\right)$.)

$$
\operatorname{dim}_{\mathbb{F}^{\prime}} \mathcal{O}_{\mathcal{L}, x}\left(\mathbb{F}^{\prime}[\epsilon]\right) \leqslant d^{2}+\sum_{i>0} \sum_{n-m=i} \operatorname{dim}_{\mathbb{F}^{\prime}} \mathcal{G}^{n}\left(\mathfrak{M}_{x}\right)-\operatorname{dim}_{\mathbb{F}^{\prime}} \mathcal{G}^{m}\left(\mathfrak{M}_{x}\right)
$$

Here $\mathbb{F}^{\prime}[\epsilon]$ is the ring of dual numbers over $\mathbb{F}^{\prime}$. 
Proof. Replacing $V_{\mathbb{F}}$ by $V_{\mathbb{F}} \otimes_{\mathbb{F}} \mathbb{F}^{\prime}$, we may suppose $\mathbb{F}=\mathbb{F}^{\prime}$. An element of $\mathcal{O}_{\mathcal{L}, x}(\mathbb{F}[\epsilon])$ gives rise to a map $R \rightarrow \mathbb{F}[\epsilon]$, and so a framed deformation $V_{\mathbb{F}[\epsilon]}$ of $V_{\mathbb{F}}$ to $\mathbb{F}[\epsilon]$, and an element $\mathfrak{M}_{\mathbb{F}[\epsilon]} \in \mathcal{L}_{\text {crys }}^{\leqslant p}\left(V_{\mathbb{F}[\epsilon]}\right)$ satisfying $\mathfrak{M}_{\mathbb{F}[\epsilon]} \otimes_{\mathbb{F}[\epsilon]} \mathbb{F}=\mathfrak{M}_{x}$. When viewed as an $\mathfrak{S}_{\mathbb{F}}$-module, $\mathfrak{M}_{\mathbb{F}[\epsilon]}$ fits into an exact sequence

$$
0 \rightarrow \mathfrak{M}_{x} \stackrel{\epsilon}{\rightarrow} \mathfrak{M}_{\mathbb{F}[\epsilon]} \rightarrow \mathfrak{M}_{x} \rightarrow 0 .
$$

As explained in Proposition 3.3.5, an $\mathfrak{S}_{\mathbb{F}}$-splitting of this sequence can be chosen so that $\varphi_{\mathfrak{M}_{\mathbb{F}[\epsilon]}}=\left(\varphi_{\mathfrak{M}_{x}}+f \circ \varphi_{\mathfrak{M}_{x}}, \varphi_{\mathfrak{M}_{x}}\right)$ for some $f \in \operatorname{Hom}\left(\mathfrak{M}_{x}, \mathfrak{M}_{x}\right)$. Since choosing a different splitting replaces $f$ by $f+(\varphi-1)(g)$ for some $g \in \operatorname{Hom}\left(\mathfrak{M}_{x}\right.$, $\mathfrak{M}_{x}$ ), we obtain a well-defined map

$$
\mathcal{O}_{\mathcal{L}, x}(\mathbb{F}[\epsilon]) \rightarrow \operatorname{Hom}\left(\mathfrak{M}_{x}, \mathfrak{M}_{x}\right) / F^{0} \operatorname{Hom}\left(\mathfrak{M}_{x}, \mathfrak{M}_{x}\right),
$$

where $F^{0} \operatorname{Hom}\left(\mathfrak{M}_{x}, \mathfrak{M}_{x}\right)$ consists of those $g \in \operatorname{Hom}\left(\mathfrak{M}_{x}, \mathfrak{M}_{x}\right)$ for which $\varphi(g) \in$ $\operatorname{Hom}\left(\mathfrak{M}_{x}, \mathfrak{M}_{x}\right)$. We remark that the target of (4.4.3) can be identified with $\operatorname{Ext}_{\mathrm{SD}}^{1}\left(\mathfrak{M}_{x}, \mathfrak{M}_{x}\right)$, the first Yoneda extension group in the exact category of strongly divisible Breuil-Kisin modules; see [3, Section 4.1]. It is easy to check that this map is $\mathbb{F}$-linear.

If $\mathcal{W}$ is the multiset of integers containing $i$ with multiplicity equal to the $\mathbb{F}$-dimension of $\mathcal{G}^{i}\left(\mathfrak{M}_{x}\right)$, then $[3,4.2 .5]$ implies the right-hand side of (4.4.3) has $\mathbb{F}$-dimension

$$
\operatorname{dim}_{\mathbb{F}} \operatorname{Hom}\left(\mathfrak{M}_{x}, \mathfrak{M}_{x}\right)^{\varphi=1}+\operatorname{Card}\{i-j>0 \mid i, j \in \mathcal{W}\} .
$$

Clearly, the value of the double sum in the statement of the lemma equals the cardinality of $\{i-j>0 \mid i, j \in \mathcal{W}\}$, and so it remains to bound the dimension of the kernel of (4.4.3). To do this, we first claim this kernel is contained in the kernel of the composite

$$
\mathcal{O}_{\mathcal{L}, x}(\mathbb{F}[\epsilon]) \rightarrow R(\mathbb{F}[\epsilon]) \rightarrow \operatorname{Ext}^{1}\left(V_{\mathbb{F}}, V_{\mathbb{F}}\right) .
$$

Here the last maps sends $R \rightarrow \mathbb{F}[\epsilon]$ onto the exact sequence $0 \rightarrow V_{\mathbb{F}} \stackrel{\epsilon}{\rightarrow} V_{R} \otimes_{R}$ $\mathbb{F}[\epsilon] \rightarrow V_{\mathbb{F}} \rightarrow 0$. If $\mathfrak{M}_{\mathbb{F}[\epsilon]}$ corresponds to an element in the kernel of (4.4.3), then the surjection $\mathfrak{M}_{\mathbb{F}[\epsilon]} \rightarrow \mathfrak{M}_{x}$ admits a $\varphi$-equivariant $\mathfrak{S}_{\mathbb{F}}$-linear splitting $s$. Since $\mathfrak{M}_{\mathbb{F}[\epsilon]} \rightarrow \mathfrak{M}$ becomes $G_{K}$-equivariant after applying $\otimes_{k[[u]} C^{\mathrm{b}}$, it follows that $(\sigma-1)(s):=\sigma \circ s \circ \sigma^{-1}-s$ is an element of $\operatorname{Hom}\left(\mathfrak{M}_{x}, \mathfrak{M}_{x}\right) \bigotimes_{k[u]]} u^{p /(p-1)} \mathcal{O}_{C^{b}}$ for each $\sigma \in G_{K}$. Using Hypothesis 4.3.2, we deduce that $s$ is $G_{K}$-equivariant, and so $s$ induces a $G_{K}$-equivariant splitting of $V_{\mathbb{F}[\epsilon]} \rightarrow V_{\mathbb{F}}$. This proves the claim.

To finish the proof, it therefore suffices to show that the kernel of $\mathcal{O}_{\mathcal{L}, x}(\mathbb{F}[\epsilon]) \rightarrow$ $R(\mathbb{F}[\epsilon]) \rightarrow \operatorname{Ext}^{1}\left(V_{\mathbb{F}}, V_{\mathbb{F}}\right)$ has dimension equal to

$$
\leqslant d^{2}-\operatorname{Hom}\left(\mathfrak{M}_{x}, \mathfrak{M}_{x}\right)^{\varphi=1} .
$$


We claim that the kernel of the first map in this composite is a torsor for

$$
\operatorname{Hom}\left(V_{\mathbb{F}}, V_{\mathbb{F}}\right)^{G_{K}} / \operatorname{Hom}\left(\mathfrak{M}_{x}, \mathfrak{M}_{x}\right)^{\varphi=1}
$$

(note that this makes sense since by Lemma 4.3.3, we do have Hom $\left(\mathfrak{M}_{x}, \mathfrak{M}_{x}\right)^{\varphi=1}$ $\left.\subset \operatorname{Hom}\left(V_{\mathbb{F}}, V_{\mathbb{F}}\right)^{G_{K}}\right)$. Since the kernel of the second map in this composite is clearly a torsor for $\operatorname{Hom}\left(V_{\mathbb{F}}, V_{\mathbb{F}}\right) / \operatorname{Hom}\left(V_{\mathbb{F}}, V_{\mathbb{F}}\right)^{G_{K}}$, proving this claim will complete the argument.

To do this, note that any $h \in \operatorname{Hom}\left(V_{\mathbb{F}}, V_{\mathbb{F}}\right)^{G_{K}}$ produces an automorphism $a+b \epsilon \mapsto a+h(b) \epsilon$ of $V_{\mathbb{F}} \otimes_{\mathbb{F}} \mathbb{F}[\epsilon]$ which, when viewed as an automorphism of $V_{\mathbb{F}} \otimes_{\mathbb{F}} \mathbb{F}[\epsilon] \otimes_{\mathbb{F}_{p}} C^{b}$, acts on the set $X \subset \mathcal{L}_{\text {crys }}^{\leqslant p}\left(V_{\mathbb{F}} \otimes_{\mathbb{F}} \mathbb{F}[\epsilon]\right)$ containing those elements corresponding to elements in the kernel of $\mathcal{O}_{\mathcal{L}, x}(\mathbb{F}[\epsilon]) \rightarrow R(\mathbb{F}[\epsilon])$. This action is also transitive. To see this, note that any two elements of $X$ are abstractly isomorphic as Breuil-Kisin modules by a $\varphi$-equivariant map inducing the identity modulo $\epsilon$. By Lemma 4.3.3, this isomorphism induces an automorphism of $V_{\mathbb{F}} \otimes_{\mathbb{F}} \mathbb{F}[\epsilon]$ which, being the identity modulo $\epsilon$, is of the form $a+b \epsilon \mapsto a+h(b) \epsilon$ for some $h \in \operatorname{Hom}\left(V_{\mathbb{F}}, V_{\mathbb{E}}\right)^{G_{K}}$. Finally we note that $\operatorname{Hom}\left(\mathfrak{M}_{x}, \mathfrak{M}_{x}\right)^{\varphi=1}$ is the stabilizer of any point of $X$ under this action.

Proposition 4.4.4. If $p=2$, assume that $K_{\infty} \cap K\left(\mu_{p^{\infty}}\right)=K$. Let $x \in \mathcal{L}$ be an $\mathbb{F}^{\prime}$-valued closed point of $\mathcal{L}$ with $\mathcal{O}_{\mathcal{L}, x}[1 / p] \neq 0$, and assume that the corresponding element of $\mathcal{L}_{\text {crys }}^{\leqslant p}\left(V_{\mathbb{F}^{\prime}}\right)$ satisfies (2) of Hypothesis 4.3.2. Then $\mathcal{O}_{\mathcal{L}, x}$ is $\mathbb{Z}_{p}$-flat and $\mathcal{O}_{\mathcal{L}, x} / p$ is regular. The completion of $\mathcal{O}_{\mathcal{L}, x}$ is a power series over $W\left(\mathbb{F}^{\prime}\right)$.

Proof. Let $\mathfrak{p} \in \mathcal{O}_{\mathcal{L}, x}[1 / p]$ be a maximal ideal and let $\mathfrak{q}$ be its preimage in $\mathcal{O}_{\mathcal{L}, x}$. Set $B$ equal to the residue field of $\left(\mathcal{O}_{\mathcal{L}, x}\right)_{\mathfrak{q}}$ and $C=\mathcal{O}_{\mathcal{L}, x} / \mathfrak{q} \subset B$. By Lemma 4.1.1, we know $B$ is a finite extension of $\mathbb{Q}_{p}$ and $C$ is finite flat over $\mathbb{Z}_{p}$.

Let $y \in \mathcal{L}$ denote the image of $\operatorname{Spec} B \rightarrow \mathcal{L}$. Since $\mathcal{L}[1 / p]$ is Jacobson and $B$ is finite over $\mathbb{Q}_{p}, y$ is closed in $\mathcal{L}[1 / p]$; see [28, Tag 01TB]. The map Spec $C \rightarrow \mathcal{L}$ corresponds to $\mathfrak{M}_{C} \in \mathcal{L}_{\text {crys }}^{\leqslant p}\left(V_{C}\right)$ with $\mathfrak{M}_{C} \otimes_{C} \mathbb{F}^{\prime}$ the Breuil-Kisin module corresponding to $x$. Lemmas 4.2.7 and 4.2.3 imply that $\mathcal{G}^{i}\left(\mathfrak{M}_{C} \otimes_{C} \mathbb{F}^{\prime}\right) \cong$ $\mathcal{G}^{i}\left(\mathfrak{M}_{C}\right) \otimes_{C} \mathbb{F}^{\prime}$. If $V_{B}=V_{C} \otimes_{C} B$ is the representation of $G_{K}$ induced by $y$, then $V_{B}$ is crystalline and we also have $\mathcal{G}^{i}\left(\mathfrak{M}_{C}\right) \otimes_{C} B \cong \operatorname{gr}^{i}\left(D_{\mathrm{dR}}\left(V_{B}\right)\right)$; see 4.2.2. If $\mathfrak{M}_{x} \in \mathcal{L}_{\text {crys }}^{\leqslant p}\left(V_{\mathbb{F}}\right)$ corresponds to $x$, then we deduce

$$
\operatorname{dim}_{\mathbb{F}^{\prime}} \mathcal{G}^{i}\left(\mathfrak{M}_{C} \otimes_{C} \mathbb{F}^{\prime}\right)=\operatorname{dim}_{B} \operatorname{gr}^{i}\left(D_{\mathrm{dR}}\left(V_{B}\right)\right)
$$

From Proposition 2.2.15, we know $\mathcal{L}[1 / p]=\operatorname{Spec} R_{\text {crys }}^{\leqslant p}[1 / p]$. By [21, 2.6.2] and $[21,3.3 .8]$, the connected component of $R_{\text {crys }}^{\leqslant p}[1 / p]$ containing $y$ is 
equidimensional of dimension

$$
\begin{aligned}
d^{2} & +\sum_{i>0} \operatorname{dim}_{\mathbb{F}} \operatorname{gr}^{i}\left(D_{\mathrm{dR}}\left(\operatorname{Hom}\left(V_{B}, V_{B}\right)\right)\right. \\
& =d^{2}+\sum_{i>0} \sum_{n-m=i} \operatorname{dim}_{\mathbb{F}} \operatorname{gr}^{n}\left(D_{\mathrm{dR}}\left(V_{B}\right)\right)-\operatorname{dim}_{\mathbb{F}} \operatorname{gr}^{m}\left(D_{\mathrm{dR}}\left(V_{B}\right)\right) .
\end{aligned}
$$

Since $y$ is a closed point of $\mathcal{L}[1 / p]$, this is the dimension of $\mathcal{O}_{\mathcal{L}, y}=\left(\mathcal{O}_{\mathcal{L}, x}\right)_{\mathfrak{q}}$. From Lemma 4.4.2, we deduce

$$
\operatorname{dim}_{\mathbb{F}} \mathcal{O}_{\mathcal{L}, x}(\mathbb{F}[\epsilon]) \leqslant \operatorname{dim} \mathcal{O}_{\mathcal{L}, y}
$$

On the other hand,

$$
\operatorname{dim} \mathcal{O}_{\mathcal{L}, y} \leqslant \operatorname{dim} \mathcal{O}_{\mathcal{L}, x}-1 \leqslant \operatorname{dim} \mathcal{O}_{\mathcal{L}, x} / p
$$

We have used Lemma 4.1.1 for the first inequality and [28, Tag 00OM] for the second. Hence $\mathcal{O}_{\mathcal{L}, x} / p$ is regular and these two displayed inequalities are equalities.

To show $\mathcal{O}_{\mathcal{L}, x}$ is $\mathbb{Z}_{p}$-flat, note that $p$ is in the maximal ideal of $\mathcal{O}_{\mathcal{L}, x}$, and so $\operatorname{dim} \mathcal{O}_{\mathcal{L}, x}[1 / p] \leqslant \operatorname{dim} \mathcal{O}_{\mathcal{L}, x}-1=\operatorname{dim} \mathcal{O}_{\mathcal{L}, y}$. As $\mathcal{O}_{\mathcal{L}, y}$ is obtained from $\mathcal{O}_{\mathcal{L}, x}[1 / p]$ by localization $\operatorname{dim} \mathcal{O}_{\mathcal{L}, y} \leqslant \operatorname{dim} \mathcal{O}_{\mathcal{L}, x}[1 / p]$ and we have equality. Let $I \subset \mathcal{O}_{\mathcal{L}, x}$ be the ideal of elements killed by a power of $p$. Then $\mathcal{O}_{\mathcal{L}, x}[1 / p]=\left(\mathcal{O}_{\mathcal{L}, x} / I\right)[1 / p]$ and so

$$
\begin{aligned}
\operatorname{dim} \mathcal{O}_{\mathcal{L}, x} / p & =\operatorname{dim} \mathcal{O}_{\mathcal{L}, x}-1=\operatorname{dim} \mathcal{O}_{\mathcal{L}, x}\left[\frac{1}{p}\right] \\
& \leqslant \operatorname{dim} \mathcal{O}_{\mathcal{L}, x} / I-1 \leqslant \operatorname{dim} \mathcal{O}_{\mathcal{L}, x} /(I, p) .
\end{aligned}
$$

We conclude $\mathcal{O}_{\mathcal{L}, x} / p$ and $\mathcal{O}_{\mathcal{L}, x} /(I, p)$ have the same dimension. Since $\mathcal{O}_{\mathcal{L}, x} / p$ is regular, the image of $I$ in $\mathcal{O}_{\mathcal{L}, x} / p$ must be zero, and so $I \subset p \mathcal{O}_{\mathcal{L}, x}$. As such, any $x \in I$ can be written as $x=p y$; by the definition of $I$, we see $y \in I$ and so $I \subset \cap p^{n} \mathcal{O}_{\mathcal{L}, x}=0$. We conclude $\mathcal{O}_{\mathcal{L}, x}$ is $\mathbb{Z}_{p}$-flat. That the completion of $\mathcal{O}_{\mathcal{L}, x}$ at its maximal ideal is a power series ring is then a standard consequence of the fact that $\mathcal{O}_{\mathcal{L}, x}$ is $\mathbb{Z}_{p}$-flat and $\mathcal{O}_{\mathcal{L}, x} / p$ is regular.

COROLlaRY 4.4.5. Assume that $V_{\mathbb{F}}$ is cyclotomic-free and that $K_{\infty} \cap K\left(\mu_{p^{\infty}}\right)=$ $K$ if $p=2$. The closed subscheme of $\mathcal{L}$ defined by the ideal of p-power torsion sections is equal to a union of connected components of $\mathcal{L}$, and is regular. This closed subscheme therefore coincides with $\mathcal{L}^{\circ}$ defined in Lemma 4.2.6.

Proof. Since $V_{\mathbb{F}}$ is cyclotomic-free, Proposition 4.4.4 implies that a closed point $x \in \mathcal{L}$ is contained in $\mathcal{L}^{\circ}$ if and only if $\mathcal{O}_{\mathcal{L}, x}$ is $\mathbb{Z}_{p}$-flat. Further, if this is the case, then $\mathcal{O}_{\mathcal{L}, x}$ is regular. Flatness implies $\mathcal{L}^{\circ} \subset \mathcal{L}$ is open; see [28, Tag 00RC]. We see $\mathcal{L}^{\circ}$ is regular as it is regular at closed points. 


\section{Applications to deformation rings}

\subsection{Potential diagonalizability}

5.1.1. Now consider a finite extension $E$ of $\mathbb{Q}_{p}$, with residue field $\mathbb{F}$, and a finite free $\mathcal{O}_{E}$-module $V$ equipped with a continuous $\mathcal{O}_{E}$-linear action of $G_{K}$. Assume that $V[1 / p]$ is crystalline of $p$-adic Hodge type $\mathbf{v}$. Let $V_{\mathbb{F}}=V \otimes_{\mathcal{O}_{E}} \mathbb{F}$ and let $R_{\text {crys }}^{\mathbf{v}}$ denote the quotient of $R_{V_{\mathbb{F}}}^{\square}$ from Proposition 4.2.12 (or more generally, the quotient defined in [21] if $\mathbf{v}$ is not concentrated in degrees $[0, p])$. If $\xi$ is an $\mathcal{O}_{E}$-basis of $V$, we say $(V, \xi)$ is diagonalizable if the corresponding point of $R_{\text {crys }}^{\mathbf{v}}$ lies on the same irreducible component of $R_{\text {crys }}^{\mathbf{v}}$ as an $\mathcal{O}_{\bar{E}}$-valued point (here $\mathcal{O} \bar{E}$ denotes the ring of integers in an algebraic closure of $E$ ) whose corresponding representation is a direct sum of crystalline characters. (Since $R_{\text {crys }}^{\mathbf{v}}$ is $\mathbb{Z}_{p}$-flat, this is equivalent to asking that the image of their generic fibres lie on the same irreducible component of $R_{\text {crys }}^{\mathbf{v}}[1 / p]$.) Say $V$ is potentially diagonalizable if $\left.V\right|_{G_{L}}$ is diagonalizable for some finite extension $L$ of $K$. These notions were introduced in $[1,1.4]$.

LEMMA 5.1.2. (1) Whether or not $V$ is potentially diagonalizable is independent of the choice of $\xi$.

(2) If $V^{\prime}$ is a $G_{K}$-stable $\mathcal{O}_{E}$-lattice inside $V[1 / p]$, then $V$ is potentially diagonalizable if and only if $V^{\prime}$ is.

(3) If $V[1 / p]$ admits $a G_{K}$-stable filtration, then $V$ is potentially diagonalizable if and only if each graded piece is potentially diagonalizable. In particular, this is the case if each graded piece is one-dimensional.

Proof. Both (1) and (2) follow from [1, 1.4.1]. For (3), we refer the reader to $[13,2.1 .2]$.

We now prove Theorem 1.1.1. Recall the definition of strongly cyclotomic-free is given in Definition 4.3.7.

THEOREM 5.1.3. Assume $\mathbb{F}$ is sufficiently large and that the p-adic Hodge type $\mathbf{v}$ is concentrated in degree $[0, p]$. If $V_{\mathbb{F}}$ is strongly cyclotomic-free, then $V$ is potentially diagonalizable.

Proof. First, if $p=2$, then we choose our compatible system of $p$ th power roots of a uniformizer of $K$ so that $K_{\infty} \cap K\left(\mu_{p^{\infty}}\right)=K$. This can always be done; see [29, Lemma 2]. As $V_{\mathbb{F}}$ is strongly cyclotomic-free, $\left.V_{\mathbb{F}}\right|_{G_{L}}$ is strongly cyclotomicfree for any finite unramified extension $L / K$. As $\mathbb{F}$ is sufficiently large, we may 
therefore assume each Jordan-Holder factor of $V_{\mathbb{F}}$ is one-dimensional. Under these assumptions, we claim there exists another deformation $V^{\prime}$ of $V_{\mathbb{F}}$ such that:

- $V^{\prime}[1 / p]$ is crystalline with Hodge-Tate weights in $[0, p]$.

- Every Jordan-Holder factor of $V^{\prime}[1 / p]$ is one-dimensional.

- If $\mathfrak{M}$ and $\mathfrak{M}^{\prime}$ are the Breuil-Kisin modules associated with $V$ and $V^{\prime}$, respectively, then $\mathfrak{M} \otimes_{\mathcal{O}_{E}} \mathbb{F}=\mathfrak{M}^{\prime} \otimes_{\mathcal{O}_{E}} \mathbb{F}$ in $\mathcal{L}_{\text {crys }}^{\leqslant p}\left(V_{\mathbb{F}}\right)$.

Such a $V^{\prime}$ is constructed below; see Corollary 5.1.6.

Assuming for now that $V^{\prime}$ can be constructed, we explain how this implies potential diagonalizability of $V$. Let $\mathcal{L}=\mathcal{L}_{R \text {,crys }}^{\leqslant p}$ and $\mathcal{L}^{\circ} \subset \mathcal{L}$ be the $\mathbb{Z}_{p}$-flat locus from Corollary 4.4.5. Then it follows that $\mathfrak{M}$ and $\mathfrak{M}^{\prime}$ induce $\mathcal{O}_{E}$-valued points of $\mathcal{L}^{\circ}$ (see (2) of Remark 2.2.16). The image of the closed point in $\operatorname{Spec} \mathcal{O}_{E}$ under these two maps coincide, and so both $\mathcal{O}_{E}$-points lie on the same connected component of $\mathcal{L}^{\circ}$. By Corollary 4.4.5, $\mathcal{L}^{\circ}$ is normal, and so these points lie on the same irreducible component. Hence their images in $R_{\text {crys }}^{\mathbf{v}}$ lie in the same irreducible component. By (3) of Lemma 5.1.2, we know $V^{\prime}$ is potentially diagonalizable, and so $V$ is potentially diagonalizable also.

To complete the proof of Theorem 5.1.3, we must construct a $V^{\prime}$ as above. Thus we make the following definition.

Definition 5.1.4. Let $\mathfrak{M}_{\mathbb{F}} \in \mathcal{L} \leqslant p\left(V_{\mathbb{F}}\right)$. We say $\mathfrak{M}_{\mathbb{F}}$ admits a crystalline lift if there exists a finite extension $E / \mathbb{Q}_{p}$ with residue field $\mathbb{F}^{\prime}$ containing $\mathbb{F}$ and a finite free $\mathcal{O}_{E}$-module $V$ equipped with a continuous $\mathcal{O}_{E}$-linear action of $G_{K}$ so that (i) $V[1 / p]$ is crystalline with Hodge-Tate weights in $[0, p]$, (ii) $V \otimes_{\mathcal{O}_{E}} \mathbb{F}^{\prime}=$ $V_{\mathbb{F}} \otimes_{\mathbb{F}} \mathbb{F}^{\prime}$, and (iii) if $\mathfrak{M}$ denotes the Breuil-Kisin module associated with $V$, then $\mathfrak{M} \otimes_{\mathcal{O}_{E}} \mathbb{F}^{\prime}=\mathfrak{M}_{\mathbb{F}} \otimes_{\mathbb{F}} \mathbb{F}^{\prime}$ in $\mathcal{L}^{\leqslant p}\left(V_{\mathbb{F}^{\prime}}\right)$.

For the next lemma, consider a $G_{K_{\infty}}$-equivariant exact sequence $0 \rightarrow W_{\mathbb{F}} \rightarrow$ $V_{\mathbb{F}} \rightarrow Z_{\mathbb{F}} \rightarrow 0$ of finite-dimensional $\mathbb{F}$-vector spaces. If $\mathfrak{M}_{\mathbb{F}} \in \mathcal{L} \leqslant p\left(V_{\mathbb{F}}\right)$, then Lemma 3.2.5 produces an exact sequence $0 \rightarrow \mathfrak{W}_{\mathbb{F}} \rightarrow \mathfrak{M}_{\mathbb{F}} \rightarrow \mathfrak{Z}_{\mathbb{F}} \rightarrow 0$.

LEMma 5.1.5. Assume that $\mathfrak{W} \in \mathcal{L}_{\text {crys }}^{\leqslant p}(W)$ and $\mathfrak{Z} \in \mathcal{L}_{\text {crys }}^{\leqslant p}(Z)$ are crystalline lifts of $W_{\mathbb{F}}$ and $Z_{\mathbb{F}}$, respectively. Assume that $\mathfrak{W}_{\mathbb{F}} \oplus \mathfrak{Z}_{\mathbb{F}}$ satisfies (2) of Hypothesis 4.3.2, and that $\mathfrak{M}_{\mathbb{F}}$ from the previous paragraph is strongly divisible. Then there exists a crystalline lift $\mathfrak{M} \in \mathcal{L}_{\text {crys }}^{\leqslant p}(V)$ of $\mathfrak{M}_{\mathbb{F}}$ such that:

(1) $\mathfrak{M}$ fits into a $\varphi$-equivariant exact sequence $0 \rightarrow \mathfrak{W} \rightarrow \mathfrak{M} \rightarrow \mathfrak{Z} \rightarrow 0$ of $\mathfrak{S}_{\mathcal{O}_{E}}$-modules, which recovers $0 \rightarrow \mathfrak{W}_{\mathbb{F}} \rightarrow \mathfrak{M}_{\mathbb{F}} \rightarrow \mathfrak{Z}_{\mathbb{F}} \rightarrow 0$ after applying base-changing to $\mathbb{F}^{\prime}$. 
(2) $V$ fits into a $G_{K}$-equivariant exact sequence $0 \rightarrow W \rightarrow V \rightarrow Z \rightarrow 0$, which recovers $0 \rightarrow \mathfrak{W} \rightarrow \mathfrak{M} \rightarrow \mathfrak{Z} \rightarrow 0$ after base-changing to $W\left(C^{b}\right)$.

In particular, if both $Z_{\mathbb{F}}$ and $W_{\mathbb{F}}$ are cyclotomic-free, then $Z_{\mathbb{F}} \oplus W_{\mathbb{F}}$ is cyclotomic-free also, and so in this case (1) of Hypothesis 4.3.2 is satisfied for $\mathfrak{W}_{\mathbb{F}} \oplus \mathfrak{Z}_{\mathbb{F}}$. We remark also that a similar result is proven $[3,5.3 .1]$ but with a different notion of cyclotomic-free.

Proof. The element $\mathfrak{W}_{\mathbb{F}} \oplus \mathfrak{Z}_{\mathbb{F}} \in \mathcal{L}_{\text {crys }}^{\leqslant p}\left(W_{\mathbb{F}} \oplus Z_{\mathbb{F}}\right)$ defines a closed point $x$ of the $R=R_{W_{\mathbb{F}} \oplus Z_{\mathbb{F}}}^{\square}$-scheme $\mathcal{L}=\mathcal{L}_{R \text {,crys }}^{\leqslant p}$ from Corollary 2.2.11. Also $\mathfrak{W} \oplus \mathfrak{Z}$ defines an $\mathcal{O}_{E}$-valued point $y$ of $\mathcal{L}$ through which $x$ factors.

View the extension $0 \rightarrow \mathfrak{W}_{\mathbb{F}} \rightarrow \mathfrak{M}_{\mathbb{F}} \rightarrow \mathfrak{Z}_{\mathbb{F}} \rightarrow 0$ as an extension of $\mathfrak{W}_{\mathbb{F}} \oplus \mathfrak{Z}_{\mathbb{F}}$ by itself. By assumption, $\mathfrak{M}_{\mathbb{F}}$ is strongly divisible, so we obtain an element of the set $\operatorname{Ext}_{\mathrm{SD}}^{1}\left(\mathfrak{W}_{\mathbb{F}} \oplus \mathfrak{Z}_{\mathbb{F}}, \mathfrak{W}_{\mathbb{F}} \oplus \mathfrak{Z}_{\mathbb{F}}\right)$ described in the proof of Lemma 4.4.2. It follows from the proof of Proposition 4.4.4 that the map $\mathcal{O}_{\mathcal{L}, x}(\mathbb{F}[\epsilon]) \rightarrow \operatorname{Ext}_{\mathrm{SD}}^{1}\left(\mathfrak{W}_{\mathbb{F}} \oplus \mathfrak{Z}_{\mathbb{F}}\right.$, $\left.\mathfrak{W}_{\mathbb{F}} \oplus \mathfrak{Z}_{\mathbb{F}}\right)$ in (4.4.3) is surjective. Thus there is a tangent vector $x^{\prime}: \operatorname{Spec} \mathbb{F}[\epsilon] \rightarrow \mathcal{L}$ mapping onto this extension class. Since the completion of $\mathcal{O}_{\mathcal{L}, x}$ is a power series ring over $W(\mathbb{F})$, the point $y$ factors through a morphism $\operatorname{Spec} \mathcal{O}_{E}[\epsilon] \rightarrow \mathcal{L}$ lifting $x^{\prime}$ (here $\mathcal{O}_{E}[\epsilon]$ denotes the ring of dual numbers over $\mathcal{O}_{E}$ ). This morphism induces an extension $0 \rightarrow W \oplus Z \rightarrow V^{\prime} \rightarrow W \oplus Z \rightarrow 0$ as well as an $\mathfrak{M}^{\prime} \in \mathcal{L}_{\text {crys }}^{\leqslant p}\left(V^{\prime}\right)$ fitting into an exact sequence

$$
0 \rightarrow \mathfrak{W} \oplus \mathfrak{Z} \rightarrow \mathfrak{M}^{\prime} \rightarrow \mathfrak{W} \oplus \mathfrak{Z} \rightarrow 0 .
$$

From this, we obtain the representation $V$ and $\mathfrak{M} \in \mathcal{L}_{\text {crys }}^{\leqslant p}(V)$ as desired.

COROLlary 5.1.6. Suppose $V_{\mathbb{F}}$ is cyclotomic-free and every Jordan-Holder factor is one-dimensional. Let $\mathfrak{M}_{\mathbb{F}} \in \mathcal{L}_{\mathrm{SD}}^{\leqslant p}\left(V_{\mathbb{F}}\right)$. Then $\mathfrak{M}_{\mathbb{F}}$ admits a crystalline lift $V$ so that every Jordan-Holder factor of $V[1 / p]$ is one-dimensional.

Proof. If $V_{\mathbb{F}}$ is one-dimensional, then the result is easy (see for example part (1) of [16, Lemma 6.3]). For the general case, induct on the length of $V_{\mathbb{F}}$ using Lemma 5.1.5.

In particular, we see $\mathfrak{M} \in \mathcal{L}_{\text {crys }}^{\leqslant p}\left(V_{\mathbb{F}}\right)$.

5.2. A possible improvement. We would now like to explain how Theorem 5.1.3 can be strengthened, assuming a conjectural statement regarding the fibre of $\mathcal{L}$ over the closed point of $\operatorname{Spec} R$.

5.2.1. As usual, let $\mathbb{F}$ denote a finite field of characteristic $p$ and let $V_{\mathbb{F}}$ denote a finite-dimensional $\mathbb{F}$-vector space equipped with a continuous $\mathbb{F}$-linear action 
of $G_{K}$. Let $R=R_{V_{\mathbb{F}}}^{\square}$ and $\mathcal{L}=\mathcal{L}_{R \text {, crys }}^{\leqslant p}$. We also let $\mathcal{L}_{\mathbb{F}}=\mathcal{L} \otimes_{R} \mathbb{F}$ be the fibre of $\mathcal{L}$ over the closed point of Spec $R$.

5.2.2. Let us first assume $V_{\mathbb{F}}$ is absolutely irreducible and $\mathbb{F}$ is sufficiently large, so that $V_{\mathbb{F}}=\operatorname{Ind}_{G_{L}}^{G_{K}} W_{\mathbb{F}}$ with $L / K$ unramified and $W_{\mathbb{F}}$ one-dimensional. We also assume that the residue field $l$ of $L$ embeds into $\mathbb{F}$. Recall from Lemma 3.1.8 that there is a map $f_{*}: \mathcal{L}^{\leqslant p}\left(W_{\mathbb{F}}\right) \rightarrow \mathcal{L}^{\leqslant p}\left(V_{\mathbb{F}}\right)$. It follows from 3.2.9 and Lemma 3.2.11 that the image of this map lies in $\mathcal{L}_{\text {crys }}^{\leqslant p}\left(V_{\mathbb{F}}\right)$.

CONJECTURE 5.2.3. With $V_{\mathbb{F}}$ as in 5.2.2, every closed point of $\mathcal{L}_{\mathbb{F}}$ lies in the same connected component as a closed point arising from $f_{*} \mathfrak{N}$ for some $\mathfrak{N} \in \mathcal{L} \leqslant p\left(W_{\mathbb{F}}\right)$.

We are going to prove this when $V_{\mathbb{F}}$ is 2-dimensional. Before doing so, we record some consequences of this conjecture.

Lemma 5.2.4. Suppose Conjecture 5.2.3 holds and, if $p=2$, that $K_{\infty} \cap$ $K\left(\mu_{p^{\infty}}\right)=K$. Then any $\mathfrak{M} \in \mathcal{L}_{\text {crys }}^{\leqslant p}\left(V_{\mathbb{F}}\right)$ admits a crystalline lift.

In particular, we deduce $\mathcal{L}^{\circ}=\mathcal{L}$ in this situation.

Proof. We have to show the local ring of $\mathcal{L}$ at the closed point corresponding to $\mathfrak{M}$ is nonzero after inverting $p$. After Corollary 4.4.5, it suffices to show every connected component of $\mathcal{L}$ contains at least one closed point admitting a crystalline lift. Using the conjecture, we are reduced to proving that, if $\mathfrak{N} \in \mathcal{L}^{\leqslant p}\left(W_{\mathbb{F}}\right), f_{*} \mathfrak{N}$ admits a crystalline lift, and this is easy. Choose a crystalline character lifting $\mathfrak{N}$ and consider the induction of that character from $G_{L}$ to $G_{K}$.

Lemma 5.2.5. Suppose Conjecture 5.2.3 for all absolutely irreducible $V_{\mathbb{F}}$ and, if $p=2$, that $K_{\infty} \cap K\left(\mu_{p^{\infty}}\right)=K$. Then any $\mathfrak{M} \in \mathcal{L}_{\mathrm{SD}}^{\leqslant p}\left(V_{\mathbb{F}}^{\prime}\right)$ with $V_{\mathbb{F}}^{\prime}$ cyclotomicfree (but not necessarily irreducible) admits a crystalline lift $V$ such that every Jordan-Holder factor of $V[1 / p]$ has irreducible reduction modulo $p$.

Proof. Using Lemma 5.2.4, this follows as in Corollary 5.1.6 by inductively applying Lemma 5.1.5.

COROLlary 5.2.6. Suppose Conjecture 5.2.3 holds for all absolutely irreducible $V_{\mathbb{F}}$. Then Theorem 5.1 .3 holds with strong cyclotomic freeness replaced by cyclotomic freeness.

Proof. First, suppose the $V \otimes_{\mathcal{O}_{E}} \mathbb{F}=V_{\mathbb{F}}$ is irreducible. Conjecture 5.2.3 then implies $V$ lies in the same irreducible component of $R_{\text {crys }}^{\mathbf{v}}$ as a point obtained by 
inducing a crystalline character over an unramified extension. Since such points are potentially diagonalizable (after a finite extension, they become a sum of crystalline characters), this proves the result in the irreducible case.

For the general case, we know after Lemma 5.2.5 that $V$ lies in the same component as a point whose Jordan-Holder factors are all irreducible modulo $p$. The previous paragraph implies each of these Jordan-Holder factors is potentially diagonalizable, and so Lemma 5.1.2 implies the point itself is potentially diagonalizable. We conclude $V$ is also.

\section{Proposition 5.2.7. Conjecture 5.2.3 holds if $V_{\mathbb{F}}$ is two-dimensional.}

Proof. Replacing $V_{\mathbb{F}}$ by an unramified twist (which is allowable by Lemma 3.1.5 and the comment made in 3.1.7), we can assume the situation is as in the proof of Proposition 3.2.2; see in particular 3.2.4 and 3.2.6. Thus there is an $\mathfrak{N} \in \mathcal{L} \leqslant p\left(W_{\mathbb{F}}\right)$ with generators $\left(e_{\theta}\right)_{\theta \in \operatorname{Hom}_{\mathbb{F}_{p}}(l, \mathbb{F})}$ satisfying

$$
\varphi\left(e_{\theta \circ \varphi}\right)=u^{r_{\theta}} e_{\theta}, \quad 0 \leqslant r_{\theta} \leqslant p
$$

together with an inclusion $\mathfrak{M} \subset f_{*} \mathfrak{N}$ (where $f_{*} \mathfrak{N}$ denotes $\mathfrak{N}$ viewed as an $\mathfrak{S}_{\mathbb{F}}$-module). For $\mathfrak{M}$ to be contained in $\mathcal{L}_{\text {crys }}^{\leqslant p}\left(V_{\mathbb{F}}\right)$, it is necessary and sufficient that:

- If $m \in \mathfrak{M}$, then $\varphi(m) \in \mathfrak{M}$. If $\varphi(m) \in u^{p+1} \mathfrak{M}$, then $m \in u \mathfrak{M}$.

- If $\sum \alpha_{\theta} e_{\theta} \in \mathfrak{M}$ with $\alpha_{\theta} \in \mathbb{F}$, then $\sum_{r_{\theta} \equiv r \bmod p} \alpha_{\theta} e_{\theta} \in \mathfrak{M}$.

(see 3.2.8). Recall that the first condition is implied by $u^{p} \mathfrak{M} \subset \mathfrak{M}^{\varphi} \subset \mathfrak{M}$, and it implies $u e_{\theta} \in \mathfrak{M}$ for every $\theta$.

For $\tau \in \operatorname{Hom}_{\mathbb{F}_{p}}(k, \mathbb{F})$, we write $\mathfrak{M}_{\tau}$ for the summand of $\mathfrak{M}$ on which $k$ acts through $\tau$ (with $\mathfrak{M}$ viewed as an $\mathbb{F}[[u]]$-module). If $\theta \in \operatorname{Hom}_{\mathbb{F}_{p}}(l, \mathbb{F})$ is such that $\left.\theta\right|_{k}=\tau$, then elements of $\mathfrak{M}_{\tau}$ have the form $\alpha e_{\theta}+\beta e_{\theta \circ \varphi^{h}}$, where $h=\left[K: \mathbb{Q}_{p}\right]$. In particular, the possible shapes of the $\mathfrak{M}_{\tau}$ can be divided into two:

(1) Either there is an $\alpha \in \mathbb{F}^{\times}$so that $e_{\theta}+\alpha e_{\theta \circ \varphi^{h}}$ and $u e_{\theta}$ generate $\mathfrak{M}_{\tau}$ over $\mathbb{F}[[u]]$ (for some $\theta$ with $\left.\theta\right|_{k}=\tau$ ).

(2) Or no such $\alpha$ exists. Thus $\mathfrak{M}_{\tau}$ is generated by $u^{x_{\theta}} e_{\theta}$ and $u^{x_{\theta \circ \varphi^{h}}} e_{\theta \circ \varphi^{h}}$ for some $x_{\theta}, x_{\theta \circ \varphi^{h}} \in[0,1]$ (again $\theta$ is some embedding with $\left.\theta\right|_{k}=\tau$ ).

Set $d(\mathfrak{M})$ equal to the number of $\tau$ as in case (i). Note that if $d(\mathfrak{M})=0$, then $\mathfrak{M}=f_{*} \mathfrak{N}^{\prime}$, where $\mathfrak{N}^{\prime} \in \mathcal{L}^{\leqslant p}\left(W_{\mathbb{F}}\right)$ is the $\mathfrak{S}_{\mathbb{F}}$-submodule of $\mathfrak{N}$ generated by $u^{x_{\theta}} \boldsymbol{e}_{\theta}$. Arguing by induction, it therefore suffices to show $\mathfrak{M}$ lies in the same connected component of $\mathcal{L}_{\mathbb{F}}$ as an $\mathfrak{M}^{\prime} \subset f_{*} \mathfrak{N}$ with $d\left(\mathfrak{M}^{\prime}\right)<d(\mathfrak{M})$. For this, we will need a lemma. 
LeMma 5.2.8. Suppose $\mathfrak{M}_{\tau}$ and $\mathfrak{M}_{\tau \circ \varphi}$ are as in (i) and $\theta \in \operatorname{Hom}_{\mathbb{F}_{p}}(l, \mathbb{F})$ with $\left.\theta\right|_{k}=\tau$. Then $\mathfrak{M}_{\tau}$ is generated by $e_{\theta}+\alpha e_{\theta \circ \varphi^{h}}$ and $u e_{\theta}$, for some $\alpha \in \mathbb{F}^{\times}$, if and only if $\mathfrak{M}_{\tau \circ \varphi}$ is generated by $e_{\theta \circ \varphi}+\alpha e_{\theta \circ \varphi^{h+1}}$ and $u e_{\theta \circ \varphi}$. Furthermore, $r_{\theta}=r_{\theta \circ \varphi^{h}}$.

Proof. The second bullet point above implies $r_{\theta} \equiv r_{\theta \circ \varphi^{h}}$ modulo $p$, so we have equality except possibly if $r_{\theta}=0$ or $p$. Suppose $r_{\theta}=0$ so that $r_{\theta \circ \varphi^{h}}$ equals 0 or $p$. As such, if $e_{\theta \circ \varphi}+\alpha e_{\theta \circ \varphi^{h+1}} \in \mathfrak{M}_{\tau \circ \varphi}$, then applying $\varphi$ shows that $e_{\theta}+\alpha u^{r_{\theta \circ \varphi^{h}}} e_{\theta \circ \varphi^{h}} \in$ $\mathfrak{M}_{\tau}$. If $r_{\theta \circ \varphi^{h}}=p$, then $e_{\theta} \in \mathfrak{M}_{\tau}$, which contradicts the fact that $\mathfrak{M}_{\tau}$ is as in (i). Thus $r_{\theta \circ \varphi^{h}}=0$ and $e_{\theta}+\alpha e_{\theta \circ \varphi^{h}} \in \mathfrak{M}_{\tau}$. This proves the lemma when $r_{\theta}=0$.

Now suppose $r_{\theta}>0$. Then $r_{\theta \circ \varphi^{h}}=r_{\theta}$ except possibly if $r_{\theta}=p$ and $r_{\theta \circ \varphi^{h}}=0$. Applying the previous paragraph with $\theta$ replaced by $\theta \circ \varphi^{h}$ shows the exceptional case is impossible. Suppose $\alpha \in \mathbb{F}$ is such that $e_{\theta}+\alpha e_{\theta \circ \varphi^{h}} \in \mathfrak{M}_{\tau}$. The first bullet point above implies that $e_{\theta \circ \varphi}+\alpha e_{\theta \circ \varphi^{h+1}} \in \mathfrak{M}_{\tau \circ \varphi}$ (since $\varphi$ maps $u\left(e_{\theta \circ \varphi}+\alpha e_{\theta \circ \varphi^{h+1}}\right)$ onto $\left.u^{p+r_{\theta}}\left(e_{\theta}+\alpha e_{\theta \circ \varphi^{h}}\right)\right)$. This proves the lemma when $r_{\theta}>0$.

If $M_{\mathbb{F}}$ is the etale $\varphi$-module associated with $V_{\mathbb{F}}$, set $M_{\mathbb{F}[T]}=M_{\mathbb{F}} \otimes_{\mathbb{F}} \mathbb{F}[T]$, for a formal variable $T$. We are going to construct $\mathfrak{M}_{\mathbb{F}[T]} \in \mathcal{L}_{\text {crys }}^{\leqslant p}\left(V_{\mathbb{F}} \bigotimes_{\mathbb{F}} \mathbb{F}[T]\right)$ with $\mathfrak{M}_{\mathbb{F}[T]} \subset\left(f_{*} \mathfrak{N}\right) \otimes_{\mathbb{F}} \mathbb{F}[T]$, which at $T=1$ recovers $\mathfrak{M}$ and which at $T=0$ produces $\mathfrak{M}^{\prime}$ with $d\left(\mathfrak{M}^{\prime}\right)<d(\mathfrak{M})$. As $\mathfrak{M}_{\mathbb{F}[T]}$ induces a morphism $\mathbb{A}^{1} \rightarrow \mathcal{L}_{\mathbb{F}}$ connecting $\mathfrak{M}$ and $\mathfrak{M}^{\prime}$, this will complete the proof.

To produce $\mathfrak{M}_{\mathbb{F}[T]}$, choose $\tau \in \operatorname{Hom}_{\mathbb{F}_{p}}(k, \mathbb{F})$ so that $J=\left\{\tau \circ \varphi^{n}, \tau \circ \varphi^{n-1}, \ldots\right.$, $\tau$ \} is such that $\mathfrak{M}_{\tau \circ \varphi^{j}}$ is as in (i) for $0 \leqslant j \leqslant n$ and as in (ii) for $j=-1$ and $n+1$. We can assume such a $\tau$ exists for the following reasons. We can always assume there is a $\tau$ with $\mathfrak{M}_{\tau}$ as in (i) as otherwise $d(\mathfrak{M})=0$. If $\mathfrak{M}_{\tau}$ is as in (i) for every $\tau$, then Lemma 5.2.8 would imply $r_{\theta}=r_{\theta \circ \varphi^{h}}$ for every $\theta \in \operatorname{Hom}_{\mathbb{F}_{p}}(l, \mathbb{F})$. In this case, the submodule of $f_{*} \mathfrak{N}$ generated by the $e_{\theta}+e_{\theta \circ \varphi^{h}}$ for all $\theta \in \operatorname{Hom}_{\mathbb{F}_{p}}(l, \mathbb{F})$ is $\varphi$-stable and so corresponds to a $G_{K_{\infty}}$-subrepresentation of $V_{\mathbb{F}}$, contradicting irreducibility.

Choose $\theta \in \operatorname{Hom}_{\mathbb{F}_{p}}(l, \mathbb{F})$ so that $\left.\theta\right|_{k}=\tau$; there is an $\alpha \in \mathbb{F}^{\times}$such that $\mathfrak{M}_{\tau}$ is generated over $\mathbb{F}[[u]]$ by $e_{\theta}+\alpha e_{\theta \circ \varphi^{h}}$ and $u e_{\theta}$. Using Lemma 5.2.8, we see $\mathfrak{M}_{\tau \circ \varphi^{j}}$ is generated by $e_{\theta \circ \varphi^{j}}+\alpha e_{\theta \circ \varphi^{h+j}}$ and $u e_{\theta \circ \varphi^{h+j}}$ for $0 \leqslant j \leqslant n$. For $0 \leqslant j \leqslant n$, we define

$$
\mathfrak{M}_{\mathbb{F}[T], \tau \circ \varphi^{j}}=\text { the } \mathbb{F}[[u]][T] \text {-linear span of } e_{\theta \circ \varphi^{j}}+T \alpha e_{\theta \circ \varphi^{h+j}} \text { and } u e_{\theta \circ \varphi^{j}}
$$

(note that this is well defined; for $0 \leqslant j, j^{\prime} \leqslant n$, if $\tau \circ \varphi^{j}=\tau \circ \varphi^{j^{\prime}}$, then $j=j^{\prime}$; otherwise, $J=\operatorname{Hom}_{\mathbb{F}_{p}}(k, \mathbb{F})$, which we have shown in the paragraph above is impossible). For $\tau^{\prime} \notin J$, we set $\mathfrak{M}_{\mathbb{F}[T], \tau^{\prime}}=\mathfrak{M}_{\tau^{\prime}} \otimes_{\mathbb{F}} \mathbb{F}[T]$. Define $\mathfrak{M}_{\mathbb{F}[T]}=$ $\bigoplus_{\tau^{\prime} \in \operatorname{Hom}_{\mathbb{F}}(k, \mathbb{F})} \mathfrak{M}_{\mathbb{F}[T], \tau^{\prime}}$. This is a projective $\mathfrak{S}_{\mathbb{F}[T]}$-module inside $M_{\mathbb{F}[T]}$, which by construction equals $\mathfrak{M}$ at $T=1$. The scheme from Proposition 2.2.3 is described as a closed subscheme of the affine Grassmannian, and so $\mathcal{L}_{\mathbb{F}}$ is also closed in 
the affine Grassmannian. Thus to show $\mathfrak{M}_{\mathbb{F}[T]} \in \mathcal{L}_{\text {crys }}^{\leqslant p}\left(V_{\mathbb{F}} \otimes_{\mathbb{F}} \mathbb{F}[T]\right)$, it suffices to show that $\mathfrak{M}_{\lambda} \in \mathcal{L}_{\text {crys }}^{\leqslant p}\left(V_{\mathbb{F}} \otimes_{\mathbb{F}} \mathbb{F}^{\prime}\right)$ whenever $\mathbb{F}^{\prime}$ is a finite extension of $\mathbb{F}$ and $\mathfrak{M}_{\lambda}$ is obtained from $\mathfrak{M}_{\mathbb{F}[T]}$ by evaluating $T$ at $\lambda \in \mathbb{F}^{\prime}$. This means verifying the two bullet points above for $\mathfrak{M}_{\lambda}$. The second bullet point is clear from the construction of $\mathfrak{M}_{\mathbb{F}[T]}$. To show the first, we only have to check $u^{p} \mathfrak{M}_{\lambda} \subset \mathfrak{M}_{\lambda}^{\varphi} \subset \mathfrak{M}_{\lambda}$. If $\tau^{\prime}$ is such that both $\tau^{\prime}$ and $\tau^{\prime} \circ \varphi$ are not in $J$, then

$$
u^{p} \mathfrak{M}_{\lambda, \tau^{\prime}} \subset \mathfrak{M}_{\lambda, \tau^{\prime}}^{\varphi} \subset \mathfrak{M}_{\lambda, \tau^{\prime}}
$$

since $\mathfrak{M}_{\lambda, \tau^{\prime} \circ \varphi}=\mathfrak{M}_{\tau^{\prime} \circ \varphi}$ (so that $\mathfrak{M}_{\lambda, \tau^{\prime}}^{\varphi}=\mathfrak{M}_{\tau^{\prime}}^{\varphi}$ ) and $\mathfrak{M}_{\lambda, \tau^{\prime}}=\mathfrak{M}_{\tau^{\prime}}$. For the remaining $\tau^{\prime}$, we choose $\mathbb{F}[[u]]$-bases $\xi_{\tau^{\prime}}$ of $\mathfrak{M}_{\lambda, \tau^{\prime}}$ as follows:

(1) If $\tau^{\prime}=\tau \circ \varphi^{j} \in J$, so that $0 \leqslant j \leqslant n$, then $\xi_{\tau^{\prime}}=\left(e_{\theta \circ \varphi^{j}}+\lambda \alpha e_{\theta \circ \varphi^{h+j}}, u e_{\theta \circ \varphi^{h+j}}\right)$.

(2) If $\tau^{\prime}=\tau \circ \varphi^{-1}$ or $\tau \circ \varphi^{n+1}$ not in $J$, then $\xi_{\tau^{\prime}}=\left(u^{\delta_{j}} e_{\theta \circ \varphi^{j}}, u^{\delta_{j+h}} e_{\theta \circ \varphi^{j+h}}\right)$, where $\delta_{j}$ and $\delta_{j+h}$, respectively, equal $x_{\theta \circ \varphi^{j}}$ and $x_{\theta \circ \varphi^{j+h}}$ as defined in (ii) above. In particular, both $\delta_{j}$ and $\delta_{j+h}$ are integers in $[0,1]$.

There are matrices $A_{\tau^{\prime}}$ valued in $\mathbb{F}[[u]]$ so that $\varphi\left(\xi_{\tau^{\prime} \circ \varphi}\right)=\left(\xi_{\tau^{\prime}}\right) A_{\tau^{\prime}}$. If $\tau^{\prime}=\theta \circ \varphi^{j}$ with $\tau^{\prime} \circ \varphi$ and $\tau^{\prime} \in J$ (that is $0 \leqslant j \leqslant n-1$ ), then we compute

$$
A_{\tau^{\prime}}=\left(\begin{array}{cc}
u^{r_{\circ \circ \varphi}} & 0 \\
0 & u^{p-1+r_{\theta \circ \varphi^{h+j}}}
\end{array}\right) .
$$

This does not depend on $\lambda$, so we deduce that $u^{p} \mathfrak{M}_{\lambda, \tau^{\prime}} \subset \mathfrak{M}_{\lambda, \tau^{\prime}}^{\varphi} \subset \mathfrak{M}_{\lambda, \tau^{\prime}}$ from the fact that it holds when $\lambda=1$. If $\tau^{\prime}=\tau$, so $\tau^{\prime} \in J$ but $\tau^{\prime} \circ \varphi^{-1} \notin J$, then we compute

$$
A_{\tau^{\prime}}=\left(\begin{array}{cc}
u^{r_{\theta \circ \varphi^{-1}}-\delta_{-1}} & 0 \\
\lambda \alpha u^{r_{\theta \circ \varphi^{h-1}}-\delta_{h-1}} & u^{p+r_{\theta \circ \varphi^{h-1}}-\delta_{h-1}}
\end{array}\right) .
$$

As such, in order that $u^{p} \mathfrak{M}_{\lambda, \tau^{\prime}} \subset \mathfrak{M}_{\lambda, \tau^{\prime}}^{\varphi} \subset \mathfrak{M}_{\lambda, \tau^{\prime}}$, we need both $r_{\theta \circ \varphi^{-1}}-\delta_{-1}=$ $r_{\theta \circ \varphi^{h-1}}-\delta_{h-1}=0$. Setting $\lambda=1$, we see this is the case, so it is the case for any $\lambda$. Finally we consider the case $\tau^{\prime}=\tau \circ \varphi^{n}$ so that $\tau^{\prime} \circ \varphi \notin J$ and $\tau^{\prime} \in J$. Then

$$
A_{\tau^{\prime}}=\left(\begin{array}{cc}
u^{p \delta_{n+1}+r_{\theta \circ \varphi^{n}}} & 0 \\
-\lambda \alpha u^{p \delta_{n+1}+r_{\theta \circ \varphi^{n}}-1} & u^{p \delta_{n+h+1}+r_{\theta \circ \varphi^{n+h}}-1}
\end{array}\right) .
$$

Again whether or not $u^{p} \mathfrak{M}_{\lambda, \tau^{\prime}} \subset \mathfrak{M}_{\lambda, \tau^{\prime}}^{\varphi} \subset \mathfrak{M}_{\lambda, \tau^{\prime}}$ is a condition on the powers of $u$ appearing in this matrix (we must have $p \delta_{n+1}+r_{\theta \circ \varphi^{n}} \in[1, p]$ and $p \delta_{n+h+1}+$ $\left.r_{\theta \circ \varphi^{n+h}}-1 \in[0, p-1]\right)$. As these conditions hold with $\lambda=1$, they hold for general $\lambda$. 
EXAMPLE 5.2.9. While it seems plausible that the same kind of strategy could be used to give a full proof of Conjecture 5.2.3, in higher dimensions, the situation is more complicated. Below we give an example that illustrates this difficulty. Suppose $K=\mathbb{Q}_{p}$ and that $L / K$ is the unramified extension of degree 7. For an appropriate one-dimensional representation $W_{\mathbb{F}}$ of $G_{L}$, there is an $\mathfrak{N} \in \mathcal{L}^{\leqslant p}\left(W_{\mathbb{F}}\right)$ so that $\mathfrak{N}$ is generated by $\left(e_{6}, e_{5}, \ldots, e_{1}, e_{0}\right)$ with

$$
\varphi\left(e_{6}, \ldots, e_{0}\right)=\left(e_{5}, u e_{4}, e_{3}, u e_{2}, e_{1}, u^{2} e_{0}, u e_{6}\right) .
$$

If $\mathfrak{M} \subset f_{*} \mathfrak{N}$ is the $\mathbb{F}[[u]]$-submodule generated by

$$
e_{6}+e_{4}+e_{2}, e_{5}+e_{3}, u e_{4}, u e_{3}, u e_{2}, e_{1}, e_{0},
$$

then one easily checks that $\mathfrak{M} \in \mathcal{L}_{\text {crys }}^{\leqslant p}\left(V_{\mathbb{F}}\right)$, where $V_{\mathbb{F}}=\operatorname{Ind}_{G_{L}}^{G_{K}} W_{\mathbb{F}}$. If we define $\mathfrak{M}_{\mathbb{F}[T]}$ to be generated over $\mathbb{F}[[u]][T]$ by

$$
e_{6}+T^{2} e_{4}+T e_{2}, e_{5}+T e_{3}, u e_{4}, u e_{3}, u e_{2}, e_{1}, e_{0},
$$

then one also checks by hand that this defines an element of $\mathcal{L}_{\text {crys }}^{\leqslant p}\left(V_{\mathbb{F}} \otimes_{\mathbb{F}} \mathbb{F}[T]\right)$. Thus we obtain a morphism $\mathbb{A}^{1} \rightarrow \mathcal{L}_{\mathbb{F}}$ connecting $\mathfrak{M}$ with $f_{*} \mathfrak{N}^{\prime}$, where $\mathfrak{N}^{\prime} \subset \mathfrak{N}$ is generated by $e_{6}, e_{5}, u e_{4}, u e_{3}, u e_{2}, e_{1}, e_{0}$.

\subsection{Final remarks}

5.3.1. So far we have seen that the scheme $\mathcal{L}^{\circ}$ describes the irreducible components of $R_{\text {crys }}^{\mathrm{v}}$ (they correspond to the connected components of $\mathcal{L}^{\circ}$ ). In some specific situations, we can do better. As usual, let $\mathbb{F}$ be a finite field of characteristic $p$ and $V_{\mathbb{F}}$ a representation of $G_{K}$ on an $\mathbb{F}$-vector space. Assume that $V_{\mathbb{F}}$ is cyclotomic-free and, if $p=2$, that $K_{\infty} \cap K\left(\mu_{p^{\infty}}\right)=K$. We also fix a $p$-adic Hodge type $\mathbf{v}$ concentrated in degree $[0, p]$.

The following notation is taken from [7, 5.1.3]. For complete local Noetherian $\mathbb{Z}_{p}$-algebras $R, R_{1}, \ldots, R_{r}$, write $R \sim \prod_{i=1}^{r} R_{i}$ if there exists a $\mathbb{Z}_{p}$-algebra homomorphism $R \rightarrow \prod R_{i}$, which becomes an isomorphism after inverting $p$ and is such that each projection $R \rightarrow \prod R_{i} \rightarrow R_{j}$ is surjective.

Proposition 5.3.2. Suppose that the fibre of $\mathcal{L}^{\mathbf{v}}$ over the closed point of $\operatorname{Spec} R$ is reduced and zero-dimensional and assume $\mathbb{F}$ is sufficiently large. Then

$$
R_{\text {crys }}^{\mathbf{v}} \sim \prod \mathcal{O}_{\mathcal{L}, x}
$$

where the product runs over the closed points of $\mathcal{L}^{\mathbf{v}}$. (In particular, we are asserting that each $\mathcal{O}_{\mathcal{L}, x}$ is complete.) 
Proof. The fibre of $\mathcal{L}^{\mathbf{v}} \rightarrow$ Spec $R$ over the closed point being zero-dimensional implies this map is quasifinite and therefore finite since it is projective. In particular, $\mathcal{L}^{\mathbf{v}}=\operatorname{Spec} S$ is affine and the induced finite map $R \rightarrow S$ becomes surjective after inverting $p$. By definition, $R_{\text {crys }}^{\mathbf{v}}$ is the quotient of $R$ by the kernel of this map. Clearly, $S$ is a product of the local rings of $\mathcal{L}^{\mathrm{v}}$ at its closed points; all that remains is to show the maps $R_{\text {crys }}^{\mathbf{v}} \rightarrow \mathcal{O}_{\mathcal{L}^{v}, x}$ are surjective. Since $\mathbb{F}$ is assumed to be sufficiently large, we may assume this map is an isomorphism on residue fields, so we only need to show that $\mathfrak{m}_{R_{\text {crys }}^{v}} \mathcal{O}_{\mathcal{L}^{v}, x}=\mathfrak{m}_{\mathcal{L}^{v}, x}$ (that is, that $R_{\text {crys }}^{\mathbf{v}} \rightarrow \mathcal{O}_{\mathcal{L}^{\mathrm{v}}, x}$ is unramified). This follows from the assumption that the fibre of $\mathcal{L}^{\mathbf{v}}$ at the closed point of Spec $R$ is reduced.

5.3.3. We now show how to verify the hypotheses of Proposition 5.3.2 in explicit cases. Let $\mathfrak{M} \in \mathcal{L}_{\text {crys }}^{\leqslant p}\left(V_{\mathbb{F}}\right)$ and suppose $\mathfrak{M}_{\mathbb{F}[\epsilon]} \in \mathcal{L}_{\text {crys }}^{\leqslant p}\left(V_{\mathbb{F}} \otimes_{\mathbb{F}} \mathbb{F}[\epsilon]\right)$ is such that $\mathfrak{M}_{\mathbb{F}[\epsilon]} \otimes_{\mathbb{F}[\epsilon]} \mathbb{F}=\mathfrak{M}$. As in the proof of Lemma 4.4.2, we can view $\mathfrak{M}_{\mathbb{F}[\epsilon]}$ as fitting into an exact sequence $0 \rightarrow \mathfrak{M} \rightarrow \mathfrak{M}_{\mathbb{F}[\epsilon]} \rightarrow \mathfrak{M} \rightarrow 0$. If $\xi$ is an $\mathbb{F}[[u]]$-basis of $\mathfrak{M}$ (viewed as a row vector), then any $\mathfrak{S}_{\mathbb{F}}$-splitting corresponds to an $X \in$ $\operatorname{Mat}(\mathbb{F}((u)))$; the splitting sends $\xi$ onto $\xi(1+\epsilon X)$. As explained in 3.3.2, any such splitting gives rise to an $f \in\left(1 / u^{p}\right) \operatorname{Hom}(\mathfrak{M}, \mathfrak{M})$ such that

$$
\varphi_{\mathfrak{M}_{\mathbb{F}[\epsilon]}}=\left(\varphi_{\mathfrak{M}}+f \circ \varphi_{\mathfrak{M}}, \varphi_{\mathfrak{M}}\right) .
$$

Writing $\varphi_{\mathfrak{M}}(\xi)=\xi A$ for some matrix $A$, we compute that the matrix of $f$ with respect to $\xi$ is given by $A \varphi(X) A^{-1}-X$. As $\mathfrak{M}_{\mathbb{F}[\epsilon]}$ is strongly divisible, Proposition 3.3.5 implies that $X$ can be chosen so that $A \varphi(X) A^{-1}-X \in$ $\operatorname{Mat}(\mathbb{F}[[u]])$. Any such $\mathfrak{M}_{\mathbb{F}[\epsilon]}$ corresponds to a tangent vector, around the closed point corresponding to $\mathfrak{M}$, mapping onto the zero tangent vector at the closed point of Spec $R$. Such tangent vectors fit into the diagram

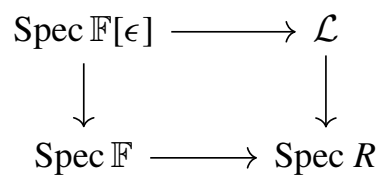

and so describe the tangent vectors of $\mathcal{L} \otimes_{R} \mathbb{F}$ at closed points. If every such tangent vector is zero, then it will follow that $\mathcal{L} \otimes_{R} \mathbb{F}$ is zero-dimensional and smooth, and so verifies the hypothesis of Proposition 5.3.2. In other words, to verify the hypothesis of Proposition 5.3.2, it suffices to show that $\mathfrak{M}_{\mathbb{F}[\epsilon]}=\mathfrak{M} \otimes_{\mathbb{F}}$ $\mathbb{F}[\epsilon]$. In other words, it suffices to show that if $X \in \operatorname{Mat}(\mathbb{F}((u)))$ is such that $A \varphi(X) A^{-1}-X \in \operatorname{Mat}(\mathbb{F}[[u]])$, then $X \in \operatorname{Mat}(\mathbb{F}[[u]])$.

We conclude with some examples in which Proposition 5.3.2 can be applied. 
EXAmPLE 5.3.4. Suppose $K=\mathbb{Q}_{p}$ and suppose $\mathbb{F}$ is sufficiently large. A $p$-adic Hodge type $\mathbf{v}$ concentrated in degree $[0, p]$ then corresponds to a pair of integers $0 \leqslant a \leqslant b \leqslant p$. Suppose $V_{\mathbb{F}}=\operatorname{Ind}_{G_{L}}^{G_{K}} W_{\mathbb{F}}$ is two-dimensional and irreducible, with $W_{\mathbb{F}}$ one-dimensional. In this case, we shall show $R_{\text {crys }}^{\mathbf{v}}$ is either zero or is formally smooth over $\mathbb{Z}_{p}$.

- First, using the discussion from 3.2.8, it is easy to verify that any $\mathfrak{M} \in \mathcal{L}$ crys $\left(V_{\mathbb{F}}\right)$ is of the form $f_{*} \mathfrak{N}$ for an $\mathfrak{N} \in \mathcal{L}^{\leqslant p}\left(W_{\mathbb{F}}\right)$. Of course, this is only true because $K=\mathbb{Q}_{p}$. Thus there exists an $\mathbb{F}[[u]]$-basis of $\mathfrak{M}$ with respect to which the matrix of $\varphi$ is given by $A=\left(\begin{array}{cc}0 & x u^{s} \\ x u^{r} & 0\end{array}\right)$ with $r, s \in[0, p]$ not equal and $x \in \mathbb{F}^{\times}$.

- Next, take $X=\left(\begin{array}{ll}a & b \\ c & d\end{array}\right)$ and compute

$$
A \varphi(X) A^{-1}-X=\left(\begin{array}{cc}
\varphi(d)-a & u^{s-r} \varphi(c)-b \\
u^{r-s} \varphi(b)-c & \varphi(a)-d
\end{array}\right)
$$

We want to verify the condition in 5.3.3, so assume $A \varphi(X) A^{-1}-X \in$ $\operatorname{Mat}(\mathbb{F}[[u]])$. It is easy to see this implies $a, d \in \mathbb{F}[[u]]$. For $c$ and $b$, we may assume that $r>s$; otherwise, interchange $c$ and $b$ in our argument. Let $x_{c}$ and $x_{b}$ denote the $u$-adic valuations of $c$ and $b$, respectively, and assume $x_{c}<0$. Then $r-s+p x_{b}=x_{c}$, and so $x_{b}<0$ also. This implies $s-r+p x_{c}=x_{b}$ and so $p\left(x_{b}+x_{c}\right)=\left(x_{c}+x_{b}\right)$, a contradiction. Now assume $x_{c} \geqslant 0$ and $x_{b}<0$. We still have $s-r+p x_{c}=x_{b}$ and so, as $s-r \geqslant-p$, we must have $x_{c}=0$. Thus $s-r=x_{b}$. On the other hand, we see that $r-s+p x_{b} \geqslant 0$, which is another contradiction. We conclude that $X \in \operatorname{Mat}(\mathbb{F}[[\mathrm{u}]])$, and so in this case $\mathcal{L} \otimes_{R} \mathbb{F}$ is zero-dimensional and reduced.

- Finally we argue that $\mathcal{L}^{\mathrm{v}}$ contains at most one closed point. One computes that for $\mathfrak{M}$ as above, $\mathcal{G}^{i}(\mathfrak{M})$ is zero unless $i=r$ or $s$, in which case it is onedimensional. This implies that the Breuil-Kisin modules associated with two closed points of $\mathcal{L}^{\mathbf{v}}$ must be abstractly isomorphic as Breuil-Kisin modules; they must therefore be equal since an abstract $\varphi$-equivariant isomorphism induces a $G_{K_{\infty}}$-equivariant automorphism of $V_{\mathbb{F}}$, and these are all given by scalar multiplication since $V_{\mathbb{F}}$ is irreducible.

EXAmple 5.3.5. Continue to assume that $K=\mathbb{Q}_{p}$ and that $\mathbb{F}$ is sufficiently large. We can then also treat the two-dimensional reducible case $V_{\mathbb{F}} \sim\left(\begin{array}{cc}\chi_{1} & c \\ 0 & \chi_{2}\end{array}\right)$, at least as long as $\chi_{1} \chi_{2}^{-1} \neq \chi_{\text {cyc }}$ so that $V_{\mathbb{F}}$ is cyclotomic-free. We can compute that $R_{\text {crys }}^{\mathbf{v}}$ is either zero or is formally smooth over $\mathbb{Z}_{p}$, except in the following exceptional cases: 
- When $\mathbf{v}=(a, a+p-1)$ and $V_{\mathbb{F}}$ is a split extension with $\chi_{1} \chi_{2}^{-1}$ equal to a nontrivial unramified character, $R_{\text {crys }}^{\mathbf{v}}$ is either zero or has two irreducible components, each of which is formally smooth.

- When $\mathbf{v}=(a, a+p-1), \chi_{1} \chi_{2}^{-1}=1$ and the cocycle $c(\sigma)$ is ramified, $R_{\text {crys }}^{\mathbf{v}}$ is either zero or formally smooth. If $c(\sigma)$ is unramified, then we are only able to deduce that $R^{\mathbf{v}}$ has a single irreducible component ([27] computes these rings directly in this case; they are not formally smooth; in fact, they are not even Cohen-Macaulay).

Let us only explain the claims in the last bullet point. We leave the rest as an exercise for the interested reader (the arguments are more straightforward). After twisting, we may suppose $V_{\mathbb{F}}$ admits an $\mathbb{F}$-basis $\xi_{\mathbb{F}}$ so that $\sigma\left(\xi_{\mathbb{F}}\right)=\xi_{\mathbb{F}}\left(\begin{array}{ccc}1 & c(\sigma) \\ 0 & 1\end{array}\right)$. Then the etale $\varphi$-module associated with $V_{\mathbb{F}}$, viewed as a submodule of $V_{\mathbb{F}} \otimes_{\mathbb{F}_{p}} C^{b}$, is generated by $\xi:=\xi_{\mathbb{F}}\left(\begin{array}{ll}1 & \alpha \\ 0 & 1\end{array}\right)$, where $\alpha \in C^{b}$ is such that $\sigma(\alpha)-\alpha=c(\sigma)$ for $\sigma \in G_{K_{\infty}}$.

- Note that $\varphi(\alpha)-\alpha \in \mathbb{F}((u))$. Note also that $\alpha$ is only well defined up to translation by elements of $\mathbb{F}((u))$. This allows us to assume that $\varphi(\alpha)-\alpha=\alpha_{0}+\alpha_{-1} u^{-1}+\cdots+\alpha_{-n} u^{-n}$ for some $\alpha_{i} \in \mathbb{F}$ and some $p>n \geqslant 0$. Let us choose $\alpha$ so that $n$ is minimal. If $\sigma \mapsto c(\sigma)$ is unramified, then we can clearly take $\alpha \in \bar{k} \otimes_{\mathbb{F}_{p}} \mathbb{F}$ and in this case $n=0$. Conversely, if $n=0$, then $\alpha \in \bar{k} \otimes_{\mathbb{F}_{p}} \mathbb{F}$ and so $\sigma \mapsto c(\sigma)$ is unramified.

- We first compute the set of $\mathfrak{M} \in \mathcal{L}^{\leqslant p}\left(V_{\mathbb{E}}\right)$. Any such $\mathfrak{M}$ is generated by $\xi B$ for some $B \in \mathrm{GL}_{2}(\mathbb{F}((u)))$. Using the Iwasawa decomposition for $\mathrm{GL}_{2}(\mathbb{F}((u)))$, we may assume $B=\left(\begin{array}{cc}u^{r} & b \\ 0 & u^{s}\end{array}\right)$ for some $b \in \mathbb{F}((u))$. With respect to $\xi B$, the matrix of $\varphi$ is given by

$$
A=\left(\begin{array}{cc}
u^{(p-1) r} & u^{-r}\left(\varphi(b)-u^{(p-1) s} b+u^{p s}(\varphi(\alpha)-\alpha)\right) \\
0 & u^{(p-1) s}
\end{array}\right) .
$$

Thus $r, s \in[0,1]$. When $r=s=0$, we must have $\varphi(b)-b+\varphi(\alpha)-\alpha \in \mathbb{F}[[u]]$, which is only possible if $\varphi(\alpha)-\alpha \in \mathbb{F}[[u]]$ and $b \in \mathbb{F}[[u]]$. Thus when $c(\sigma)$ is unramified, we obtain a single element of $\mathcal{L}^{\leqslant p}\left(V_{\mathbb{F}}\right)$, and otherwise this case contributes no elements. For $r=0, s=1$, we must have $\varphi(b)-u^{p-1} b+u^{p}(\varphi(\alpha)-\alpha) \in \mathbb{F}[[u]]$. This occurs if and only if $b \in \mathbb{F}[[u]]$, so in this case, we obtain a single element of $\mathcal{L} \leqslant p\left(V_{\mathbb{F}}\right)$. If $r=1, s=0$, then we must have $\varphi(b)-b+\varphi(\alpha)-\alpha \in u \mathbb{F}[[u]]$. We see this is only possible if $\varphi(\alpha)-\alpha=0$ (that is, $c(\sigma)=0$ ) and $b \in \mathbb{F}[[u]]$. In this case, we obtain multiple elements of $\mathcal{L}^{\leqslant p}\left(V_{\mathbb{F}}\right)$, one for every $b \in \mathbb{F}$. Finally, if $r=s=1$, then we must have $\varphi(b)-u^{p-1} b+u^{p}(\varphi(\alpha)-\alpha) \in u \mathbb{F}[[u]]$, and one sees that this case contributes one element to $\mathcal{L}^{\leqslant p}\left(V_{\mathbb{F}}\right)$. 
- We assert that each of the $\mathfrak{M} \in \mathcal{L} \leqslant p\left(V_{\mathbb{F}}\right)$ from the previous bullet point lies in $\mathcal{L}_{\text {crys }}^{\leqslant p}\left(V_{\mathbb{F}}\right)$. This can be done by first checking each is strongly divisible (which is easy to do by hand). Then use Lemma 5.1.6 to deduce each is contained in $\mathcal{L}_{\text {crys }}^{\leqslant p}\left(V_{\mathbb{F}}\right)$, where $V_{\mathbb{F}}$ is equipped with some $G_{K}$-action extending the $G_{K_{\infty}}$-action. Finally use that there is at most one way to extend the $G_{K_{\infty}}$ action on $V_{\mathbb{F}}$ to a $G_{K}$-action, because $V_{\mathbb{F}}$ is cyclotomic-free.

- Let us now focus on the case $\mathbf{v}=(0, p-1)$. We first suppose $c(\sigma)$ is nonzero. From the above, $\mathcal{L}^{\mathbf{v}}$ consists of one closed point $\mathfrak{M}$ admitting a basis on which $\varphi$ acts by $\left(\begin{array}{ll}1 & u^{p} \beta \\ 0 & u^{p-1}\end{array}\right)$, where $\beta=\varphi(\alpha)-\alpha$. To compute the tangent vectors of $\mathcal{L} \otimes_{R} \mathbb{F}$ around this point, take $X=\left(\begin{array}{ll}a & b \\ c & d\end{array}\right)$. Then $A \varphi(X) A^{-1}-X$ equals

$$
\left(\begin{array}{cc}
\varphi(a)-a+u^{p} \beta \varphi(c) & u^{1-p}\left(u^{p} \beta\left(\varphi(d)-\varphi(a)-u^{p} \beta \varphi(c)\right)+\varphi(b)-u^{p-1} b\right) \\
u^{p-1} \varphi(c)-c & -u^{p} \beta \varphi(c)+\varphi(d)-d
\end{array}\right) .
$$

Assume that $A \varphi(X) A^{-1}-X \in \operatorname{Mat}(\mathbb{F}[[u]])$. From $u^{p-1} \varphi(c)-c \in \mathbb{F}[[u]]$, we deduce $c$ has $u$-adic valuation $\geqslant-1$. If this valuation is -1 , then $\varphi(a)-a+u^{p} \beta \varphi(c) \in \mathbb{F}[[u]]$ implies $v(\varphi(a)-a)=v(\beta)$. By construction, $p<v(\beta) \leqslant 0$ while $v(\varphi(a)-a)=p v(a)$ unless $a \in \mathbb{F}[[u]]$, so we must have $a, \beta \in \mathbb{F}[[u]]$. Thus $c \notin \mathbb{F}[[u]]$ implies $c(\sigma)$ is unramified. Regardless of the valuation of $c$, we see $\alpha \in \mathbb{F}[[u]]$. Similarly $d \in \mathbb{F}[[u]]$, and also $b \in \mathbb{F}[[u]]$. We conclude that if $c(\sigma) \neq 0$, then $\mathcal{L}^{\mathbf{v}} \otimes_{R} \mathbb{F}$ is a reduced point when $c(\sigma)$ is ramified and a nonreduced point if $c(\sigma)$ is unramified.

- Finally we consider when $c(\sigma)=0$. In this case, we may construct a morphism $\mathbb{A}_{\mathbb{F}}^{1} \rightarrow \mathcal{L}^{\mathbf{v}}$ given by the element $\mathfrak{M}_{\mathbb{F}[T]} \in \mathcal{L}_{\text {crys }}^{\leqslant p}\left(V_{\mathbb{F}} \otimes_{\mathbb{F}} \mathbb{F}[T]\right)$ with basis

$$
\xi\left(\begin{array}{ll}
u & T \\
0 & 1
\end{array}\right)
$$

for $\xi$ as above. Since $\mathcal{L}^{\mathbf{v}}$ is projective, this morphism extends to a morphism $\mathbb{P}_{\mathbb{F}}^{1} \rightarrow \mathcal{L}^{\mathbf{v}}$, which is an isomorphism.

These calculations recover those of [23, 1.7.14]; see also [27].

\section{Acknowledgements}

I would like to thank Frank Calegari, Mark Kisin, Tong Liu and James Newton for helpful conversations and correspondence. Special thanks also go to the anonymous referee for making a number of helpful suggestions and comments. This work was done at the Max Planck Institute for Mathematics in Bonn, and it is a pleasure to thank this institution for their support. 


\section{Conflict of Interest: None.}

\section{References}

[1] T. Barnet-Lamb, T. Gee, D. Geraghty and R. Taylor, 'Potential automorphy and change of weight', Ann. of Math. (2) 179(2) (2014), 501-609.

[2] R. Bartlett, 'Inertial and Hodge-Tate weights of crystalline representations', Math. Ann. 376 (2020), 645-681.

[3] R. Bartlett, 'Potentially diagonalisable lifts with controlled Hodge-Tate weights' Preprint, 2018, arXiv:1812.02042.

[4] R. Bartlett, 'Potential diagonalisability of pseudo-Barsotti-Tate representations', Preprint, 2020, arXiv:2001.08660.

[5] B. Bhatt, M. Morrow and P. Scholze, 'Integral p-adic Hodge theory', Publ. Math. Inst. Hautes Études Sci. 128 (2018), 219-397.

[6] N. Bourbaki, Éléments de mathématique. Fascicule XXVIII. Algèbre commutative. Chapitre 3: Graduations, filtrations et topologies. Chapitre 4: Idéaux premiers associés et décomposition primaire. Actualités Scientifiques et Industrielles, No. 1293. Hermann, Paris, 1961.

[7] C. Breuil and A. Mézard, 'Multiplicités modulaires et représentations de $\mathrm{GL}_{2}\left(\mathbf{Z}_{p}\right)$ et de $\operatorname{Gal}\left(\overline{\mathbf{Q}_{p}} / \mathbf{Q}_{p}\right)$ en $l=p$ ', Duke Math. J. 115(2) (2002), 205-310. With an appendix by Guy Henniart.

[8] P. Colmez, 'Théorie d'Iwasawa des représentations de de Rham d'un corps local', Ann. of Math. (2) 148(2) (1998), 485-571.

[9] J.-M. Fontaine, 'Représentations $p$-adiques des corps locaux. I', in The Grothendieck Festschrift, Vol. II, Progress in Mathematics, 87 (Birkhäuser Boston, Boston, MA, 1990), 249-309.

[10] J.-M. Fontaine, 'Le corps des périodes p-adiques', Astérisque 223 (1994), 59-111. With an appendix by Pierre Colmez, Périodes $p$-adiques (Bures-sur-Yvette, 1988).

[11] H. Gao, 'Crystalline liftings and weight part of Serre's conjecture', Israel J. Math 221(1) (2017), 117-164.

[12] H. Gao, 'Breuil-Kisin modules and integral p-adic Hodge theory', Preprint, 2019, arXiv:19 05.08555 .

[13] H. Gao and T. Liu, 'A note on potential diagonalizability of crystalline representations', Math. Ann. 360(1-2) (2014), 481-487.

[14] T. Gee, 'A modularity lifting theorem for weight two Hilbert modular forms', Math. Res. Lett. 13(5-6) (2006), 805-811.

[15] T. Gee and M. Kisin, 'The Breuil-Mézard conjecture for potentially Barsotti-Tate representations', Forum Math. Pi 2, e1 (2014), 56.

[16] T. Gee, T. Liu and D. Savitt, 'The Buzzard-Diamond-Jarvis conjecture for unitary groups', J. Amer. Math. Soc. 27(2) (2014), 389-435.

[17] T. Gee, T. Liu and D. Savitt, 'The weight part of Serre's conjecture for GL(2)', Forum Math. Pi 3 e2 (2015).

[18] A. Grothendieck, 'Éléments de géométrie algébrique. III. Étude cohomologique des faisceaux cohérents. I', Inst. Hautes Études Sci. Publ. Math. 11 (1961), 167.

[19] A. Grothendieck, 'Éléments de géométrie algébrique. IV. Étude locale des schémas et des morphismes de schémas IV', Inst. Hautes Études Sci. Publ. Math. 32 (1967), 361.

[20] M. Kisin, 'Crystalline representations and F-crystals', in Algebraic Geometry and Number Theory, Progress in Mathematics, 253 (Birkhäuser Boston, Boston, MA, 2006), 459-496. 
[21] M. Kisin, 'Potentially semi-stable deformation rings', J. Amer. Math. Soc. 21(2) (2008), 513-546.

[22] M. Kisin, 'Moduli of finite flat group schemes, and modularity', Ann. of Math. (2) 170(3) (2009), 1085-1180.

[23] M. Kisin, 'The Fontaine-Mazur conjecture for $\mathrm{GL}_{2}$ ', J. Amer. Math. Soc. 22(3) (2009), 641-690.

[24] M. Kisin, 'Integral models for Shimura varieties of abelian type', J. Amer. Math. Soc. 23(4) (2010), 967-1012.

[25] T. Liu, 'The correspondence between Barsotti-Tate groups and Kisin modules when $p=2$ ', J. Théor. Nombres Bordeaux 25(3) (2013), 661-676.

[26] Y. Ozeki, 'Full faithfulness theorem for torsion crystalline representations', New York J. Math. 20 (2014), 1043-1061.

[27] F. Sander, 'Hilbert-Samuel multiplicities of certain deformation rings', Math. Res. Lett. 21(3) (2014), 605-615.

[28] The Stacks Project Authors. Stacks Project. http://stacks.math.columbia.edu, 2017.

[29] X. Wang, 'Weight elimination in two dimensions when $p=2$ ', Preprint, 2017, arXiv:1711. 09035. 